\title{
CRYSTAL BASES AND TWO-SIDED CELLS OF QUANTUM AFFINE ALGEBRAS
}

\author{
JONATHAN BECK AND HIRAKU NAKAJIMA
}

\section{INTRODUCTION}

Let $\mathfrak{g}$ be an affine Kac-Moody Lie algebra. Let $\mathbf{U}=\mathbf{U}(\mathfrak{g})$ be its quantum enveloping algebra introduced by Drinfeld and Jimbo, and let $\mathbf{U}^{+}$be its positive part. The purpose of this paper is twofold. First, we define a basis $B$ of $\mathbf{U}^{+}$which is an analog of a PBW basis of $\mathbf{U}$ for a finite dimensional simple Lie algebra. It has the following properties (see Theorem 3.13):

(1) Each element of $B$ is a product of a monomial in 'real root vectors' and a Schur function in 'imaginary root vectors'.

(2) The transition matrix between $B$ and Kashiwara-Lusztig's global crystal basis (or canonical basis) $G(\mathcal{B}(-\infty))$ is upper-triangular with 1's on the diagonal (with respect to a certain explicitly defined ordering) and with above diagonal entries in $q_{s}^{-1} \mathbf{Z}\left[q_{s}^{-1}\right]$.

When $\mathfrak{g}$ is symmetric or of type $A_{2}^{(2)}$, our basis coincides with the one constructed by Beck-Chari-Pressley [6] or Akasaka [1] respectively, where a property weaker than (2) was established.

Second, we study the global crystal basis $\mathcal{B}(\widetilde{\mathbf{U}})$ of the modified quantum enveloping algebra $\widetilde{\mathbf{U}}$ defined by Lusztig 22 . We obtain a Peter-Weyl like decomposition of the crystal $\mathcal{B}(\widetilde{\mathbf{U}})$ (Theorem 4.18), as well as an explicit description of two-sided cells of $\mathcal{B}(\widetilde{\mathbf{U}})$ and the limit algebra of $\widetilde{\mathbf{U}}$ at $q=0$ (Theorem 6.44). These results had been conjectured by Nakashima [31], Kashiwara [18] and Lusztig [24] respectively. For type $A_{1}^{(1)}$, the former was proved in [31].

Our results are based on the study of "extremal weight modules" $V(\lambda)$ for $\lambda \in P$, introduced by Kashiwara [17. These $\widetilde{\mathbf{U}}$-modules have global crystal bases $G(\mathcal{B}(\lambda))$, and for $\lambda \in \bigcup_{w \in W} w\left(P_{+}\right)$(the Tits cone) are isomorphic to irreducible highest weight modules [loc. cit.]. Outside of the Tits cone, or equivalently in the affine case for level zero weights, the structure of these modules has been studied by AkasakaKashiwara [2] and Kashiwara [18. In particular, Kashiwara made conjectures on the crystal $\mathcal{B}(\lambda)[18, \S 13]$. The present authors independently proved his conjectures for symmetric $\mathfrak{g}[5,[30]$. Both proofs used [6]. (The relevance of [6] to his conjectures was already pointed out in [18].) In this paper, we first generalize [6] to the nonsymmetric cases modulo sign and then proceed to prove the conjectures on $\mathcal{B}(\lambda)$ modulo sign. Next we remove the sign ambiguity. Finally, the above mentioned properties of $\mathcal{B}(\widetilde{\mathbf{U}})$ are established.

2000 Mathematics Subject Classification. 17B37.

Key words and phrases. crystal base, quantum affine algebra.

The work of H.N. is supported by the Grant-in-aid for Scientific Research (No.13640019), JSPS. 
The construction of the basis $B$ is similar to the previous construction [6, 1], although there are two new ideas worth remarking on. First, we use the level zero extremal weight modules $V(\lambda)$ in an essential way to check that after specializing at $q=\infty$ our basis elements equal canonical basis elements (rather than equal up to sign, which is easier and is also proved here). Note that in [6] the sign check depends on a positivity result of Lusztig which is available only for the symmetric case. Second, we obtain the fact that our basis $B$ is an integral basis (i.e., a basis of the Lusztig $\mathbf{Z}\left[q_{s}, q_{s}^{-1}\right]$-form of $\mathbf{U}^{+}$) in an interesting way. This result follows from the upper triangularity (2) above and uses the canonical basis and the extremal weight modules in an essential way. Our proof is quite different from the proofs 1. 6. where the result is obtained by explicitly checking commutation relations between root vectors.

Furthermore, it should be mentioned that the integrality property of $B$ also gives (when specializing $q=1$ ) a construction of an easily expressed basis of the $\mathbf{Z}$-form of the universal enveloping algebra of $\mathfrak{g}$. Such a basis has been constructed [14, 28], but these results rely on directly examining all possible commutation relations between elements of the monomials forming the basis. Here we obtain this result as a corollary to the existence of the canonical basis of $\mathbf{U}^{+}$.

Let us make one more remark regarding $B$. Recall that Lusztig used a PBW basis to give an alternative definition of the canonical basis for finite type $\mathfrak{g}$ (an 'elementary algebraic definition') 21]. Namely, the canonical basis is characterized as 1) an integral basis, 2) invariant under the bar involution, and 3) upper triangular with respect to the PBW basis. The existence of such a basis is guaranteed by the upper triangular property of the bar involution with respect to the PBW basis. Our definition of elements of $B$ is completely elementary and we can prove the upper triangular property without using the global crystal basis. For symmetric or type $A_{2}^{(2)}$, [1], 6] proved that $B$ is a basis of the integral form of $\mathbf{U}^{+}$. The same argument as in the finite case then gives us the 'elementary algebraic definition' of the global crystal basis. (See Theorem 3.45.) However, when $B$ is not a priori known to be an integral basis (i.e., not in the symmetric or $A_{2}^{(2)}$ case), we only show the matrix expressing the bar involution has entries in $\mathbf{Q}\left(q_{s}\right)$, and we do not give an alternative algebraic definition of $G(\mathcal{B}(-\infty))$. We hope that we might avoid the integrality requirement completely in the near future. In any case, our basis $B$ gives a parametrization of the global crystal basis and serves us here to prove the above mentioned conjectures on $\mathcal{B}(\widetilde{\mathbf{U}})$.

We review the organization of this paper in detail. In $\S 2$ we introduce notation and preliminary results from [18], [30] and [5]. Next, in $\S 3$ we construct the basis $B$ for the integral form ${ }_{\mathcal{A}} \mathbf{U}^{+}$with the properties described above (up to sign). In $\S 4$ we consider the crystal structure of $\widetilde{\mathbf{U}}$ in more detail. We verify the conjectures of [18, §13] (up to sign) which describe the the crystal structure $\mathcal{B}(\lambda)$ of $V(\lambda)$. $\mathcal{B}(\lambda)$ decomposes into a product of $\mathcal{B}_{W}(\lambda) \times \operatorname{Irr} G_{\lambda}$, where for $\lambda=\sum_{i} \lambda_{i} \varpi_{i}, \mathcal{B}_{W}(\lambda)$ denotes the crystal of $W(\lambda)=\bigotimes_{i} W\left(\varpi_{i}\right)^{\otimes \lambda_{i}}$ and $\operatorname{Irr} G_{\lambda}$ denotes irreducible representations of $G_{\lambda}=\prod_{i} G L_{\lambda_{i}}(\mathbf{C})$. This decomposition is then used to give a $\mathfrak{g} \times \mathfrak{g}$ bicrystal decomposition of $\mathcal{B}(\widetilde{\mathbf{U}}) \cong \bigsqcup_{\lambda \in P} \mathcal{B}_{0}(\lambda) \times \mathcal{B}(\lambda) / \hat{W}$, where $\mathcal{B}_{0}(\lambda)$ denotes the connected component of $\mathcal{B}(\lambda)$ containing the extremal weight vector $v_{\lambda}$ and $\hat{W}$ is the affine Weyl group. In $\S 5$ we pause to remove the sign ambiguity in $\S 3$ and $\S 4$. 
In $\S 6$ we study the global basis of the level zero modified quantum affine algebra $\widetilde{\mathbf{U}}=\bigoplus_{\lambda \in P_{\mathrm{cl}}^{0}} \mathbf{U} a_{\lambda}$. To each $\lambda \in P_{\mathrm{cl},+}^{0}$ we associate a two sided ideal which is the intersection of the annihilators of all $V\left(\lambda^{\prime}\right)$ for $\lambda^{\prime}$ outside the cone $\lambda+P_{\mathrm{cl},+}^{0}$ modulo this same ideal further intersected with the annihilator of $V(\lambda)$. We show that these ideals have crystal bases $\tilde{\mathcal{B}}[\lambda]$ which have globalizations which partition the global basis of $\widetilde{\mathbf{U}}$. We use this partition to describe the cell structure of $\mathcal{B}(\widetilde{\mathbf{U}})$ and to verify the conjectures which appear in 24.

Lusztig's conjectures on two-sided cells were based on his conjectures [20] on cells of an affine Hecke algebra, which as far as the authors know, are still open. In 20] Lusztig made a deep connection between two-sided cells of the affine Hecke algebra and the geometry of Springer fibers. Our proof is based on extremal weight modules and is purely algebraic. However geometry is in the background, since extremal weight modules are isomorphic [30] to universal standard modules, which are defined as $K$-homology groups of certain quiver varieties introduced by the second author [29]. For example, values of the $a$-function introduced in $\S 6$ are equal to the dimensions of the quiver varieties, where the corresponding result for the affine Hecke algebra was proved in [20]. It is also worthwhile mentioning that the appearance of $G_{\lambda}$ is quite natural from quiver varieties.

While the authors were preparing this paper, K. McGerty posted an article [27] to the q-algebra archive where he proves Lusztig's conjecture for type $A_{n}^{(1)}$. His proof is completely different from our proof.

Acknowledgment. The authors are grateful to Ilaria Damiani who sent us a reprint of 12].

\section{Preliminaries}

2.1. Affine Kac-Moody Lie algebras. We fix a realization $\mathfrak{g}=\mathfrak{g}\left(X_{N}^{(r)}\right)$. Here $X_{N}^{(r)}$ is a diagram from Table Aff $r$ of [15, Section 4.8], except in the case of $X_{N}^{(r)}=A_{2 n}^{(2)}(n \geq 1)$, where we reverse the numbering of the simple roots. Let its Cartan subalgebra be $\mathfrak{h}$. We denote by $I$ the index set of simple roots. The numbering gives us an identification $I=\{0,1, \ldots, n\}$. Let $\left\{\alpha_{i}\right\}_{i \in I} \subset \mathfrak{h}$ (resp. $\left\{h_{i}\right\}_{i \in I} \subset \mathfrak{h}^{*}$ ) denote the set of simple roots (resp. simple coroots), where $\left\langle h_{i}, \alpha_{j}\right\rangle=$ $a_{i j}$, where $a_{i j}$ is the Cartan matrix of $\mathfrak{g}$. Fix $d$ so that $\left\langle d, \alpha_{j}\right\rangle=\delta_{0 j}$. Denote by $P^{*}=\bigoplus_{i \in I} \mathbf{Z} h_{i} \oplus \mathbf{Z} d$ the dual weight lattice and by $P=\operatorname{Hom}_{\mathbf{Z}}\left(P^{*}, \mathbf{Z}\right)$ the weight lattice. Let $Q=\sum_{i \in I} \mathbf{Z} \alpha_{i} \subset P$ denote the root lattice, $\Delta$ the root system and $\Delta^{\mathrm{re}}=\Delta \backslash \mathbf{Z} \delta$ the set of real roots. Fix the fundamental weights $\Lambda_{i} \in P$ defined by $\left\langle h_{i}, \Lambda_{j}\right\rangle=\delta_{i j},\left\langle d, \Lambda_{j}\right\rangle=0$. Denote by $Q_{+}$the semigroup generated by positive roots $\sum_{i \in I} \mathbf{Z}_{\geq 0} \alpha_{i} ; P_{+}=\left\{\lambda \in P \mid\left\langle h_{i}, \lambda\right\rangle \geq 0\right.$ for all $\left.i \in I\right\}$ the semigroup of integral dominant weights. Let $\Delta^{ \pm}=\Delta \cap\left( \pm Q_{+}\right)$be the set of positive and negative roots respectively.

The center of $\mathfrak{g}$ is 1 -dimensional and is spanned by $c=\sum_{i \in I} a_{i}^{\vee} h_{i}$, where $a_{i}^{\vee}$ are the labels of the dual diagram to $X_{N}^{(r)}$. $c$ is characterized as the positive combination of $h_{i}, i \in I$, for which $\left\{h \in P^{*} \mid\left\langle h, \alpha_{j}\right\rangle=0\right.$ for all $\left.j \in I\right\}=\mathbf{Z} c$. Let $\delta$ be the unique element $\delta=\sum_{i \in I} a_{i} \alpha_{i}\left(a_{i} \in \mathbf{Z}_{\geq 0}\right.$, where $a_{i}$ are the numerical labels of $X_{N}^{(r)}$, and give a linear dependence between the columns of $\left.a_{i j}\right)$ satisfying $\left\{\lambda \in Q \mid\left\langle h_{i}, \lambda\right\rangle=\right.$ 0 for all $i \in I\}=\mathbf{Z} \delta$. We denote by $h$ the Coxeter number $\sum_{i \in I} a_{i}$ and by $h^{\vee}$ the dual Coxeter number $\sum_{i \in I} a_{i}^{\vee}$. 
Denote the affine Weyl group by $\hat{W} \subset O\left(\mathfrak{h}^{*}\right)\left(=\right.$ the orthogonal group of $\mathfrak{h}^{*}$ with respect to $()$,$) generated by the simple reflections s_{i}(\lambda)=\lambda-\left\langle h_{i}, \lambda\right\rangle \alpha_{i}, \lambda \in \mathfrak{h}, i \in I$. Note that $w(\delta)=\delta$ for $w \in \hat{W}$. Denote by $($,$) the non-degenerate symmetric$ bilinear form on $\mathfrak{h}^{*}$ invariant under the Weyl group action, uniquely characterized by $\langle c, \lambda\rangle=(\delta, \lambda)$, for $\lambda \in \mathfrak{h}^{*}$. Note that $\left(\alpha_{i}, \alpha_{j}\right)=a_{i}^{\vee} a_{i}^{-1} a_{i j}$ for $i, j \in I$.

Let cl: $\mathfrak{h}^{*} \rightarrow \mathfrak{h}^{*} / \mathbf{Q} \delta$ be the canonical projection. Let $\mathfrak{h}^{* 0}=\left\{\lambda \in \mathfrak{h}^{*} \mid\langle c, \lambda\rangle=0\right\}$, and define the level zero weight lattice to be $P^{0}=P \cap \mathfrak{h}^{* 0}$. Let $\mathfrak{h}_{\mathrm{cl}}^{* 0}=\operatorname{cl}\left(\mathfrak{h}^{* 0}\right)$ and $P_{\mathrm{cl}}^{0}=\operatorname{cl}\left(P^{0}\right)$. Denote $\Delta_{\mathrm{cl}}=\operatorname{cl}\left(\Delta^{\mathrm{re}}\right)$. Since $w(\delta)=\delta$ and $\langle c, \lambda\rangle=(\delta, \lambda)$, the image of $\hat{W} \subset O\left(\mathfrak{h}^{* 0}\right)$ in $O\left(\mathfrak{h}_{\mathrm{cl}}^{* 0}\right)$ is well-defined and denoted $W_{\mathrm{cl}}$. Then $W_{\mathrm{cl}}$ is the Weyl group of the root system $\left(\Delta_{\mathrm{cl}}, \mathfrak{h}_{\mathrm{cl}}^{* 0}\right)$, which is reduced, except in type $A_{2 n}^{(2)}$ where it is of type $B C_{n}$. The bilinear form $($,$) on \mathfrak{h}^{*}$ descends to a bilinear form on $\mathfrak{h}_{\mathrm{cl}}^{* 0}$, which is denoted also by $($,$) . It is nondegenerate.$

We fix $0 \in I$ so that $W_{\mathrm{cl}}$ is generated by $\left\{s_{i} ; i \in I_{0}\right\}$, where $I_{0}=I \backslash\{0\}=$ $\{1,2, \ldots, n\}$. If $\mathfrak{g}$ is not of type $A_{2 n}^{(2)}$, the choice of 0 is unique up to a Dynkin diagram automorphism. In the case of $A_{2 n}^{(2)}$, there are two choices of 0 (either of the two extremal vertices of the Dynkin diagram), and $\left(\alpha_{0}, \alpha_{0}\right)=1$ or 4 , and accordingly $a_{0}=2$ or $1, a_{0}^{\vee}=1$ or 2 . Our choice of 0 is such that $\left(\alpha_{0}, \alpha_{0}\right)=4$. As mentioned above, this is opposite the numbering convention in 15 , but is natural when constructing $\mathfrak{g}=\mathfrak{g}\left(A_{2 n}^{(2)}\right)$ as a (twisted) loop algebra. We take $\left\{\operatorname{cl}\left(\alpha_{i}\right) \mid i \in I_{0}\right\}$ as a set of simple roots of $\Delta_{\mathrm{cl}}$, and the corresponding set $\Delta_{\mathrm{cl}}^{+}$of positive roots.

Let $\alpha^{\vee}=2 \alpha /(\alpha, \alpha)$. Let $Q^{\vee}=\sum_{\alpha \in \Delta^{\mathrm{re}}} \mathbf{Z}^{\vee}$. We set $Q_{\mathrm{cl}}=\operatorname{cl}(Q), Q_{\mathrm{cl}}^{\vee}=\operatorname{cl}\left(Q^{\vee}\right)$, $\widetilde{Q}=Q_{\mathrm{cl}} \cap Q_{\mathrm{cl}}^{\vee}$. We have an exact sequence

$$
1 \longrightarrow \widetilde{Q} \stackrel{t}{\longrightarrow} \hat{W} \stackrel{\mathrm{cl}}{\longrightarrow} W_{\mathrm{cl}} \longrightarrow 1,
$$

where $t$ is the translation operator given by 15 (6.5.2)], and cl is the above projection $\hat{W} \rightarrow W_{\mathrm{cl}}$. By abuse of notation we denote $t(\xi)$ simply by $\xi$. For any $\alpha \in \Delta^{\text {re }}$, let $\tilde{\alpha}$ be the element in $\widetilde{Q} \cap \mathbf{Q}_{>0} \operatorname{cl}(\alpha)$ with the smallest length. We set

$$
\tilde{\Delta}=\left\{\tilde{\alpha} ; \alpha \in \Delta^{\mathrm{re}}\right\} .
$$

Then $\tilde{\Delta}$ is a reduced root system, and $\widetilde{Q}$ is the root lattice of $\tilde{\Delta}$.

An affine Lie algebra $\mathfrak{g}$ is either untwisted or the dual of an untwisted affine Lie algebra or $A_{2 n}^{(2)}$ :

(i) If $\mathfrak{g}$ is untwisted, then $2 /(\alpha, \alpha) \in \mathbf{Z}, \widetilde{Q}=Q_{\mathrm{cl}}^{\vee} \subset Q_{\mathrm{cl}}, \tilde{\alpha}=\operatorname{cl}\left(\alpha^{\vee}\right), \tilde{\Delta}=$ $\operatorname{cl}\left(\Delta^{\mathrm{re} \vee}\right)$.

(ii) If $\mathfrak{g}$ is the dual of an untwisted algebra, then $(\alpha, \alpha) / 2 \in \mathbf{Z}, \widetilde{Q}=Q_{\mathrm{cl}} \subset Q_{\mathrm{cl}}^{\vee}$, $\tilde{\alpha}=\operatorname{cl}(\alpha), \tilde{\Delta}=\operatorname{cl}\left(\Delta^{\mathrm{re}}\right)$.

(iii) If $\mathfrak{g}=\mathfrak{g}\left(A_{2 n}^{(2)}\right)$, then $(\alpha, \alpha) / 2=1 / 2,1$, or $2, \widetilde{Q}=Q_{\mathrm{cl}}=Q_{\mathrm{cl}}^{\vee}$, and

$$
\tilde{\alpha}= \begin{cases}\operatorname{cl}(\alpha) & \text { if }(\alpha, \alpha) \neq 4 \\ \operatorname{cl}(\alpha) / 2 & \text { if }(\alpha, \alpha)=4\end{cases}
$$

Note that $(\delta-\alpha) / 2 \in \Delta^{\mathrm{re}}$ if $(\alpha, \alpha)=4 . \tilde{\Delta}$ is of type $B_{n}$.

For $\alpha \in \Delta^{\text {re }}$ or $\alpha \in \Delta_{\text {cl }}$, we set

$$
d_{\alpha}=\max \left(1, \frac{(\alpha, \alpha)}{2}\right),
$$


and $d_{i}=d_{\alpha_{i}}$. We have $m \delta+\alpha \in \Delta^{\mathrm{re}} \Longleftrightarrow d_{\alpha} \mid m$. If $X_{N}^{(r)} \neq A_{2 n}^{(2)}$, then $\tilde{\alpha}=$ $d_{\alpha} \operatorname{cl}\left(\alpha^{\vee}\right)$.

Let $P_{\mathrm{cl}}^{0}\left(\right.$ resp. $\left.P_{\mathrm{cl}}^{0 \vee}\right)$ be the dual of $Q_{\mathrm{cl}}^{\vee}\left(\right.$ resp. $\left.Q_{\mathrm{cl}}\right)$, considered as a lattice of $\mathfrak{h}_{\mathrm{cl}}^{0 *}$ via (, ). Set $\widetilde{P}=P_{\mathrm{cl}}^{0} \cap P_{\mathrm{cl}}^{0 \vee}=\left(Q_{\mathrm{cl}}+Q_{\mathrm{cl}}^{\vee}\right)^{*}$. The sets $\Delta, \Delta^{\vee}$ are invariant under the translation by an element of $\widetilde{P}$. We define the extended affine Weyl group by $\widetilde{W}=\widetilde{P} \rtimes W_{\text {cl. }}$. Let $\mathcal{T}=\left\{w \in \widetilde{W} \mid w\left(\Delta^{+}\right) \subset \Delta^{+}\right\}$. It is a subgroup of the group of Dynkin diagram automorphisms. We have $\widetilde{W}=\mathcal{T} \ltimes \hat{W}$. The length function $\ell: \hat{W} \rightarrow \mathbf{N}$ extends to $\ell: \widetilde{W} \rightarrow \mathbf{N}$ where $\ell(\tau w)=\ell(w)$ for $\tau \in \mathcal{T}, w \in \hat{W}$.

Let us denote by $\omega_{i}^{\vee}\left(i \in I_{0}\right)$ the fundamental coweights of the root system $\left(\Delta_{\mathrm{cl}}, \mathfrak{h}_{\mathrm{cl}}^{* 0}\right)$, i.e., $\left(\operatorname{cl}\left(\alpha_{i}\right), \omega_{j}^{\vee}\right)=\delta_{i j}$ for $i, j \in I_{0}$. Let $\tilde{\omega}_{i}=d_{i} \omega_{i}^{\vee}$. Then $\left\{\tilde{\omega}_{i}\right\}_{i \in I_{0}}$ is a basis of $\widetilde{P}$. We have

$$
\begin{aligned}
s_{j} \tilde{\omega}_{i} & =\tilde{\omega}_{i} s_{j} \quad(i \neq j), \\
s_{i} \tilde{\omega}_{i} s_{i} \tilde{\omega}_{i}^{-1}=t\left(-d_{i} \alpha_{i}^{\vee}\right) & = \begin{cases}\tilde{\alpha}_{i}^{-1} & \text { if }\left(X_{N}^{(r)}, i\right) \neq\left(A_{2 n}^{(2)}, n\right), \\
\tilde{\alpha}_{i}^{-2} & \text { if }\left(X_{N}^{(r)}, i\right)=\left(A_{2 n}^{(2)}, n\right) .\end{cases}
\end{aligned}
$$

Set

$$
\begin{gathered}
\mathcal{R}_{>}=\left\{\alpha \in \Delta^{+} \mid \operatorname{cl}(\alpha) \in \Delta_{\mathrm{cl}}^{+}\right\}, \quad \mathcal{R}_{<}=\left\{\alpha \in \Delta^{+} \mid \operatorname{cl}(\alpha) \in-\Delta_{\mathrm{cl}}^{+}\right\}, \\
\mathcal{R}_{0}=\left\{(m \delta, i) \in \mathbf{Z} \delta \times I_{0}\left|m>0, d_{i}\right| m\right\}, \\
\mathcal{R}=\mathcal{R}_{>} \sqcup \mathcal{R}_{0} \sqcup \mathcal{R}_{<} .
\end{gathered}
$$

These are sets of positive roots counted with multiplicities.

2.2. Quantum affine algebras. We define the quantum affine algebra $\mathbf{U}=\mathbf{U}(\mathfrak{g})$ following the normalization in [2, 18]. Let $q$ be an indeterminate. For nonnegative integers $n \geq r$, define

$$
[n]_{q}=\frac{q^{n}-q^{-n}}{q-q^{-1}}, \quad[n]_{q} != \begin{cases}{[n]_{q}[n-1]_{q} !} & (n>0), \\
1 & (n=0), \quad\left[\begin{array}{l}
n \\
r
\end{array}\right]_{q}=\frac{[n]_{q} !}{[r]_{q} ![n-r]_{q} !} .\end{cases}
$$

We fix the smallest positive integer $d$ such that $d\left(\alpha_{i}, \alpha_{i}\right) / 2 \in \mathbf{Z}$ for any $i \in I$. We set $q_{s}=q^{1 / d}$.

Define the quantum affine algebra $\mathbf{U}$ to be the associative algebra with 1 over $\mathbf{Q}\left(q_{s}\right)$ generated by elements $E_{i}, F_{i}(i \in I), q^{h}\left(h \in d^{-1} P^{*}\right)$, with defining relations.

$$
\begin{gathered}
q^{0}=1, \quad q^{h} q^{h^{\prime}}=q^{h+h^{\prime}}, \\
q^{h} E_{i} q^{-h}=q^{\left\langle h, \alpha_{i}\right\rangle} E_{i}, \quad q^{h} F_{i} q^{-h}=q^{-\left\langle h, \alpha_{i}\right\rangle} F_{i}, \\
E_{i} F_{j}-F_{j} E_{i}=\delta_{i j} \frac{t_{i}-t_{i}^{-1}}{q_{i}-q_{i}^{-1}}, \\
\sum_{p=0}^{b}(-1)^{p} E_{i}^{(p)} E_{j} E_{i}^{(b-p)}=\sum_{p=0}^{b}(-1)^{p} F_{i}^{(p)} F_{j} F_{i}^{(b-p)}=0 \quad \text { for } i \neq j,
\end{gathered}
$$

where $q_{i}=q^{\left(\alpha_{i}, \alpha_{i}\right) / 2}, t_{i}=q^{\left(\alpha_{i}, \alpha_{i}\right) h_{i} / 2}, b=1-\left\langle h_{i}, \alpha_{j}\right\rangle, E_{i}^{(p)}=E_{i}^{p} /[p] q_{i} !, F_{i}^{(p)}=$ $F_{i}^{p} /[p]_{q_{i}} !$.

Let $\mathbf{U}^{\prime}$ be the quantized enveloping algebra with $P_{\mathrm{cl}}=\mathrm{cl}(P)$ as a weight lattice. It is the subalgebra of $\mathbf{U}$ generated by $E_{i}, F_{i}(i \in I), q^{h}\left(h \in d^{-1} \bigoplus_{i} \mathbf{Z} h_{i}\right)$. 
Let $\mathbf{U}^{+}$(resp. $\mathbf{U}^{-}$) be the $\mathbf{Q}\left(q_{s}\right)$-subalgebra of $\mathbf{U}$ generated by elements $E_{i}$ 's (resp. $F_{i}$ 's). Let $\mathbf{U}^{0}$ be the $\mathbf{Q}\left(q_{s}\right)$-subalgebra generated by elements $q^{h}\left(h \in d^{-1} P^{*}\right)$. We have the triangular decomposition $\mathbf{U} \cong \mathbf{U}^{+} \otimes \mathbf{U}^{0} \otimes \mathbf{U}^{-}$.

For $\xi \in Q$, we define the root space $\mathbf{U}_{\xi}$ by

$$
\mathbf{U}_{\xi}=\left\{x \in \mathbf{U} \mid q^{h} x q^{-h}=q^{\langle h, \xi\rangle} x \text { for all } h \in P^{*}\right\} .
$$

Let $\mathcal{A}=\mathbf{Z}\left[q_{s}, q_{s}^{-1}\right]$. Let ${ }_{\mathcal{A}} \mathbf{U}$ be the $\mathcal{A}$-subalgebra of $\mathbf{U}$ generated by elements $E_{i}^{(n)}, F_{i}^{(n)}, q^{h}$ for $i \in I, n \in \mathbf{Z}_{>0}, h \in d^{-1} P^{*}$.

Let us introduce a $\mathbf{Q}\left(q_{s}\right)$-algebra involutive automorphism $\vee$ and $\mathbf{Q}\left(q_{s}\right)$-algebra involutive anti-automorphisms $*$ and $\psi$ of $\mathbf{U}$ by

$$
\begin{gathered}
E_{i}^{\vee}=F_{i}, \quad F_{i}^{\vee}=E_{i}, \quad\left(q^{h}\right)^{\vee}=q^{-h}, \\
E_{i}^{*}=E_{i}, \quad F_{i}^{*}=F_{i}, \quad\left(q^{h}\right)^{*}=q^{-h}, \\
\psi\left(E_{i}\right)=q_{i}^{-1} t_{i}^{-1} F_{i}, \quad \psi\left(F_{i}\right)=q_{i}^{-1} t_{i} E_{i}, \quad \psi\left(q^{h}\right)=q^{h} .
\end{gathered}
$$

We define a $\mathbf{Q}$-algebra involutive automorphism ${ }^{-}$of $\mathbf{U}$ by

$$
\begin{array}{cl}
\overline{E_{i}}=E_{i}, & \overline{F_{i}}=F_{i}, \quad \overline{q^{h}}=q^{-h}, \\
\overline{a\left(q_{s}\right) u}=a\left(q_{s}^{-1}\right) \bar{u} & \text { for } a\left(q_{s}\right) \in \mathbf{Q}\left(q_{s}\right) \text { and } u \in \mathbf{U} .
\end{array}
$$

We define the coproduct $\Delta$ on $\mathbf{U}$ by

$$
\begin{gathered}
\Delta q^{h}=q^{h} \otimes q^{h}, \quad \Delta E_{i}=E_{i} \otimes t_{i}^{-1}+1 \otimes E_{i} \\
\Delta F_{i}=F_{i} \otimes 1+t_{i} \otimes F_{i} .
\end{gathered}
$$

Let us denote by $\Omega$ the $\mathbf{Q}$-algebra anti-automorphism *o- $\circ \vee$ of $\mathbf{U}$. We have

$$
\Omega\left(E_{i}\right)=F_{i}, \quad \Omega\left(F_{i}\right)=E_{i}, \quad \Omega\left(q^{h}\right)=q^{-h}, \quad \Omega\left(q_{s}\right)=q_{s}^{-1} .
$$

A U-module $M$ is called integrable if

(1) all $E_{i}, F_{i}(i \in I)$ are locally nilpotent, and

(2) it admits a weight space decomposition:

$$
M=\bigoplus_{\lambda \in P} M_{\lambda}, \quad \text { where } M_{\lambda}=\left\{u \in M \mid q^{h} u=q^{\langle h, \lambda\rangle} u \text { for all } h \in P^{*}\right\} .
$$

Let $\widetilde{\mathbf{U}}$ be the modified enveloping algebra [23, Part IV]. It is defined by

$$
\widetilde{\mathbf{U}}=\bigoplus_{\lambda \in P} \mathbf{U} a_{\lambda}, \quad \mathbf{U} a_{\lambda}=\mathbf{U} / \sum_{h \in P^{*}} \mathbf{U}\left(q^{h}-q^{\langle h, \lambda\rangle}\right) .
$$

Here the multiplication is given by

$$
a_{\lambda} x=x a_{\lambda-\xi} \quad \text { for } \xi \in \mathbf{U}_{\xi}, \quad a_{\lambda} a_{\mu}=\delta_{\lambda \mu} a_{\lambda},
$$

where $a_{\lambda}$ is considered as the image of 1 in the above definition of $\mathbf{U} a_{\lambda}$.

Let $\lambda, \mu \in P_{+}$. Let $V(\lambda)$ (resp. $V(-\mu)$ ) be the irreducible highest (resp. lowest) weight module of weight $\lambda$ (resp. $-\mu$ ) [23, §3.5]. Then there is a surjective homomorphism

$$
\mathbf{U} a_{\lambda-\mu} \ni u \longmapsto u\left(u_{\lambda} \otimes u_{-\mu}\right) \in V(\lambda) \otimes V(-\mu),
$$

where $u_{\lambda}$ (resp. $u_{-\mu}$ ) is a highest (resp. lowest) weight vector of $V(\lambda)$ (resp. $V(-\mu)$ ). 
2.3. Bilinear Form. In constructing our crystal base a key component is a variant of a bilinear form introduced by Drinfeld which characterizes the global crystal basis of ${ }_{\mathcal{A}} \mathbf{U}^{+}$. To introduce the form, first define an algebra structure on $\mathbf{U}^{+} \otimes \mathbf{U}^{+}$by

$$
\left(x_{1} \otimes x_{2}\right)\left(y_{1} \otimes y_{2}\right)=q_{s}^{\left(\text {wt } x_{2}, \text { wt } y_{1}\right)} x_{1} y_{1} \otimes x_{2} y_{2},
$$

where $x_{t}, y_{t}(t=1,2)$ are homogeneous. Let $r: \mathbf{U}^{+} \rightarrow \mathbf{U}^{+} \otimes \mathbf{U}^{+}$be the $\mathbf{Q}\left(q_{s}\right)$ algebra homomorphism defined by extending $r\left(E_{i}\right)=E_{i} \otimes 1+1 \otimes E_{i}(i \in I)$. By 23. 1.2.5], the algebra $\mathbf{U}^{+}$has a unique symmetric bilinear form $():, \mathbf{U}^{+} \times \mathbf{U}^{+} \rightarrow$ $\mathbf{Q}\left(q_{s}\right)$ satisfying $(1,1)=1$ and

$$
\begin{aligned}
& \left(E_{i}, E_{j}\right)=\delta_{i j} \frac{1}{\left(1-q_{i}^{-2}\right)}, \\
& \left(x, y y^{\prime}\right)=\left(r(x), y \otimes y^{\prime}\right), \quad\left(x x^{\prime}, y\right)=\left(x \otimes x^{\prime}, r(y)\right),
\end{aligned}
$$

where the form on $\mathbf{U}^{+} \otimes \mathbf{U}^{+}$is defined by $\left(x_{1} \otimes y_{1}, x_{2} \otimes y_{2}\right)=\left(x_{1}, x_{2}\right)\left(y_{1}, y_{2}\right)$.

For $i \in I$, introduce the unique $\mathbf{Q}\left(q_{s}\right)$-linear map $r_{i}: \mathbf{U}^{+} \rightarrow \mathbf{U}^{+}$given by $r_{i}(1)=$ $0, r_{i}\left(E_{j}\right)=\delta_{i j}$ for $j \in I$, and satisfying $r_{i}(x y)=q_{s}^{\left(\text {wt } y, \alpha_{i}\right)} r_{i}(x) y+x r_{i}(y)$ for all homogeneous $x, y \in \mathbf{U}^{+}([\mathrm{L} 3,1.2 .13])$. Similarly, introduce the unique $\mathbf{Q}\left(q_{s}\right)$-linear map ${ }_{i} r: \mathbf{U}^{+} \rightarrow \mathbf{U}^{+}$given by ${ }_{i} r(1)=0,{ }_{i} r\left(E_{j}\right)=\delta_{i j}$, and satisfying ${ }_{i} r(x y)=$ ${ }_{i} r(x) y+q_{s}^{\left(\text {wt } x, \alpha_{i}\right)} x_{i} r(y)$ for all homogeneous $x, y \in \mathbf{U}^{+}$.

From the definition the form satisfies

$$
\begin{aligned}
& \left(E_{i} y, x\right)=\left(1-q_{i}^{-2}\right)^{-1}\left(y,{ }_{i} r(x)\right), \\
& \left(y E_{i}, x\right)=\left(1-q_{i}^{-2}\right)^{-1}\left(y, r_{i}(x)\right) .
\end{aligned}
$$

2.4. Braid group action. For each $w \in \hat{W}$, there exists an $\mathbf{Q}\left(q_{s}\right)$-algebra automorphism $T_{w}$ [23, §39] (denoted there by $T_{w, 1}^{\prime \prime}$ ). Also, for any integrable $\mathbf{U}$-module $M$, there exists $\mathbf{Q}(q)$-linear map $T_{w}: M \rightarrow M$ satisfying $T_{w}(x u)=T_{w}(x) T_{w}(u)$ for $x \in \mathbf{U}, u \in M[23, \S 5]$. We denote $T_{s_{i}}$ by $T_{i}$ hereafter. By [23, 39.4.5] we have

$$
\Omega \circ T_{w} \circ \Omega=T_{w} .
$$

The definition of the automorphism $T_{w}$ of $\mathbf{U}$ can be extended to the case $w \in \widetilde{W}$ by setting

$$
\tau E_{i}=E_{\tau(i)}, \quad \tau F_{i}=F_{\tau(i)}, \quad \tau q^{h_{i}}=q^{h_{\tau(i)}}, \quad \tau q^{d}=q^{d} .
$$

2.5. Crystal bases. We briefly recall the notion of crystal bases. For the notion of (abstract) crystals and more details, we refer to [17, 2].

For $n \in \mathbf{Z}$ and $i \in I$, let us define an operator acting on any integrable $\mathbf{U}$-module $M$ by

$$
\begin{gathered}
\widetilde{F}_{i}^{(n)}=\sum_{k \geq \max (0,-n)} F_{i}^{(n+k)} E_{i}^{(k)} a_{k}^{n}\left(t_{i}\right), \\
\text { where } a_{k}^{n}\left(t_{i}\right)=(-1)^{k} q_{i}^{k(1-n)} t_{i}^{k} \prod_{\nu=1}^{k-1}\left(1-q_{i}^{n+2 \nu}\right) .
\end{gathered}
$$

And we set $\tilde{e}_{i}=F_{i}^{(-1)}, \tilde{f}_{i}=F_{i}^{(1)}$.

These operators are different from those used for the definition of crystal bases in [16], but give us the same crystal bases by [18, Proposition 6.1]. 
Let $\mathbf{A}_{0}=\left\{f\left(q_{s}\right) \in \mathbf{Q}\left(q_{s}\right) \mid f\right.$ is regular at $\left.q_{s}=0\right\}$. Let $\mathbf{A}_{\infty}=\overline{\mathbf{A}_{0}}$ be the image of $\mathbf{A}_{0}$ under - that is, the subring of $\mathbf{Q}\left(q_{s}\right)$ consisting of rational functions regular at $q_{s}=\infty$.

Definition 2.7. Let $M$ be an integrable $\mathbf{U}$-module. A pair $(\mathcal{L}, \mathcal{B})$ is called a crystal basis of $M$ if it satisfies

(1) $\mathcal{L}$ is a free $\mathbf{A}_{0}$-submodule of $M$ such that $M \cong \mathbf{Q}\left(q_{s}\right) \otimes_{\mathbf{A}_{0}} \mathcal{L}$,

(2) $\mathcal{L}=\bigoplus_{\lambda \in P} \mathcal{L}_{\lambda}$ where $\mathcal{L}_{\lambda}=\mathcal{L} \cap M_{\lambda}$ for $\lambda \in P$,

(3) $\mathcal{B}$ is a $\mathbf{Q}$-basis of $\mathcal{L} / q \mathcal{L} \cong \mathbf{Q} \otimes_{\mathbf{A}_{0}} \mathcal{L}$,

(4) $\tilde{e}_{i} \mathcal{L} \subset \mathcal{L}, \tilde{f}_{i} \mathcal{L} \subset \mathcal{L}$ for all $i \in I$,

(5) if we denote operators on $\mathcal{L} / q \mathcal{L}$ induced by $\tilde{e}_{i}, \tilde{f}_{i}$ by the same symbols, we have $\tilde{e}_{i} \mathcal{B} \subset \mathcal{B} \sqcup\{0\}, \tilde{f}_{i} \mathcal{B} \subset \mathcal{B} \sqcup\{0\}$,

(6) for any $b, b^{\prime} \in \mathcal{B}$ and $i \in I$, we have $b^{\prime}=\tilde{f}_{i} b$ if and only if $b=\tilde{e}_{i} b^{\prime}$.

We define functions $\varepsilon_{i}, \varphi_{i}: \mathcal{B} \rightarrow \mathbf{Z}_{\geq 0}$ by $\varepsilon_{i}(b)=\max \left\{n \geq 0 \mid \tilde{e}_{i}^{n} b \neq 0\right\}, \varphi_{i}(b)=$ $\max \left\{n \geq 0 \mid \tilde{f}_{i}^{n} b \neq 0\right\}$. We set $\tilde{e}_{i}^{\max } b=\tilde{e}_{i}^{\varepsilon_{i}(b)} b, \tilde{f}_{i}^{\max } b=\tilde{f}_{i}^{\varphi_{i}(b)} b$.

Let $M$ be an integrable $\mathbf{U}$-module with a crystal basis $(\mathcal{L}, \mathcal{B})$. Let ${ }^{-}$be an involution of an integrable $\mathbf{U}$-module $M$ satisfying $\overline{x u}=\bar{x} \bar{u}$ for any $x \in \mathbf{U}, u \in M$. Let ${ }_{\mathcal{A}} M$ be a ${ }_{\mathcal{A}} \mathbf{U}$-submodule of $M$ such that $\overline{\mathcal{A} M}={ }_{\mathcal{A}} M, u-\bar{u} \in\left(q_{s}-1\right)_{\mathcal{A}} M$ for $u \in{ }_{\mathcal{A}} M$. We say that $M$ has a global basis $\left(\mathcal{L}, \mathcal{B},{ }_{\mathcal{A}} M\right)$ if the following conditions are satisfied

(1) $M \cong \mathbf{Q}\left(q_{s}\right) \otimes_{\mathbf{Z}\left[q_{s}, q_{s}^{-1}\right]} \mathcal{A} \cong \mathbf{Q}\left(q_{s}\right) \otimes_{\mathbf{A}_{0}} \mathcal{L} \cong \mathbf{Q}\left(q_{s}\right) \otimes_{\mathbf{A}_{\infty}} \overline{\mathcal{L}}$,

(2) $\mathcal{L} \cap \overline{\mathcal{L}} \cap{ }_{\mathcal{A}} M \rightarrow \mathcal{L} \cap{ }_{\mathcal{A}} M / q_{s}\left(\mathcal{L} \cap{ }_{\mathcal{A}} M\right)$ is an isomorphism,

(3) $\mathcal{B} \subset \mathcal{L} \cap{ }_{\mathcal{A}} M / q_{s}\left(\mathcal{L} \cap{ }_{\mathcal{A}} M\right)$.

As a consequence of the definition, natural homomorphisms

$$
\begin{gathered}
\mathbf{A}_{0} \otimes_{\mathbf{z}}\left(\mathcal{L} \cap \overline{\mathcal{L}} \cap{ }_{\mathcal{A}} M\right) \rightarrow \mathcal{L}, \quad \mathbf{A}_{\infty} \otimes_{\mathbf{z}}\left(\mathcal{L} \cap \overline{\mathcal{L}} \cap{ }_{\mathcal{A}} M\right) \rightarrow \overline{\mathcal{L}}, \\
\mathbf{Z}\left[q_{s}, q_{s}^{-1}\right] \otimes \mathbf{z}\left(\mathcal{L} \cap \overline{\mathcal{L}} \cap{ }_{\mathcal{A}} M\right) \rightarrow{ }_{\mathcal{A}} M,
\end{gathered}
$$

are isomorphisms. We call the triple $\left(\mathcal{L}, \overline{\mathcal{L}},{ }_{\mathcal{A}} M\right)$ a balanced triple.

Let $G$ be the inverse isomorphism $\mathcal{L} \cap{ }_{\mathcal{A}} M / q_{s}\left(\mathcal{L} \cap{ }_{\mathcal{A}} M\right) \rightarrow \mathcal{L} \cap \overline{\mathcal{L}} \cap{ }_{\mathcal{A}} M$. Then $\{G(b) \mid b \in \mathcal{B}\}$ is a basis of $M$. It is called a global crystal basis of $M$. The above conditions imply $\overline{G(b)}=G(b)$.

$\mathbf{U}^{-}$(resp. $\left.\mathbf{U}^{+}\right)$has a global crystal basis $\left(\mathcal{L}(\infty), \mathcal{B}(\infty),{ }_{\mathcal{A}} \mathbf{U}^{-}\right)$(resp. $(\mathcal{L}(-\infty)$, $\left.\left.\mathcal{B}(-\infty),{ }_{\mathcal{A}} \mathbf{U}^{+}\right)\right)$. (It is not an integrable $\mathbf{U}$-module. But the above definitions has a modification.) For a dominant weight $\lambda \in P_{+}$, the irreducible highest weight module $V(\lambda)$ has a global crystal basis $\left(\mathcal{L}(\lambda), \mathcal{B}(\lambda),{ }_{\mathcal{A}} V(\lambda)\right)$ [16]. If $\lambda, \mu \in P_{+}$, then the tensor product $V(\lambda) \otimes V(-\mu)$ also has a global crystal basis $(\mathcal{L}(\lambda) \otimes$ $\left.\mathcal{L}(-\mu), \mathcal{B}(\lambda) \otimes \mathcal{B}(-\mu),{ }_{\mathcal{A}} V(\lambda) \otimes{ }_{\mathcal{A}} V(-\mu)\right)$, where the bar involution is defined by using the quasi $R$-matrix [23, Part IV]. Moreover, $\widetilde{\mathbf{U}}$ has a global crystal basis $\left(\mathcal{L}(\widetilde{\mathbf{U}}), \mathcal{B}(\widetilde{\mathbf{U}}),{ }_{\mathcal{A}} \widetilde{\mathbf{U}}\right)$ such that the homomorphism $(2.5)$ maps a global basis of $\widetilde{\mathbf{U}}$ to the union of that of $V(\lambda) \otimes V(-\mu)$ and 0 ([17, Theorem 2.1.2] and [23, Part IV]). Note that $\widetilde{\mathbf{U}}$ is not an integrable $\mathbf{U}$-module, and operators $\tilde{e}_{i}, \tilde{f}_{i}$ are defined only on $\mathcal{L}(\widetilde{\mathbf{U}}) / q_{s} \mathcal{L}(\widetilde{\mathbf{U}})$. In fact, they are defined so that $\mathcal{B}(\lambda) \otimes \mathcal{B}(-\mu) \rightarrow \mathcal{B}\left(\mathbf{U} a_{\lambda-\mu}\right) \subset \mathcal{B}(\widetilde{\mathbf{U}})$ is a strict embedding. Furthermore, the global basis is invariant under $*[17$, 4.3.2]. The proof given there also shows $\vee$ leaves the global basis invariant. We have $\mathcal{B}(\widetilde{\mathbf{U}})=\bigoplus_{\lambda \in P} \mathcal{B}\left(\mathbf{U} a_{\lambda}\right)$, the direct sum crystal bases of $\widetilde{\mathbf{U}} a_{\lambda}$. We define $\varepsilon^{*}, \varphi^{*}, \tilde{e}_{i}^{*}$, $\tilde{f}_{i}^{*}$ by $\varepsilon^{*}(b)=\varepsilon\left(b^{*}\right), \varphi^{*}(b)=\varphi\left(b^{*}\right), \tilde{e}_{i}^{*}(b)=\left(\tilde{e}_{i}\left(b^{*}\right)\right)^{*}$ and $\tilde{f}_{i}^{*}(b)=\left(\tilde{f}_{i}\left(b^{*}\right)\right)^{*}$. This 
another crystal structure on $\widetilde{\mathbf{U}}$ is called the star crystal structure. Occasionally we denote $\mathcal{B}(\widetilde{\mathbf{U}})$ simply by $\tilde{\mathcal{B}}$.

2.6. Braid group action and global crystal bases. We will recall results of 25. Let

$$
\mathbf{U}^{+}[i]=\left\{x \in \mathbf{U}^{+} \mid T_{i}(x) \in \mathbf{U}^{+}\right\}, \quad{ }^{*} \mathbf{U}^{+}[i]=\left\{x \in \mathbf{U}^{+} \mid T_{i}^{-1}(x) \in \mathbf{U}^{+}\right\} .
$$

By [23, 38.1] we have direct sum decompositions of vector spaces

$$
\mathbf{U}^{+}=\mathbf{U}^{+}[i] \oplus E_{i} \mathbf{U}^{+}, \quad \mathbf{U}^{+}={ }^{*} \mathbf{U}^{+}[i] \oplus \mathbf{U}^{+} E_{i} .
$$

Let ${ }^{i} \pi: \mathbf{U}^{+} \rightarrow \mathbf{U}^{+}[i], \pi^{i}: \mathbf{U}^{+} \rightarrow{ }^{*} \mathbf{U}^{+}[i]$ be the natural projections. We have the algebra isomorphism $T_{i}: \mathbf{U}^{+}[i] \rightarrow{ }^{*} \mathbf{U}^{+}[i]$. Then [25, Proposition 1.9] says

$$
T_{i}\left({ }^{i} \pi(\bar{x})\right)=\pi^{i}\left(\overline{T_{i}(x)}\right)
$$

for $x \in \mathbf{U}^{+}[i]$.

By [23, 14.3] ${ }^{i} \pi$ (resp. $\pi^{i}$ ) maps the global basis to the union of a basis of $\mathbf{U}^{+}[i]$ (resp. $\left.{ }^{*} \mathbf{U}^{+}[i]\right)$ and 0 . Then [25, Theorem 1.2] says that $T_{i}\left({ }^{i} \pi(G(b))\right)$, if it is nonzero, is equal to $\pi^{i}\left(G\left(b^{\prime}\right)\right)$ for some $b^{\prime}$, and the map $b \mapsto b^{\prime}$ gives a bijection between $\left\{b \in \mathcal{B}(\infty) \mid{ }^{i} \pi(G(b)) \neq 0\right\}$ and $\left\{b^{\prime} \in \mathcal{B}(\infty) \mid \pi^{i}\left(G\left(b^{\prime}\right)\right) \neq 0\right\}$. This result is based in part on an earlier result [32].

2.7. Affinization. Let $M$ be an integrable $\mathbf{U}^{\prime}$-module, and let $M=\bigoplus_{\lambda \in P_{\mathrm{cl}}} M_{\lambda}$ be its weight decomposition. We define a $\mathbf{U}$-module $M_{\text {aff }}$ by

$$
M_{\mathrm{aff}}=\bigoplus_{\lambda \in P} M_{\mathrm{cl}(\lambda)} .
$$

The action of $e_{i}$ and $f_{i}$ are defined by restricting to each summand, so that the canonical homomorphism cl: $M_{\mathrm{aff}} \rightarrow M$ is $\mathbf{U}^{\prime}$-linear. We define the $\mathbf{U}^{\prime}$-linear automorphism $z$ of $M_{\text {aff }}$ with weight $\delta$ by $\left(M_{\mathrm{aff}}\right)_{\lambda} \stackrel{\sim}{\rightarrow} M_{\mathrm{cl}(\lambda)}=M_{\mathrm{cl}(\lambda+\delta)} \stackrel{\sim}{\rightarrow}\left(M_{\mathrm{aff}}\right)_{\lambda+\delta}$.

Choose a section $s: P_{\mathrm{cl}} \rightarrow P$ of cl: $P \rightarrow P_{\mathrm{cl}}$ such that $s\left(\operatorname{cl}\left(\alpha_{i}\right)\right)=\alpha_{i}$ for any $i \in I_{0}=I \backslash\{0\}$. Then $M$ is embedded into $M_{\text {aff }}$ by $s$ as a vector space. We have an isomorphism of $\mathbf{U}^{\prime}$-modules

$$
M_{\mathrm{aff}} \simeq \mathbf{Q}\left(q_{s}\right)\left[z, z^{-1}\right] \otimes M .
$$

Here and $e_{i} \in \mathbf{U}^{\prime}$ and $f_{i} \in \mathbf{U}^{\prime}$ act on the right hand side by $z^{\delta_{i 0}} \otimes e_{i}$ and $z^{-\delta_{i 0}} \otimes f_{i}$. Similarly, for a crystal with weights in $P_{\mathrm{cl}}$, we can define its affinization $B_{\text {aff }}$ by

$$
B_{\mathrm{aff}}=\bigsqcup_{\lambda \in P} B_{\mathrm{cl}(\lambda)} .
$$

If an integrable $\mathbf{U}^{\prime}$-module $M$ has a crystal basis $(L, B)$, then its affinization $M_{\text {aff }}$ has a crystal basis $\left(L_{\text {aff }}, B_{\text {aff }}\right)$.

For $a \in \mathbf{Q}\left(q_{s}\right)$, we define the $\mathbf{U}^{\prime}$-module $M_{a}$ by

$$
M_{a}=M_{\mathrm{aff}} /(z-a) M_{\mathrm{aff}} .
$$


2.8. Extremal weight modules. A crystal $\mathcal{B}$ over $\mathbf{U}$ is called regular if, for any $J \varsubsetneqq I, \mathcal{B}$ is isomorphic (as a crystal over $\mathbf{U}\left(\mathfrak{g}_{J}\right)$ ) to the crystal associated with an integrable $\mathbf{U}\left(\mathfrak{g}_{J}\right)$-module. (It was called normal in [17].) Here $\mathbf{U}\left(\mathfrak{g}_{J}\right)$ is the subalgebra generated by $E_{j}, F_{j}(j \in J), q^{h}\left(h \in d^{-1} P^{*}\right)$. By [17], the affine Weyl group $\hat{W}$ acts on any regular crystal. The action $S$ is given by

$$
S_{s_{i}} b= \begin{cases}\tilde{f}_{i}^{\left\langle h_{i}, \mathrm{wt} b\right\rangle} b & \text { if }\left\langle h_{i}, \text { wt } b\right\rangle \geq 0, \\ \tilde{e}_{i}^{-\left\langle h_{i}, \mathrm{wt} b\right\rangle} b & \text { if }\left\langle h_{i}, \text { wt } b\right\rangle \leq 0\end{cases}
$$

for the simple reflection $s_{i}$. We denote $S_{s_{i}}$ by $S_{i}$ hereafter.

Definition 2.12. Let $M$ be an integrable $\mathbf{U}$-module. A vector $u \in M$ with weight $\lambda \in P$ is called extremal, if the following holds for all $w \in \hat{W}$ :

$$
\begin{cases}E_{i} T_{w} u=0 & \text { if }\left\langle h_{i}, w \lambda\right\rangle \geq 0 \\ F_{i} T_{w} u=0 & \text { if }\left\langle h_{i}, w \lambda\right\rangle \leq 0 .\end{cases}
$$

In this case, we define $S_{w} u$ so that

$$
S_{i} S_{w} u= \begin{cases}F_{i}^{\left(\left\langle h_{i}, w \lambda\right\rangle\right)} S_{w} u & \text { if }\left\langle h_{i}, w \lambda\right\rangle \geq 0, \\ E_{i}^{\left(-\left\langle h_{i}, w \lambda\right\rangle\right)} S_{w} u & \text { if }\left\langle h_{i}, w \lambda\right\rangle \leq 0 .\end{cases}
$$

This is well-defined, i.e., $S_{w} u$ depends only on $w$.

Similarly, for a vector $b$ of a regular crystal $B$ with weight $\lambda$, we say that $b$ is extremal if it satisfies

$$
\begin{cases}\tilde{e}_{i} S_{w} b=0 & \text { if }\left\langle h_{i}, w \lambda\right\rangle \geq 0 \\ \tilde{f}_{i} S_{w} b=0 & \text { if }\left\langle h_{i}, w \lambda\right\rangle \leq 0 .\end{cases}
$$

Lemma 2.14 ([30, Lemma 2.11]). Suppose that an integrable $\mathbf{U}$-module $M$ has a crystal basis $(\mathcal{L}, \mathcal{B})$. If $u \in \mathcal{L} \subset M$ is an extremal vector of weight $\lambda$ satisfying $b=u \bmod q \mathcal{L} \in \mathcal{B}$, then $b$ is an extremal vector, and we have

$$
S_{w} u=(-1)^{N_{+}^{\vee}} q^{-N_{+}} T_{w} u, \quad S_{w} b=S_{w} u \bmod q \mathcal{L} \quad \text { for all } w \in \hat{W},
$$

where $N_{+}=\sum_{\alpha \in \mathcal{R}_{+} \cap w^{-1}\left(\mathcal{R}_{-}\right)} \max ((\alpha, \lambda), 0)$, and $N_{+}^{\vee}$ is given by replacing $\alpha$ by $\alpha^{\vee}$.

For $\lambda \in P$, Kashiwara defined the $\mathbf{U}$-module $V(\lambda)$ generated by $u_{\lambda}$ with the defining relation that $u_{\lambda}$ is an extremal vector of weight $\lambda[17$ ]. It has a presentation

$$
V(\lambda)=\mathbf{U} a_{\lambda} / I_{\lambda}, \quad I_{\lambda}=\bigoplus_{b \in \mathcal{B}\left(\mathbf{U} a_{\lambda}\right) \backslash \mathcal{B}(\lambda)} \mathbf{Q}(q) G(b),
$$

where $\mathcal{B}(\lambda)=\left\{b \in \mathcal{B}\left(\mathbf{U} a_{\lambda}\right) \mid b^{*}\right.$ is extremal $\}$. (Recall that $\mathcal{B}(\widetilde{\mathbf{U}})$ is regular $\$ 2.5$, so extremal vectors make sense.) $I_{\lambda}$ is a left $\mathbf{U}$-module and $V(\lambda)$ has a crystal base $(\mathcal{L}(\lambda), \mathcal{B}(\lambda))$ together with a ${ }_{\mathcal{A}} \mathbf{U}$-submodule ${ }_{\mathcal{A}} V(\lambda)$ with a global crystal basis, naturally induced from those of $\mathbf{U} a_{\lambda}$. We have ${ }_{\mathcal{A}} V(\lambda)=\bigoplus_{b \in \mathcal{B}(\lambda)} \mathcal{A} G(b) \bmod I_{\lambda}$. By the construction of $V(\lambda)$,

$$
\left\{G(b) u_{\lambda} \mid b \in \mathcal{B}(\widetilde{\mathbf{U}})\right\} \backslash\{0\}=\left\{G(b) \bmod I_{\lambda} \mid b \in \mathcal{B}(\lambda)\right\} .
$$

By abuse of notation $\mathcal{B}(\lambda)$ is considered both as the crystal basis of $V(\lambda)$ and as the subset of the crystal basis of $\mathcal{B}\left(\mathbf{U} a_{\lambda}\right)$.

\footnotetext{
${ }^{1}$ He denoted it by $V^{\max }(\lambda)$.
} 
For any $w \in W, u_{\lambda} \mapsto S_{w^{-1}} u_{w \lambda}$ gives an isomorphism of $\mathbf{U}$-modules:

$$
V(\lambda) \stackrel{\sim}{\rightarrow} V(w \lambda) .
$$

This isomorphism sends the global basis to the global basis. Similarly, we have an isomorphism of crystals

$$
S_{w}^{*}: \mathcal{B}(\lambda) \stackrel{\sim}{\rightarrow} \mathcal{B}(w \lambda) .
$$

Here we regard $\mathcal{B}(\lambda)$ as a subcrystal of $\mathcal{B}(\widetilde{\mathbf{U}})$, and $S_{w}^{*}$ is the Weyl group action on $\mathcal{B}(\tilde{\mathbf{U}})$ with respect to the star crystal structure. Since $\mathcal{B}(\lambda) \subset \mathcal{B}\left(\mathbf{U} a_{\lambda}\right) \cong \mathcal{B}(\infty) \otimes$ $T_{\lambda} \otimes \mathcal{B}(-\infty)$ where $T_{\lambda}$ is the crystal with one element of weight $\lambda$, we can consider $\mathcal{B}(\lambda)$ as a subcrystal of $\mathcal{B}(\infty) \otimes T_{\lambda} \otimes \mathcal{B}(-\infty)$.

If $\lambda$ is dominant or anti-dominant, then $V(\lambda)$ is isomorphic to the highest weight module or the lowest weight module of weight $\lambda$, so in this case the notation is consistent. For $\lambda \notin P^{0}, \lambda$ is in the Tits cone $\bigcup_{w} w\left(P_{+}\right)$and so $V(\lambda)$ is isomorphic to a representation with a dominant or anti-dominant weight.

Theorem 2.15 ( $\left[18\right.$, Theorem 5.1, Corollary 5.2]). (i) For $\lambda \in P^{0}$, the weight of any extremal vector of $\mathcal{B}(\lambda)$ is contained in $\mathrm{cl}^{-1}(\operatorname{cl}(\hat{W} \lambda))$.

(ii) For any $\lambda \in P$, the weight of any vector of $\mathcal{B}(\lambda)$ is contained in the convex hull of $W \lambda$.

When $X_{N}^{(r)} \neq A_{2 n}^{(2)}$, we define the "fundamental weights of level zero" by setting

$$
\varpi_{i}=\Lambda_{i}-a_{i}^{\vee} \Lambda_{0} \in P^{0}, i \in I_{0} .
$$

When $X_{N}^{(r)}=A_{2 n}^{(2)}$ we set

$$
\begin{aligned}
& \varpi_{n}=2 \Lambda_{n}-\Lambda_{0} \in P^{0} \\
& \varpi_{i}=\Lambda_{i}-\Lambda_{0} \in P^{0}, i=1, \ldots, n-1 .
\end{aligned}
$$

We have $P_{\mathrm{cl}}^{0}=\operatorname{cl}\left(P^{0}\right)=\bigoplus_{i \in I_{0}} \mathbf{Z c l}\left(\varpi_{i}\right)$. We say that $\lambda$ is a "basic weight" if $\operatorname{cl}(\lambda)$ is $W_{\mathrm{cl}}$ conjugate to $\mathrm{cl}\left(\varpi_{i}\right)$ for some $i \in I_{0}$.

In the $A_{2 n}^{(2)}$ case, our choice of fundamental level zero weights is different than that of $[18]$. It is a simple check that both choices span the same $\mathbf{Z}$-lattice in $\mathfrak{h}$. It follows that the image of this lattice under $\mathrm{cl}$ is independent of the choice. Choosing a basis of $P_{\mathrm{cl}}^{0}$ amounts to fixing a Weyl chamber of $\left(\Delta_{\mathrm{cl}}, \mathfrak{h}_{\mathrm{cl}}^{* 0}\right)$. Since any two Weyl chambers are conjugate under the Weyl group action, it follows that our choice of $\varpi_{i}, i \in I_{0}$ give the same set of basic weights as that in [18.

In [18, §5], Kashiwara describes the structure of level zero fundamental representations corresponding the basic weights $\varpi_{i}\left(i \in I_{0}\right)$. Let us note

$$
\left(\varpi_{i}, \tilde{\alpha}_{j}\right)=\delta_{i j} d_{i}, \quad\left\{n \in \mathbf{Z} \mid \varpi_{i}+n \delta \in \hat{W} \varpi_{i}\right\}=\mathbf{Z} d_{i},
$$

where $d_{i}$ is as in (2.2). We obtain a $\mathbf{U}^{\prime}$-linear automorphism $z_{i}$ of $V\left(\varpi_{i}\right)$ of weight $d_{i} \delta$, which sends $u_{\varpi_{i}}$ to $u_{\varpi_{i}+d_{i} \delta}$. We define the "fundamental level zero representation" $\mathbf{U}^{\prime}-$ module $W\left(\varpi_{i}\right)$ by:

$$
W\left(\varpi_{i}\right)=V\left(\varpi_{i}\right) /\left(z_{i}-1\right) V\left(\varpi_{i}\right) .
$$

Theorem 2.16 ( 18, Theorem 5.15]). (i) $W\left(\varpi_{i}\right)$ is a finite-dimensional irreducible integrable $\mathbf{U}^{\prime}$ - module.

(ii) $W\left(\varpi_{i}\right)$ has a global crystal basis with a simple crystal, i.e., the weight of any extremal vector of $\mathcal{B}\left(W\left(\varpi_{i}\right)\right)$ is contained in $W_{\mathrm{cl}} \cdot \operatorname{cl}\left(\varpi_{i}\right)$ and $\# \mathcal{B}\left(W\left(\varpi_{i}\right)\right)_{\operatorname{cl}\left(\varpi_{i}\right)}=1$. 
(iii) For any $\mu \in \operatorname{wt}\left(V\left(\varpi_{i}\right)\right)$,

$$
W\left(\varpi_{i}\right)_{\mathrm{cl}(\mu)} \simeq V\left(\varpi_{i}\right)_{\mu} .
$$

(iv) $\operatorname{dim} W\left(\varpi_{i}\right)_{\mathrm{cl}\left(\varpi_{i}\right)}=1$.

(v) The weight of any extremal vector of $W\left(\varpi_{i}\right)$ belongs to $\hat{W} \cdot \operatorname{cl}\left(\varpi_{i}\right)$.

(vi) wt $\left(W\left(\varpi_{i}\right)\right)$ is the intersection of $\operatorname{cl}\left(\varpi_{i}\right)+Q_{\mathrm{cl}}$ and the convex hull of $\hat{W}$. $\operatorname{cl}\left(\varpi_{i}\right)$.

(vii) $\mathbf{Q}\left(q_{s}\right)\left[z_{i}^{1 / d_{i}}\right] \otimes_{\mathbf{Q}\left(q_{s}\right)\left[z_{i}\right]} V\left(\varpi_{i}\right) \simeq W\left(\varpi_{i}\right)_{\text {aff }}$, where the action of $z_{i}^{1 / d_{i}}$ on the left hand side corresponds to the action of $z$ on the right hand side as defined in s.7.

(viii) $V\left(\varpi_{i}\right)$ is isomorphic to the submodule $\mathbf{Q}\left(q_{s}\right)\left[z^{d_{i}}, z^{-d_{i}}\right] \otimes W\left(\varpi_{i}\right)$ of $W\left(\varpi_{i}\right)_{\mathrm{aff}}$ as a $\mathbf{U}$-module. Here we identify $W\left(\varpi_{i}\right)_{\text {aff }}$ with $\mathbf{Q}\left(q_{s}\right)\left[z, z^{-1}\right] \otimes W\left(\varpi_{i}\right)$ as in (2.9).

(ix) Any irreducible finite-dimensional integrable $\mathbf{U}^{\prime}$-module with $\operatorname{cl}\left(\varpi_{i}\right)$ as an extremal weight is isomorphic to $W\left(\varpi_{i}\right)_{a}$ for some $a \in \mathbf{Q}\left(q_{s}\right) \backslash\{0\}$.

2.9. Bilinear form characterizing $V(\lambda)$. We recall the following properties of $V(\lambda)$ with respect to a natural bilinear form.

Proposition 2.17 (]3, Proposition 4.1]). The extremal weight module $V(\lambda)$ has a unique bilinear form (, ) satisfying

$$
\begin{gathered}
\left(u_{\lambda}, G(b)\right)= \begin{cases}1 & \text { if } G(b)=u_{\lambda}, \\
0 & \text { otherwise }\end{cases} \\
(x u, v)=(u, \psi(x) v) \quad \text { for } x \in{ }_{\mathcal{A}} \mathbf{U}, u, v \in V(\lambda) .
\end{gathered}
$$

The following theorem gives a characterization of the global basis of $V(\lambda)$ with respect to the form:

Theorem 2.20 (30, Theorem 3]). (i) $\{G(b) \mid b \in \mathcal{B}(\lambda)\}$ is almost orthonormal for ( , ), that is, $\left(G(b), G\left(b^{\prime}\right)\right) \equiv \delta_{b b^{\prime}} \bmod q \mathbf{Z}[q]$.

(ii) $\{ \pm G(b) \mid b \in \mathcal{B}(\lambda)\}=\left\{u \in{ }_{\mathcal{A}} V(\lambda) \mid \bar{u}=u,(u, u) \equiv 1 \bmod q \mathbf{Z}[q]\right\}$.

For fundamental representations this result is due to [33].

Remark 2.21. By the ensuing discussion in $\S 3$, Theorem 2.20 holds in the nonsymmetric case also.

\section{An integral CRYstal base For ${ }_{\mathcal{A}} \mathbf{U}^{+}$}

In this section we construct an integral basis $B$ of ${ }_{\mathcal{A}} \mathbf{U}^{+}$such that $B \subset \overline{\mathcal{L}(-\infty)}$ and under the natural map $\pi: \overline{\mathcal{L}(-\infty)} \rightarrow \overline{\mathcal{L}(-\infty)} / q_{s}^{-1} \overline{\mathcal{L}(-\infty)}, \pi(B)=\mathcal{B}(-\infty)$. (In fact, we construct a series of bases parametrized by $p \in \mathbf{Z}$ with these properties.)

3.1. Root Vectors. We introduce root vectors in ${ }_{\mathcal{A}} \mathbf{U}^{+}$. Recall that we chose $\tilde{\omega}_{i} \in \widetilde{P} \triangleleft \widetilde{W}$ for each $i \in I_{0}$ (see $\S 2.1$ ). We choose $\tau_{i} \in \mathcal{T}$ so that $\tilde{\omega}_{i} \tau_{i}^{-1} \in \hat{W}$. Choosing a reduced expression for $\tilde{\omega}_{i} \tau_{i}^{-1}$ for each $i \in I_{0}$ we fix a reduced expression of $\tilde{\omega}_{n} \tilde{\omega}_{n-1} \ldots \tilde{\omega}_{1}$ :

$$
\tilde{\omega}_{n} \tilde{\omega}_{n-1} \ldots \tilde{\omega}_{1}=s_{i_{1}} s_{i_{2}} \ldots s_{i_{N}} \tau, \quad\left(\tau=\tau_{n} \ldots \tau_{1}\right) .
$$

We define a doubly infinite sequence

$$
\mathbf{h}=\left(\ldots, i_{-1}, i_{0}, i_{1}, \ldots\right)
$$


by setting $i_{k+N}=\tau\left(i_{k}\right)$ for $k \in \mathbf{Z}$. Note that for any integers $m<p$, the product $s_{i_{m}} s_{i_{m+1}} \ldots s_{i_{p}} \in \hat{W}$ is a reduced expression. We have

$$
\begin{array}{r}
\mathcal{R}_{>}=\left\{\alpha_{i_{0}}, s_{i_{0}}\left(\alpha_{i_{-1}}\right), s_{i_{0}} s_{i_{-1}}\left(\alpha_{i_{-2}}\right), \ldots\right\}, \\
\mathcal{R}_{<}=\left\{\alpha_{i_{1}}, s_{i_{1}}\left(\alpha_{i_{2}}\right), s_{i_{1}} s_{i_{2}}\left(\alpha_{i_{3}}\right), \ldots\right\} .
\end{array}
$$

Remark 3.2. Our definition of the PBW basis will depend on the sequence $\mathbf{h}$. In particular, it depends on the choice of the numbering $I_{0}=\{1,2, \ldots, n\}$. Almost all of the results in 112 which we will use are independent of the numbering. But when $X_{N}^{(r)}=A_{2 n}^{(2)}$, 12, Corollary 4.2.6] depends on our choice such that $\tilde{\omega}_{n}$ corresponding to the short root $\alpha_{n}$ appears first in (3.1). We choose our $\mathbf{h}$ to agree with that in 12. (Our vertex $n$ is labeled by 1 there.)

Set

$$
\beta_{k}= \begin{cases}s_{i_{0}} s_{i_{-1}} \ldots s_{i_{k+1}}\left(\alpha_{i_{k}}\right), & \text { if } k \leq 0 \\ s_{i_{1}} s_{i_{2}} \ldots s_{i_{k-1}}\left(\alpha_{i_{k}}\right), & \text { if } k>0 .\end{cases}
$$

Define a total order on $\mathcal{R}$ by setting

$$
\beta_{0}<\beta_{-1}<\beta_{-2} \cdots<\delta^{(1)}<\cdots<\delta^{(n)}<2 \delta^{(1)}<\cdots<\beta_{3}<\beta_{2}<\beta_{1},
$$

where $k \delta^{(i)}$ denotes $(k \delta, i) \in \mathcal{R}_{0}$.

We now define root vectors for each element of $\mathcal{R}_{>} \sqcup \mathcal{R}_{<}$.

$$
E_{\beta_{k}}= \begin{cases}T_{i_{0}}^{-1} T_{i_{-1}}^{-1} \ldots T_{i_{k+1}}^{-1}\left(E_{i_{k}}\right) & \text { if } k \leq 0, \\ T_{i_{1}} T_{i_{2}} \ldots T_{i_{k-1}}\left(E_{i_{k}}\right) & \text { if } k>0 .\end{cases}
$$

By [23, 40.1.3] these are in $\mathbf{U}^{+}$. As usual, set $F_{\beta}=\Omega\left(E_{\beta}\right)$ for all $\beta \in \mathcal{R}_{>} \sqcup \mathcal{R}_{<}$.

Remark 3.6. We note that the root vectors $E_{d_{i} k \delta \pm \alpha_{i}}$ are described explicitly by:

$$
E_{k d_{i} \delta+\alpha_{i}}=T_{\tilde{\omega}_{i}}^{-k} E_{i}(k \geq 0), E_{k d_{i} \delta-\alpha_{i}}=T_{\tilde{\omega}_{i}}^{k} T_{i}^{-1} E_{i}(k>0) .
$$

These are the Drinfeld generators for $\mathbf{U}$.

Having defined real root vectors, we define the imaginary root vectors. For $k>0$, $i \in I_{0}$, set

$$
\tilde{\psi}_{i, k d_{i}}=E_{k d_{i} \delta-\alpha_{i}} E_{\alpha_{i}}-q_{i}^{-2} E_{\alpha_{i}} E_{k d_{i} \delta-\alpha_{i}},
$$

and define elements $E_{i, k d_{i} \delta} \in \mathbf{U}^{+}$by the functional equation

$$
\left(q_{i}-q_{i}^{-1}\right) \sum_{k=1}^{\infty} E_{i, k d_{i} \delta} u^{k}=\log \left(1+\sum_{k=1}^{\infty}\left(q_{i}-q_{i}^{-1}\right) \tilde{\psi}_{i, k d_{i}} u^{k}\right) .
$$

We have

$$
\left[E_{i, k d_{i} \delta}, E_{j, l d_{j} \delta}\right]=0 \text {. }
$$

(For the untwisted case see [3]. For general case see [12, Theorem 5.3.2].)

For each $i \in I_{0}$ we introduce the "integral" imaginary root vectors ([9], [1]) by

$$
\sum_{k \geq 0} \tilde{P}_{i, k d_{i}} u^{k}= \begin{cases}\exp \left(\sum_{k \geq 1} \frac{E_{n, k \delta}}{[2 k]_{n}} u^{k}\right) & \text { if }\left(X_{N}^{(r)}, i\right)=\left(A_{2 n}^{(2)}, n\right), \\ \exp \left(\sum_{k \geq 1} \frac{E_{i, k d_{i} \delta}}{[k]_{i}} u^{k}\right) & \text { otherwise. }\end{cases}
$$


They satisfy the following recursive identity:

$$
\tilde{P}_{i, k d_{i}}= \begin{cases}\frac{1}{[2 k]_{n}} \sum_{s=1}^{k} q_{n}^{2(s-k)} \tilde{\psi}_{n, s} \tilde{P}_{n, k-s} & \text { if }\left(X_{N}^{(r)}, i\right)=\left(A_{2 n}^{(2)}, n\right), \\ \frac{1}{[k]_{i}} \sum_{s=1}^{k} q_{i}^{s-k} \tilde{\psi}_{i, s d_{i}} \tilde{P}_{i,(k-s) d_{i}} & \text { otherwise. }\end{cases}
$$

These definitions are based on the definition of imaginary root vectors which appeared for type $A_{1}^{(1)}$ in [9]. The $A_{2 n}^{(2)}$ case is generalized from [1] where it appears in the $A_{2}^{(2)}$ case.

Let $\mathbf{U}^{+}(>)$(resp. $\mathbf{U}^{+}(<), \mathbf{U}^{+}(0)$ ) be the $\mathbf{Q}\left(q_{s}\right)$-subalgebra of $\mathbf{U}^{+}$generated by the $E_{\beta_{k}}$ for $k \leq 0$ (resp. $E_{\beta_{k}}$ for $k>0, E_{i, k d_{i} \delta}$ for $k>0$ ).

The $\tilde{P}_{i, k d_{i}}\left(i \in I_{0}, k>0\right)$ are used to construct a basis of the imaginary part of $\mathbf{U}^{+}$as follows. Let $\mathbf{c}_{0}=\left(\rho^{(1)}, \rho^{(2)}, \ldots, \rho^{(n)}\right)$ be an $n$-tuple of partitions where each $\rho^{(i)}=\left(\rho_{1}^{(i)} \geq \rho_{2}^{(i)} \geq \ldots\right)$. For a partition $\rho$, we denote its transpose by $\rho^{t}$. For each $\rho^{(i)}$, define the Schur function in the $\tilde{P}_{i, k d_{i}}$ by

$$
S_{\rho^{(i)}}=\operatorname{det}\left(\tilde{P}_{i,\left(\rho^{(i)} \frac{t}{k}-k+m\right) d_{i}}\right)_{1 \leq k, m \leq t},
$$

where $t \geq l\left(\rho^{(i)^{t}}\right)$. This puts the $\tilde{P}_{i, k d_{i}}$ in the role of elementary symmetric functions. Note that in [6, [] the transpose of $S_{\rho^{(i)}}$ is considered, but this make no difference. Denote the product over $i \in I_{0}$ of $S_{\rho^{(i)}}$ by

$$
S_{\mathbf{c}_{0}}=\prod_{i=1}^{n} S_{\rho^{(i)}} .
$$

Definition 3.9. Let $\mathbf{c}_{+} \in \mathbf{N}^{\mathbf{Z}_{\leq 0}}$ and $\mathbf{c}_{-} \in \mathbf{N}^{\mathbf{Z}_{>0}}$ be functions which are almost everywhere 0 . Let $\mathbf{c}_{0}$ as above. Let $\mathcal{C}$ denote the set of all such triples $\mathbf{c}=$ $\left(\mathbf{c}_{+}, \mathbf{c}_{0}, \mathbf{c}_{-}\right)$.

For each $p$ and $\mathbf{c} \in \mathcal{C}$ we define:

$$
\begin{aligned}
& \mathbf{c}_{+_{p}}=(\mathbf{c}(p), \mathbf{c}(p-1), \mathbf{c}(p-2), \ldots) \\
& \text { and } \quad \mathbf{c}_{-p}=(\mathbf{c}(p+1), \mathbf{c}(p+2), \mathbf{c}(p+3), \ldots) .
\end{aligned}
$$

where the components $\mathbf{c}(j)$ are actually $\mathbf{c}_{+}(j)$ (resp. $\mathbf{c}_{-}(j)$ ) when $j \leq 0$ (resp. $j>0)$.

Define

$$
\begin{aligned}
& E_{\mathbf{c}_{+_{p}}}=E_{i_{p}}^{\mathbf{c}(p)} T_{i_{p}}^{-1}\left(E_{i_{p-1}}^{\mathbf{c}(p-1)}\right) T_{i_{p}}^{-1} T_{i_{p-1}}^{-1}\left(E_{i_{p-2}}^{\mathbf{c}(p-2)}\right) \cdots \\
& E_{\mathbf{c}_{-p}}=\cdots T_{i_{p+1}} T_{i_{p+2}}\left(E_{i_{p+3}}^{(\mathbf{c}(p+3))}\right) T_{i_{p+1}}\left(E_{i_{p+2}}^{(\mathbf{c}(p+2))}\right) E_{i_{p+1}}^{(\mathbf{c}(p+1))},
\end{aligned}
$$

where the exponents above are written $\mathbf{c}(j)$ when they should be $\mathbf{c}_{+}(j)$ or $\mathbf{c}_{-}(j)$ for $j \leq 0$ or $j>0$ respectively.

Note that when $p=0$, the monomials $E_{\mathbf{c}_{ \pm_{0}}}$ are formed by multiplying the $\left(E_{\beta_{k}}\right)^{\left(\mathbf{c}_{ \pm}(k)\right)}$ for real roots $\beta_{k} \in \mathcal{R}_{\gtrless}$ in the order (3.4). Also, in this case we will omit the subindex and write $\mathbf{c}_{ \pm}$instead of $\mathbf{c}_{ \pm_{0}}$ - there should be no confusion with the $\mathbf{c}_{ \pm}$above. 
For $\mathbf{c} \in \mathcal{C}, p \in \mathbf{Z}$ we define (cf. [23, 40.2.3]):

$$
\begin{aligned}
L(\mathbf{c}, p)= & \left(E_{i_{p}}^{(\mathbf{c}(p))} T_{i_{p}}^{-1}\left(E_{i_{p-1}}^{(\mathbf{c}(p-1))}\right) T_{i_{p}}^{-1} T_{i_{p-1}}^{-1}\left(E_{i_{p-2}}^{(\mathbf{c}(p-2))}\right) \cdots\right) \\
& \times T_{i_{p+1}} T_{i_{p+2}} \cdots T_{i_{0}}\left(S_{\mathbf{c}_{0}}\right) \\
& \times\left(\cdots T_{i_{p+1}} T_{i_{p+2}}\left(E_{i_{p+3}}^{(\mathbf{c}(p+3))}\right) T_{i_{p+1}}\left(E_{i_{p+2}}^{(\mathbf{c}(p+2))}\right) E_{i_{p+1}}^{(\mathbf{c}(p+1))}\right) \\
= & E_{\mathbf{c}_{+p}}\left(T_{i_{p+1}} T_{i_{p+2}} \cdots T_{i_{0}}\left(S_{\mathbf{c}_{0}}\right)\right) E_{\mathbf{c}_{-p}},
\end{aligned}
$$

for $p \leq 0$. For $p \geq 1$, we replace the middle part by $T_{i_{p}}^{-1} T_{i_{p-1}}^{-1} \ldots T_{i_{2}}^{-1} T_{i_{1}}^{-1}\left(S_{\mathbf{c}_{0}}\right)$.

Note that

$$
\begin{aligned}
& L(\mathbf{c}, p-1)=T_{i_{p}} L(\mathbf{c}, p) \text { if } \mathbf{c}(p)=0, \\
& L(\mathbf{c}, p+1)=T_{i_{p+1}}^{-1} L(\mathbf{c}, p) \text { if } \mathbf{c}(p+1)=0,
\end{aligned}
$$

When $p=0$ we will denote $L(\mathbf{c}, 0)$ by $B_{\mathbf{c}}$. In this case, each $\mathbf{c} \in \mathcal{C}$ indexes an element which we will call of "PBW-type" in $\mathbf{U}^{+}$:

$$
B_{\mathbf{c}}=E_{\mathbf{c}_{+}} \cdot S_{\mathbf{c}_{0}} \cdot E_{\mathbf{c}_{-}} .
$$

We will call $B_{\mathbf{c}}$ for $\mathbf{c} \in \mathcal{C}$ purely imaginary if $\mathbf{c}_{+}=\mathbf{c}_{-}=0$. In this case we may write $\mathbf{c}_{0}$ instead of $\mathbf{c}=\left(0, \mathbf{c}_{0}, 0\right)$.

For each $p \in \mathbf{Z}$, we define a partial ordering $\prec_{p}$ on $\{\mathbf{c} \mid \mathbf{c} \in \mathcal{C}\}$ by letting $\mathbf{c} \prec_{p} \mathbf{c}^{\prime}$ if and only if

$$
\mathbf{c}_{+_{p}} \leq \mathbf{c}_{+_{p}}^{\prime} \text { and } \quad \mathbf{c}_{-p} \leq \mathbf{c}_{-p}^{\prime} \text { and one of these is strict. }
$$

Here both $\leq$ are the lexicographic ordering from left to right. For example, the first inequality means either $\mathbf{c}=\mathbf{c}^{\prime}$ or $\mathbf{c}(p)=\mathbf{c}^{\prime}(p), \mathbf{c}(p-1)=\mathbf{c}^{\prime}(p-1), \ldots$, $\mathbf{c}(p-k+1)=\mathbf{c}^{\prime}(p-k+1)$ and $\mathbf{c}(p-k)<\mathbf{c}^{\prime}(p-k)$ for some $k \geq 0$. Note that

$$
\text { if } \mathbf{c}(p)=\mathbf{c}^{\prime}(p)=0 \text {, then } \mathbf{c} \prec_{p} \mathbf{c}^{\prime} \Leftrightarrow \mathbf{c} \prec_{p-1} \mathbf{c}^{\prime} \text {. }
$$

We now state the main the theorem of this section.

Theorem 3.13. (i) For each $p \in \mathbf{Z},\{L(\mathbf{c}, p) \mid \mathbf{c} \in \mathcal{C}\}$ is an almost orthonormal basis of the $\mathbf{Q}\left(q_{s}\right)$-vector space $\mathbf{U}^{+}$, i.e., $\left(L(\mathbf{c}, p), L\left(\mathbf{c}^{\prime}, p\right)\right) \in \delta_{\mathbf{c}, \mathbf{c}^{\prime}}+q_{s}^{-1} \mathbf{Z}\left[\left[q_{s}^{-1}\right]\right] \cap$ $\mathbf{Q}\left(q_{s}\right)$.

(ii) Let $p \in \mathbf{Z}$. The transition matrix between $\{L(\mathbf{c}, p) \mid \mathbf{c} \in \mathcal{C}\}$ and the global crystal basis of $\mathbf{U}^{+}$is upper-triangular with 1 's on the diagonal and with above diagonal entries in $q_{s}^{-1} \mathbf{Z}\left[q_{s}^{-1}\right]$.

The property (i) follows from (ii) and the almost orthonormality of the global crystal base [23, 14.2.3]. However, we will use (i) during the proof of (ii) and we prove it independently. Let us also remark that (ii) implies that $\{L(\mathbf{c}, p) \mid \mathbf{c} \in \mathcal{C}\}$ is a basis of the integral form ${ }_{\mathcal{A}} \mathbf{U}^{+}$. The proof of (i) will be given in $\$ 3.3$. We postpone that of (ii) until Sect. 5 .

3.2. Vertex subalgebras. A key part of describing the PBW basis is its reduction to "vertex subalgebras". The following proposition describes the $n$ "vertex" subalgebras of $\mathbf{U}=\mathbf{U}\left(X_{N}^{(k)}\right)$ :

Proposition 3.14. Let $i \in I_{0}$. Let $\mathbf{U}^{(i)}$ be the $\mathbf{Q}\left(q_{i}\right)$-subalgebra of $\mathbf{U}=\mathbf{U}\left(X_{N}^{(r)}\right)$ generated by

$$
\left\{E_{i}, E_{d_{i} \delta-\alpha_{i}}, F_{i}, F_{d_{i} \delta-\alpha_{i}}, K_{i}^{ \pm 1}, K_{d_{i} \delta}^{ \pm 1}\right\} .
$$


Then $\mathbf{U}^{(i)}$ is isomorphic as a $\mathbf{Q}(q)$-algebra to $\mathbf{U}\left(A_{1}^{(1)}\right)$ in all cases except when $X_{N}^{(r)}=A_{2 n}^{(2)}$ and $i=n$, i.e., $\left(\alpha_{i}, \alpha_{i}\right)=1$. The isomorphism $\mathbf{U}\left(A_{1}^{(1)}\right) \rightarrow \mathbf{U}^{(i)}$ is given by $E_{1} \mapsto E_{i}, E_{0} \mapsto E_{d_{i} \delta-\alpha_{i}}, F_{1} \mapsto F_{i}, F_{0} \mapsto F_{d_{i} \delta-\alpha_{i}}, q \mapsto q_{i}$. For $\left(X_{N}^{(r)}, i\right)=$ $\left(A_{2 n}^{(2)}, n\right), \mathbf{U}^{(i)}$ is isomorphic as a $\mathbf{Q}\left(q^{1 / 2}\right)$-algebra to $A_{2}^{(2)}$, and the isomorphism $A_{2}^{(2)} \rightarrow \mathbf{U}^{(i)}$ is given by $E_{0} \mapsto E_{\delta-2 \alpha_{n}}, F_{0} \mapsto F_{\delta-2 \alpha_{n}}, q^{1 / 2} \rightarrow q^{1 / 2}$. (In particular, $E_{\delta-2 \alpha_{n}}, F_{\delta-2 \alpha_{n}} \in \mathbf{U}^{(i)}$.)

Proof. For the untwisted case see [3]. In the twisted case, the result is due to [12]. Note that in 12] the quantum algebra of type $A_{2 n}^{(2)}$ is normalized differently (the invariant bilinear form on $\mathfrak{h}^{*}$ is 2 times the one here).

Next we have:

Proposition 3.15. For $i \in I_{0}, k>0$ we have $\tilde{P}_{i, k d_{i}} \in{ }_{\mathcal{A}} \mathbf{U}^{+}$.

Proof. This is proved as in [6, Corollary 2.2] for the symmetric case. In the $\left(X_{N}^{(r)}, i\right)=\left(A_{2 n}^{(2)}, n\right)$ case this follows from Proposition 3.14 and 11, Corollary 8.6].

Proposition 3.16. Let $\mathbf{c} \in \mathcal{C}$. We have $L(\mathbf{c}, p) \in{ }_{\mathcal{A}} \mathbf{U}^{+}$.

Proof. By [12, 6.3.2] (see 顿 for the untwisted case) we have $T_{i_{p+1}} T_{i_{p+2}} \cdots T_{i_{0}}\left(S_{\mathbf{c}_{0}}\right)$, $T_{i_{p}}^{-1} T_{i_{p-1}}^{-1} \ldots T_{i_{2}}^{-1} T_{i_{1}}^{-1}\left(S_{\mathbf{c}_{0}}\right) \in \mathbf{U}^{+}$. Since $T_{w}$ preserves ${ }_{\mathcal{A}} \mathbf{U}$, they are contained in ${ }_{\mathcal{A}} \mathbf{U} \cap \mathbf{U}^{+}={ }_{\mathcal{A}} \mathbf{U}^{+}$by Proposition 3.15. We also have $E_{\mathbf{c}_{ \pm p}} \in{ }_{\mathcal{A}} \mathbf{U}^{+}$by [23, 41.1.3].

Next we cite a key property of the $\tilde{P}_{i, k d_{i}}$ :

Proposition 3.17. Let $k>0$.

$$
\tilde{P}_{i, k d_{i}}= \begin{cases}E_{d_{i} \delta-\alpha_{i}}^{(k)} E_{i}^{(k)}+q_{s}^{-1} x & \text { if }\left(X_{N}^{(r)}, i\right) \neq\left(A_{2 n}^{(2)}, n\right), \\ E_{\delta-2 \alpha_{n}}^{(k)} E_{n}^{(2 k)}+q_{s}^{-1} x & \text { if }\left(X_{N}^{(r)}, i\right)=\left(A_{2 n}^{(2)}, n\right),\end{cases}
$$

where $x$ is a sum of terms $B_{\mathbf{c}}$ with coefficients in $\mathbf{Z}\left[q_{s}^{-1}\right]$ where for each term $\mathbf{c}_{+}, \mathbf{c}_{-} \neq 0$. Furthermore, for each such $\mathbf{c} \in \mathcal{C}$, only imaginary root vectors $P_{i, t d_{i}}$ with $0<t<k$ appear in $S_{\mathbf{c}_{0}}$.

Proof. This is derived as in [6, Proposition 2.2 and eq. (4.9)], where it appears for the untwisted affine case. For the $A_{2 n}^{(2)}$ this appears as a special case of [1, Theorem 8.5].

3.3. Almost orthonormality. The following proposition is central to our calculations.

Proposition 3.19. For $i, j \in I_{0}, k, k^{\prime}>0$ we have

$$
\left(\tilde{P}_{i, k d_{i}}, \tilde{P}_{j, k^{\prime} d_{j}}\right) \equiv \delta_{k, k^{\prime}} \delta_{i j} \quad\left(\bmod q_{s}^{-1} \mathbf{A}_{\infty}\right) .
$$

Proof. This result appears in the symmetric case in [6] and in the $A_{2}^{(2)}$ case it appears in [1]. In general, the proof is analogous to one of the previous cases. In the non-symmetric case where $i \neq j$, we may assume $a_{i j}=-1$ since the condition is symmetric in $i$ and $j$. The result then follows from the following identity:

$$
r_{j}\left(E_{i, k d_{i} \delta}\right)= \begin{cases}(-1)^{k+1} q_{j}^{-1}\left(1-q_{j}^{-2}\right) E_{k d_{i} \delta-\alpha_{j}} & \text { if } d_{j} \mid k d_{i} \\ 0 & \text { otherwise }\end{cases}
$$


which is derived from the following special case of 12, Theorem 5.3.2] (just as in the symmetric case the previous identity is derived in [6):

$$
\left[E_{i, k d_{i} \delta}, F_{j}\right]= \begin{cases}(-1)^{k+1} \frac{[r]_{q_{i}}}{r} K_{\alpha_{j}} E_{k d_{i} \delta-\alpha_{j}} & \text { if } d_{j} \mid k d_{i} \\ 0 & \text { otherwise. }\end{cases}
$$

Next we need the following result regarding the coproduct formula for the imaginary root vectors. For any algebra $A$, let $A_{+}$denote its augmentation ideal.

Proposition 3.22. Let $k>0, i \in I_{0}$. Then

$$
\begin{aligned}
r\left(\tilde{P}_{i, k d_{i}}\right)=\sum_{s=0}^{k} & \tilde{P}_{i, s d_{i}} \otimes \tilde{P}_{i,(k-s) d_{i}} \\
& + \text { terms in } \mathbf{U}^{+}(<)_{+} \mathbf{U}^{+}(0) \otimes \mathbf{U}^{+}(0) \mathbf{U}^{+}(>)_{+} .
\end{aligned}
$$

Proof. The proof of this follows from the relation between the braid group action and the coproduct (see [23, 3.1.5, 37.3.2]) by using Remark 3.6. In the untwisted case, the argument is given in [11. In general, the argument is identical.

We have the following result which is proved as in [6] (see [1] for the $A_{2}^{(2)}$ case):

Proposition 3.23. Let $\mathbf{c}_{0}, \mathbf{c}_{0}^{\prime}$ be two n-tuples of partitions as above. Then

$$
\left(S_{\mathbf{c}_{0}}, S_{\mathbf{c}_{0}^{\prime}}\right) \equiv \delta_{\mathbf{c}_{0}, \mathbf{c}_{\mathbf{o}}^{\prime}} \quad\left(\bmod q_{s}^{-1} \mathbf{A}_{\infty}\right) .
$$

Proof of Theorem $3.13(i)$. Let $\mathbf{c}, \mathbf{c}^{\prime} \in \mathcal{C}$. Suppose $p \geq 1$. We have

$$
T_{i_{p}}^{-1} T_{i_{p-1}}^{-1} \cdots T_{i_{2}}^{-1} T_{i_{1}}^{-1}\left(S_{\mathbf{c}_{0}}\right) \in \mathbf{U}^{+} \cap T_{i_{p}}^{-1}\left(\mathbf{U}^{+}\right) \text {. }
$$

as we already remarked in the proof of Proposition 3.16. By [23, 38.2.1] we have

$$
\begin{aligned}
& \left(T_{i_{p}}^{-1} T_{i_{p-1}}^{-1} \cdots T_{i_{2}}^{-1} T_{i_{1}}^{-1}\left(S_{\mathbf{c}_{0}}\right), T_{i_{p}}^{-1} T_{i_{p-1}}^{-1} \cdots T_{i_{2}}^{-1} T_{i_{1}}^{-1}\left(S_{\mathbf{c}_{0}^{\prime}}\right)\right) \\
& \quad=\left(T_{i_{p-1}}^{-1} \cdots T_{i_{2}}^{-1} T_{i_{1}}^{-1}\left(S_{\mathbf{c}_{0}}\right), T_{i_{p-1}}^{-1} \cdots T_{i_{2}}^{-1} T_{i_{1}}^{-1}\left(S_{\mathbf{c}_{0}^{\prime}}\right)\right) .
\end{aligned}
$$

By the induction and Proposition 3.23 these are equal to $\delta_{\mathbf{c}, \mathbf{c}^{\prime}}$ modulo $q_{s}^{-1} \mathbf{A}_{\infty}$.

We have by [23, 40.2.4]

$$
\left(L(\mathbf{c}, p), L\left(\mathbf{c}^{\prime}, p\right)\right)=\left(S_{\mathbf{c}_{0}}, S_{\mathbf{c}_{0}^{\prime}}\right) \prod_{s \in \mathbf{Z}}\left(E_{\alpha_{i_{s}}}^{(\mathbf{c}(s))}, E_{\alpha_{i_{s}}}^{\left(\mathbf{c}^{\prime}(s)\right)}\right) \equiv \delta_{\mathbf{c}, \mathbf{c}^{\prime}} \quad \bmod q_{s}^{-1} \mathbf{A}_{\infty}
$$

Moreover, we know $\left(L(\mathbf{c}, p), L\left(\mathbf{c}^{\prime}, p\right)\right) \in \mathbf{Z}\left[\left[q_{s}^{-1}\right]\right]$ since $L(\mathbf{c}, p), L\left(\mathbf{c}^{\prime}, p\right) \in{ }_{\mathcal{A}} \mathbf{U}^{+}$by 23. 14.2.6]. Hence we get the almost orthonormality.

For $p<0$, we have the same result thanks to

$$
T_{i_{p+1}} T_{i_{p+2}} \cdots T_{i_{0}}\left(S_{\mathbf{c}_{0}}\right) \in \mathbf{U}^{+} \cap T_{i_{p}}^{-1}\left(\mathbf{U}^{+}\right) .
$$

By (3.25) $\{L(\mathbf{c}, p)\}$ is linearly independent. The PBW theorem says that the dimension of a weight space of $\mathbf{U}^{+}$is equal to the number of $\mathbf{c}^{\prime}$ 's with the given weight. Thus it is a basis.

Corollary 3.26. Let $\mathcal{L}(-\infty)=\left\{x \in \mathbf{U}^{+} \mid(x, x) \in \mathbf{A}_{0}\right\}$. Then it is an $\mathbf{A}_{0^{-}}$ submodule of $\mathbf{U}^{+}$, and $\{\overline{L(\mathbf{c}, p)} \mid \mathbf{c} \in \mathcal{C}\}$ is its $\mathbf{A}_{0}$-basis for each $p \in \mathbf{Z}$.

Proof. The assertion follows from [23, 14.2.2]. 
Note that our proof of this statement, as well as that of Theorem 3.13(i), is independent of the existence of the global crystal basis. The above definition of $\mathcal{L}(-\infty)$ coincides with one in $[23,17.3 .3]$, while it was a characterizing property in [16, 5.1.4].

Proposition 3.27. For every $\mathbf{c} \in \mathcal{C}, p \in \mathbf{Z}$, there exist $b=b(\mathbf{c}, p) \in \mathcal{B}(-\infty)$ and $\operatorname{sgn}(\mathbf{c}, p)= \pm$ such that

$$
L(\mathbf{c}, p) \equiv \operatorname{sgn}(\mathbf{c}, p) G(b(\mathbf{c}, p)) \quad \bmod q_{s}^{-1} \overline{\mathcal{L}(-\infty)} .
$$

Proof. By [23, 14.2.2], we know that if $x \in{ }_{\mathcal{A}} \mathbf{U}^{+}$satisfies $(x, x) \in 1+q_{s}^{-1} \mathbf{A}_{\infty}$, then $x \in \overline{\mathcal{L}(-\infty)}$ and there exists $b \in \mathcal{B}(-\infty)$ such that $x \equiv \pm G(b) \bmod q_{s}^{-1} \overline{\mathcal{L}(-\infty)}$. The assertion follows from Theorem 3.13(i).

In Sect. 5 we will show $\operatorname{sgn}(\mathbf{c}, p)=1$.

Note that the map $\mathcal{C} \rightarrow \mathcal{B}(-\infty)$ given by $\mathbf{c} \mapsto b(\mathbf{c}, p)$ is bijective for any $p$, since both are bases.

Remark 3.29. For any $p, q \in \mathbf{Z}$ there exists a bijection $\mathbf{c} \in \mathcal{C} \leftrightarrow \mathbf{c}^{\prime} \in \mathcal{C}$ given by $b(\mathbf{c}, p)=b\left(\mathbf{c}^{\prime}, q\right)$ by Proposition 3.27. It is extremely interesting problem to give an explicit description of the bijection. For finite type $\mathfrak{g}$, the same construction gives the piecewise linear bijections 23, 42.1.3], which have been studied by various peoples. Let $b=b(\mathbf{c}, p-1)$ with $\mathbf{c}(p)=0$. By [32] we have $b(\mathbf{c}, p)=\tilde{e}_{i}^{* \varepsilon_{i}(b)} \tilde{f}_{i}^{\varphi_{i}(b)} b$, where $i=i_{p}$. This is a first step towards this problem.

3.4. Upper triangular property of the bar involution. This subsection is a small detour. We give a proof that the bar involution is upper triangular with respect to our basis $\{L(\mathbf{c}, p)\}$. For symmetric or type $A_{2}^{(2)}$, this together with [1, 6] gives us the elementary algebraic definition of the global crystal basis as explained in the Introduction. The reader in a hurry may skip the rest of this section.

We will need a "reordering lemma" to prove the upper-triangularity property of the bar action with respect to the ordering $\prec_{p}$.

Lemma 3.30. Let $p \in \mathbf{Z}$. Let $\mathbf{c}, \mathbf{c}^{\prime} \in \mathcal{C}$. Write

$$
L(\mathbf{c}, p) L\left(\mathbf{c}^{\prime}, p\right)=\sum_{\mathbf{c}^{\prime \prime}} a_{\mathbf{c}, \mathbf{c}^{\prime}}^{\mathbf{c}^{\prime \prime}} L\left(\mathbf{c}^{\prime \prime}, p\right),
$$

where $a_{\mathbf{c}, \mathbf{c}^{\prime}}^{\mathbf{c}^{\prime \prime}} \in \mathbf{Q}\left(q_{s}\right)$.

(i) Then for each $\mathbf{c}^{\prime \prime}$ in the above sum, $\mathbf{c}_{+_{p}}^{\prime \prime} \geq \mathbf{c}_{+_{p}}$ and $\mathbf{c}_{-{ }_{p}}^{\prime \prime} \geq \mathbf{c}_{-{ }_{p}}^{\prime}$ with respect to the lexicographical ordering.

(ii) Further, if $L(\mathbf{c}, p)=E_{\mathbf{c}_{+p}}$ (resp. $\left.E_{\mathbf{c}_{-p}}\right)$ and $L\left(\mathbf{c}^{\prime}, p\right)=E_{\mathbf{c}_{+_{p}}^{\prime}}$ (resp. $\left.E_{\mathbf{c}_{-p}^{\prime}}\right)$, then $\mathbf{c}_{+_{p}}^{\prime \prime}>\mathbf{c}_{+_{p}}$ (resp. $\mathbf{c}_{-{ }_{p}}^{\prime \prime}>\mathbf{c}_{-{ }_{p}}^{\prime}$ ) for each summand.

Proof. We prove this for $p=0$, noting that for $p \neq 0$ the proof is identical. Assume $L(\mathbf{c}, 0)=E_{\mathbf{c}_{+}} S_{\mathbf{c}_{0}} E_{\mathbf{c}_{-}}$and $L\left(\mathbf{c}^{\prime}, 0\right)=E_{\mathbf{c}_{+}^{\prime}} S_{\mathbf{c}_{0}^{\prime}} E_{\mathbf{c}_{-}^{\prime}}$. Write the expression

$$
S_{\mathbf{c}_{0}} E_{\mathbf{c}_{-}} E_{\mathbf{c}_{+}^{\prime}} S_{\mathbf{c}_{0}^{\prime}} E_{\mathbf{c}_{-}^{\prime}}=\sum_{\mathbf{b} \in \mathcal{C}} a_{\mathbf{c}, \mathbf{c}^{\prime}}^{\mathbf{b}} E_{\mathbf{b}_{+}} S_{\mathbf{b}_{0}} E_{\mathbf{b}_{-}} .
$$

Here and for the remainder of the section any structure constants (such as $a_{\mathbf{c}, \mathbf{c}^{\prime}}^{\mathbf{b}}$ ) are assumed to be in $\mathbf{Q}\left(q_{s}\right)$ unless otherwise stated. We have

$$
L(\mathbf{c}, 0) L\left(\mathbf{c}^{\prime}, 0\right)=\sum_{\mathbf{b}} a_{\mathbf{c}, \mathbf{c}^{\prime}}^{\mathbf{b}} E_{\mathbf{c}_{+}} E_{\mathbf{b}_{+}} S_{\mathbf{b}_{0}} E_{\mathbf{b}_{-}} .
$$


For a given summand, if $\mathbf{c}_{+}=0$ then clearly $\mathbf{c}_{+}^{\prime \prime}=\mathbf{b}_{+} \geq \mathbf{c}_{+}$in the Lemma. Assume $\mathbf{c}_{+}>0$. For any given $\mathbf{b}$ in the sum, we may assume $\mathbf{b}_{+}>0$ or else that summand also fulfills the requirement of the Lemma. Under the assumption that $\mathbf{b}_{+}>0$ and $\mathbf{c}_{+}>0$, the $\mathbf{c}_{+}^{\prime \prime} \geq \mathbf{c}_{+}$part in (i) and the $\mathbf{c}_{+}^{\prime \prime}>\mathbf{c}_{+}$part in (ii) follow if we check that for a fixed $\mathbf{b}_{+}$in $(3.33)$,

$$
E_{\mathbf{c}_{+}} E_{\mathbf{b}_{+}}=\sum_{\substack{\mathbf{d}_{+} \in \mathbf{N}^{\mathcal{R}}>\\ \mathbf{d}_{+}>\mathbf{c}_{+}}} a_{\mathbf{c}_{+}, \mathbf{b}_{+}}^{\mathbf{d}_{+}} E_{\mathbf{d}_{+}} .
$$

Let $k>k^{\prime}>0$. We have the following useful identity [19] (it was proved there in the finite type case, but the same proof works here):

$$
E_{\beta_{-k}} E_{\beta_{-k^{\prime}}}=q^{\left(\beta_{k}, \beta_{k^{\prime}}\right)} E_{\beta_{-k^{\prime}}} E_{\beta_{-k}}+\sum_{\mathbf{d}_{+}^{\prime}} a_{\mathbf{d}_{+}^{\prime}} E_{\mathbf{d}_{+}^{\prime}},
$$

where $\mathbf{d}_{+}^{\prime}(j)=0$ if $j \geq k$ or $j \leq k^{\prime}$. Note that this condition is equivalent to saying that $e_{-k^{\prime}}>\mathbf{d}_{+}^{\prime}>e_{-k}$, where $e_{k}$ is the tuple whose $j$-th position is $\delta_{j k}$.

Consider the set $S$ of all monomials of weight $\gamma=\operatorname{wt}\left(E_{\mathbf{c}_{+}} E_{\mathbf{b}_{+}}\right) \in Q_{+}$formed from the real root vectors $E_{\beta_{-k}}, k \geq 0$. $S$ is a finite set. We will order this set by a lexicographic order on the monomials, where $E_{\beta_{-k^{\prime}}}>E_{\beta_{-k}}$ if $k>k^{\prime} \geq 0$. In this ordering, a monomial $M$ is in the PBW order if and only if it is maximal among all monomials where $E_{\beta_{-k}}$ appears for all $k$ the same number of times as in $M$.

On the left hand side of (3.34), moving from left to right take the first root vector in $E_{\mathbf{c}_{+}} E_{\mathbf{b}_{+}}$which is out of $\mathrm{PBW}$ order, i.e., the first root vector which is larger than in it's immediate predecessor. Use (3.35) to reorder these two root vectors. By (3.35), we obtain a linear combination of monomials from $S$, each greater in lexicographic order than $E_{\mathbf{c}_{+}} E_{\mathbf{b}_{+}}$. Repeating this for each summand, and taking into account that $S$ is a finite set, we ultimately obtain a linear combination of elements of $S$, each of which is maximal among monomials formed from the same root vectors. Thus, each is in PBW form and also larger than $E_{\mathbf{c}_{+}}$in lexicographic order, so that we obtain $E_{\mathbf{c}_{+}} E_{\mathbf{b}_{+}}$in the form of (3.34) as required.

Proposition 3.36. Let $\mathbf{c} \in \mathcal{C}, p \in \mathbf{Z}$. Then

$$
\overline{L(\mathbf{c}, p)}=L(\mathbf{c}, p)+\sum_{\mathbf{c} \prec p \mathbf{c}^{\prime}} a_{\mathbf{c}, \mathbf{c}^{\prime}} L\left(\mathbf{c}^{\prime}, p\right),
$$

where $a_{\mathbf{c}, \mathbf{c}^{\prime}} \in \mathbf{Q}\left(q_{s}\right)$.

Proof. We first prove

(a): Fix $\mathbf{c}_{0}$ and $q$. Suppose that 3.37$)$ is true for $T_{i_{q+1}} T_{i_{q+2}} \cdots T_{i_{0}}\left(S_{\mathbf{c}_{0}}\right)(q \leq$ $0)$, or $T_{i_{q}}^{-1} T_{i_{q-1}}^{-1} \cdots T_{i_{1}}^{-1}\left(S_{\mathbf{c}_{0}}\right)(q \geq 1)$. Let $p \geq q$. If $\mathbf{c}$ satisfies $\mathbf{c}_{-p}=0$, $\mathbf{c}_{+_{q}}=0$ and $\mathbf{c}_{0}$ is the given one, 3.37 ) is also true for $L(\mathbf{c}, p)$. Furthermore, the condition $\mathbf{c} \prec_{p} \mathbf{c}^{\prime}$ can be replaced by the stronger condition $\mathbf{c}_{+_{p}}<\mathbf{c}_{+_{p}}^{\prime}$. (The other condition $(0=) \mathbf{c}_{-{ }_{p}} \leq \mathbf{c}_{-{ }_{p}}^{\prime}$ is trivially satisfied.)

We are considering

$$
\begin{gathered}
L(\mathbf{c}, p)=E_{i_{p}}^{(\mathbf{c}(p))} \times T_{i_{p}}^{-1}\left(E_{i_{p-1}}^{(\mathbf{c}(p-1))}\right) \times T_{i_{p}}^{-1} T_{i_{p-1}}^{-1}\left(E_{i_{p-2}}^{(\mathbf{c}(p-2))}\right) \times \cdots \\
\cdots \times T_{i_{p}}^{-1} \cdots T_{i_{q+2}}^{-1}\left(E_{i_{q+1}}^{(\mathbf{c}(q+1))}\right) \times T_{i_{p+1}} T_{i_{p+2}} \cdots T_{i_{0}}\left(S_{\mathbf{c}_{0}}\right) .
\end{gathered}
$$


(When $p \geq 1$, the last part is $T_{i_{p}}^{-1} T_{i_{p-1}}^{-1} \ldots T_{i_{2}}^{-1} T_{i_{1}}^{-1}\left(S_{\mathbf{c}_{0}}\right)$.) We prove the assertion by the induction on $p$. When $p=q$, (3.37) is true by the assumption.

First assume $\mathbf{c}(p)=0$. We consider $L(\mathbf{c}, p-1)=T_{i_{p}} L(\mathbf{c}, p)$ (see (3.10)). By the induction hypothesis, we have

$$
\overline{T_{i_{p}} L(\mathbf{c}, p)}=\overline{L(\mathbf{c}, p-1)}=L(\mathbf{c}, p-1)+\sum_{\mathbf{c}_{+_{p-1}}<\mathbf{c}_{+_{p-1}}^{\prime}} a_{\mathbf{c}, \mathbf{c}^{\prime}}^{p-1} L\left(\mathbf{c}^{\prime}, p-1\right) .
$$

(We put the superscript $p-1$ in order to clarify its dependence on $p-1$ in this part. For the other part, $p$ will be fixed, and there will be no confusion.) We apply the composition $T_{i_{p}}^{-1} \circ \pi^{i_{p}}$ to both sides. By (2.8), the left hand side becomes ${ }^{i_{p}} \pi(\overline{L(\mathbf{c}, p)})$, which is equal to $\overline{L(\mathbf{c}, p)}$ modulo $E_{i_{p}} \mathbf{U}^{+}$. For the right hand side, we use (3.10). We get

$$
\overline{L(\mathbf{c}, p)} \in L(\mathbf{c}, p)+\sum_{\substack{\mathbf{c}_{+_{p-1}<\mathbf{c}_{+p-1}^{\prime}} \\ \mathbf{c}^{\prime}(p)=0}} a_{\mathbf{c}, \mathbf{c}^{\prime}}^{p-1} L\left(\mathbf{c}^{\prime}, p-1\right)+E_{i_{p}} \mathbf{U}^{+} .
$$

The condition $\mathbf{c}^{\prime}(p)=0$ comes from $\pi^{i_{p}}\left(L\left(\mathbf{c}^{\prime}, p-1\right)\right) \neq 0$. (Otherwise, $L\left(\mathbf{c}^{\prime}, p-1\right) \in$ $\mathbf{U}^{+} E_{i_{p}}$ and $\pi^{i_{p}}\left(L\left(\mathbf{c}^{\prime}, p-1\right)\right)=0$. $)$ The part in $E_{i_{p}} \mathbf{U}^{+}$is a linear combination of $L\left(\mathbf{c}^{\prime \prime}, p\right)$ 's with $\mathbf{c}^{\prime \prime}(p)>0$. They satisfy $\mathbf{c}_{+, p}<\mathbf{c}_{+, p}^{\prime \prime}$ since $\mathbf{c}(p)=0$. The summation in the second term can be replaced as $\sum_{\mathbf{c}_{+_{p}}<\mathbf{c}_{+p}^{\prime}, \mathbf{c}^{\prime}(p)=0}$ by (3.12). Thus we have the assertion under the assumption $\mathbf{c}(p)=0$.

Next we assume $\mathbf{c}(p)>0$. Let us define $\tilde{\mathbf{c}}$ by setting $\tilde{\mathbf{c}}(p)=0$ and all other entries are the same as $\mathbf{c}$. We have

$$
L(\mathbf{c}, p)=E_{i_{p}}^{(\mathbf{c}(p))} L(\tilde{\mathbf{c}}, p) .
$$

Since $\tilde{\mathbf{c}}(p)=0$, we have just proved

$$
\overline{L(\tilde{\mathbf{c}}, p)}=L(\tilde{\mathbf{c}}, p)+\sum_{\tilde{\mathbf{c}}_{+_{p}}<\tilde{\mathbf{c}}_{+p}^{\prime}} a_{\tilde{\mathbf{c}}, \tilde{\mathbf{c}}^{\prime}}^{p} L\left(\tilde{\mathbf{c}}^{\prime}, p\right) .
$$

Therefore

$$
\overline{L(\mathbf{c}, p)}=E_{i_{p}}^{(\mathbf{c}(p))} \overline{L(\tilde{\mathbf{c}}, p)}=L(\mathbf{c}, p)+\sum_{\tilde{\mathbf{c}}_{+p}<\tilde{\mathbf{c}}_{+p}^{\prime}} a_{\tilde{\mathbf{c}}, \tilde{\mathbf{c}}^{\prime}}^{p}\left[\begin{array}{c}
\mathbf{c}(p)+\tilde{\mathbf{c}}^{\prime}(p) \\
\mathbf{c}(p)
\end{array}\right]_{q_{i}} L\left(\mathbf{c}^{\prime}, p\right),
$$

where $\mathbf{c}^{\prime}$ is defined by setting $\mathbf{c}^{\prime}(p)=\tilde{\mathbf{c}}^{\prime}(p)+\mathbf{c}(p)$ and other entries are the same as $\tilde{\mathbf{c}}^{\prime}$. We have $\tilde{\mathbf{c}}_{+_{p}}<\tilde{\mathbf{c}}_{+_{p}}^{\prime} \Longleftrightarrow \mathbf{c}_{+_{p}}^{\prime}<\mathbf{c}_{+_{p}}$, and therefore the assertion.

Similarly we have

(a'): Fix $\mathbf{c}_{0}$ and $q$. Suppose that (3.37) is true for $T_{i_{q+1}} T_{i_{q+2}} \cdots T_{i_{0}}\left(S_{\mathbf{c}_{0}}\right)$ $(q \leq 0)$, or $T_{i_{q}}^{-1} T_{i_{q-1}}^{-1} \cdots T_{i_{1}}^{-1}\left(S_{\mathbf{c}_{0}}\right)(q \geq 1)$. Let $p \leq q$. If $\mathbf{c}$ satisfies $\mathbf{c}_{+_{p}}=0$, $\mathbf{c}_{-q}=0$ and $\mathbf{c}_{0}$ is the given one, (3.37) is also true for $L(\mathbf{c}, p)$. Furthermore, the condition $\mathbf{c} \prec_{p} \mathbf{c}^{\prime}$ can be replaced by the stronger condition $\mathbf{c}_{-p}<\mathbf{c}_{-p}^{\prime}$. (The other condition $(0=) \mathbf{c}_{+_{p}} \leq \mathbf{c}_{+_{p}}^{\prime}$ is trivially satisfied.)

Our next task is to show

(b): Fix $\mathbf{c}_{0}$. Suppose that (3.37) is true for $S_{\mathbf{c}_{0}}$. Then it is also true for $L(\mathbf{c}, p)$ with $\mathbf{c}_{0}$ is the given one.

By (a), (a'), if (3.37) is true for $S_{\mathbf{c}_{0}}$, then it is also true for $T_{i_{p+1}} T_{i_{p+2}} \cdots T_{i_{0}}\left(S_{\mathbf{c}_{0}}\right)$ $(p \leq 0)$, or $T_{i_{p}}^{-1} T_{i_{p-1}}^{-1} \cdots T_{i_{1}}^{-1}\left(S_{\mathbf{c}_{0}}\right)(p \geq 1)$. (The conditions on $\mathbf{c}_{ \pm, p}, \mathbf{c}_{ \pm, q}$ are vacuous 
since $\mathbf{c}_{ \pm}=0$.) This is a special case of (b). We return back to general $L(\mathbf{c}, p)$ as in (b). We decompose it as $L(\mathbf{c}, p)=L\left(\mathbf{c}_{+_{p}}, p\right) L\left(\mathbf{c}_{0}, p\right) L\left(\mathbf{c}_{-p}, p\right)$ where $\mathbf{c}_{ \pm p}, \mathbf{c}_{0}$ are understood as elements of $\mathcal{C}$ by setting other entries as 0 . By (a) and the above special case of (b), we have

$$
\overline{L\left(\mathbf{c}_{+_{p}}, p\right) L\left(\mathbf{c}_{0}, p\right)}=L\left(\mathbf{c}_{+_{p}}, p\right) L\left(\mathbf{c}_{0}, p\right)+\sum_{\mathbf{c}_{+p}<\mathbf{c}_{+, p}^{\prime}} a_{\mathbf{c}_{+p}, \mathbf{c}^{\prime}} L\left(\mathbf{c}^{\prime}, p\right) .
$$

By (a') we have

$$
\overline{L\left(\mathbf{c}_{-p}, p\right)}=L\left(\mathbf{c}_{-p}, p\right)+\sum_{\mathbf{c}_{-p}<\mathbf{c}_{-p}^{\prime \prime}} a_{\mathbf{c}_{-p}, \mathbf{c}^{\prime \prime}} L\left(\mathbf{c}^{\prime \prime}, p\right) .
$$

Note that the assumption of $\left(\mathbf{a}^{\prime}\right)$ is trivially satisfied since $\mathbf{c}_{0}=0$ in this case. Therefore

$$
\begin{aligned}
\overline{L(\mathbf{c}, p)}=L(\mathbf{c}, p)+ & \sum_{\mathbf{c}_{+p}<\mathbf{c}_{+, p}^{\prime}} a_{\mathbf{c}_{+p}, \mathbf{c}^{\prime}} L\left(\mathbf{c}^{\prime}, p\right) L\left(\mathbf{c}_{-p}, p\right) \\
& +\sum_{\mathbf{c}_{-p}<\mathbf{c}_{-p}^{\prime \prime}} a_{\mathbf{c}_{-p}, \mathbf{c}^{\prime \prime}} L\left(\mathbf{c}_{+_{p}}, p\right) L\left(\mathbf{c}_{0}, p\right) L\left(\mathbf{c}^{\prime \prime}, p\right) \\
& \quad+\sum_{\mathbf{c}_{+_{p}}<\mathbf{c}_{+, p}^{\prime}, \mathbf{c}_{-p}<\mathbf{c}_{-p}^{\prime \prime}} a_{\mathbf{c}_{+p}, \mathbf{c}^{\prime}} a_{\mathbf{c}_{-p}, \mathbf{c}^{\prime \prime}} L\left(\mathbf{c}^{\prime}, p\right) L\left(\mathbf{c}^{\prime \prime}, p\right) .
\end{aligned}
$$

By Lemma 3.30, $L\left(\mathbf{c}^{\prime}, p\right) L\left(\mathbf{c}_{-p}, p\right)=\sum_{\mathbf{d}_{\mathbf{d}}} a_{\mathbf{c}^{\prime}, \mathbf{c}_{-p}}^{\mathbf{d}} L(\mathbf{d}, p)$ where the summation is over $\mathbf{d}$ satisfying $\mathbf{c}_{+_{p}}^{\prime} \leq \mathbf{d}_{+_{p}}, \mathbf{c}_{-_{p}} \leq \mathbf{d}_{-_{p}}$. Since $\mathbf{c}_{+_{p}}<\mathbf{c}_{+, p}^{\prime}$, we have $\mathbf{c}_{+_{p}}<\mathbf{d}_{+_{p}}$, and hence $\mathbf{c} \prec p$ d. (Recall that one of the inequalities must be strict in the definition of $\prec_{p}$.) The other two summations can be handled in the same way, and we have the assertion (b).

Next we replace the inequality $\mathbf{c} \prec_{p} \mathbf{c}^{\prime}$ by a further stronger inequality in a special case.

(c): Consider the case $p=0$. Let $\mathbf{c}_{k} \in \mathcal{C}$ be such that $L\left(\mathbf{c}_{k}, 0\right)$ is equal to $E_{d i \delta-\alpha_{i}}^{(k)}$ if $\left(X_{N}^{(r)}, i\right) \neq\left(A_{2 n}^{(2)}, n\right)$, and $E_{\delta-2 \alpha_{n}}^{(k)}$ if $\left(X_{N}^{(r)}, i\right)=\left(A_{2 n}^{(2)}, n\right)$. Then (3.37) is true for $L\left(\mathbf{c}_{k}, 0\right)$ with $\mathbf{c}_{k} \prec p \mathbf{c}^{\prime}$ replaced by $(0=) \mathbf{c}_{k,+}<\mathbf{c}_{+}^{\prime}$ and $\mathbf{c}_{k,-}<\mathbf{c}_{-}^{\prime}$.

Here we have written $\mathbf{c}_{k,-}$ instead of $\left(\mathbf{c}_{k}\right)_{-}$, etc.

We check

$$
\overline{L\left(\mathbf{c}_{k}, 0\right)}=L\left(\mathbf{c}_{k}, 0\right)+\sum_{\substack{\mathbf{c}_{k,+}<\mathbf{c}_{+}^{\prime} \\ \mathbf{c}_{k,-}<\mathbf{c}_{-}^{\prime}}} a_{\mathbf{c}_{k}, \mathbf{c}^{\prime}} L\left(\mathbf{c}^{\prime}, 0\right) .
$$

By (a'), we already know $\mathbf{c}_{k,-}<\mathbf{c}_{-}^{\prime}$. Thus we only need to show $\mathbf{c}_{+}^{\prime} \neq 0$. Suppose that $\mathbf{c}_{+}^{\prime}=0$, and hence $L\left(\mathbf{c}^{\prime}, 0\right)=S_{\mathbf{c}_{0}^{\prime}} E_{\mathbf{c}_{-}^{\prime}}$. Then wt $\left(L\left(\mathbf{c}^{\prime}, 0\right)\right)=\operatorname{wt}\left(S_{\mathbf{c}_{0}^{\prime}}\right)+$ $\operatorname{wt}\left(E_{\mathbf{c}_{-}^{\prime}}\right)$, which is equal to $k\left(d_{i} \delta-\alpha_{i}\right)$ if $\left(X_{N}^{(r)}, i\right) \neq\left(A_{2 n}^{(2)}, n\right)$, and $k\left(\delta-2 \alpha_{n}\right)$ if $\left(X_{N}^{(r)}, i\right)=\left(A_{2 n}^{(2)}, n\right)$. Since wt $\left(S_{\mathbf{c}_{0}^{\prime}}\right) \in \mathbf{N} \delta, E_{\mathbf{c}_{-}^{\prime}}$ must be a product of $E_{l d_{i} \delta-\alpha_{i}}^{\left(c_{l}\right)}$ when $\left(X_{N}^{(r)}, i\right) \neq\left(A_{2 n}^{(2)}, n\right)$, and $E_{(2 l-1) \delta-2 \alpha_{n}}^{\left(c_{l}\right)}, E_{m \delta-\alpha_{n}}^{\left(d_{m}\right)}$ when $\left(X_{N}^{(r)}, i\right)=\left(A_{2 n}^{(2)}, n\right)$ (in an appropriate order). When $\left(X_{N}^{(r)}, i\right) \neq\left(A_{2 n}^{(2)}, n\right)$, we have $\sum c_{l}=k$ and $\operatorname{wt}\left(S_{\mathbf{c}_{0}^{\prime}}\right)+$ $\sum l c_{l}=k$. These equations simultaneously hold only if $c_{1}=k, c_{l}=0(l \neq 1)$, and wt $\left(S_{\mathbf{c}_{0}^{\prime}}\right)=0$ (i.e., $\mathbf{c}_{0}^{\prime}=0$ ). When $\left(X_{N}^{(r)}, i\right)=\left(A_{2 n}^{(2)}, n\right)$, we have $\sum 2 c_{l}+\sum d_{m}=2 k$, 
$\sum(2 l-1) c_{l}+\sum m d_{m}+\operatorname{wt}\left(S_{\mathbf{c}_{0}^{\prime}}\right)=k$. Thus $\sum(2 l-2) c_{l}+\sum\left(m-\frac{1}{2}\right) d_{m}+\operatorname{wt}\left(S_{\mathbf{c}_{0}^{\prime}}\right)=0$, and hence $c_{l}=0(l \geq 2), d_{m}=0$, wt $\left(S_{\mathbf{c}_{0}^{\prime}}\right)=0$. We get $c_{1}=k$. In both cases, we have $L\left(\mathbf{c}^{\prime}, 0\right)=E_{\mathbf{c}_{k,-}}$, which is impossible.

The following together with (b) completes the proof.

(d): (3.37) is true for $S_{\mathbf{c}_{0}}$ for any $\mathbf{c}_{0}$. Namely $\overline{S_{\mathbf{c}_{0}}}=S_{\mathbf{c}_{0}}+\sum_{0<\mathbf{c}_{ \pm}^{\prime}} a_{\mathbf{c}_{0}, \mathbf{c}^{\prime}} L\left(\mathbf{c}^{\prime}, 0\right)$ (The inequality $\mathbf{c}_{0} \prec_{p} \mathbf{c}^{\prime}$ is equivalent to the above inequalities since $\mathrm{wt}\left(L\left(\mathbf{c}^{\prime}, 0\right)\right) \in$ $\mathbf{Z} \delta$.)

We prove (d) by the induction on the length of $\mathbf{c}_{0}$. When $\ell\left(\mathbf{c}_{0}\right)=0$, we understand $S_{\mathbf{c}_{0}}=1$, and the assertion is trivial. We assume $\left(X_{N}^{(r)}, i\right) \neq\left(A_{2 n}^{(2)}, n\right)$ now, but the argument works even when $\left(X_{N}^{(r)}, i\right)=\left(A_{2 n}^{(2)}, n\right)$ with obvious modifications. By Proposition 3.17 we have

$$
\tilde{P}_{i, k d_{i}}=E_{d_{i} \delta-\alpha_{i}}^{(k)} E_{\alpha_{i}}^{(k)}+\sum_{0<\mathbf{c}_{ \pm}^{\prime}} a_{\mathbf{c}^{\prime}} L\left(\mathbf{c}^{\prime}, 0\right) .
$$

Taking - of both sides of this equality we have

$$
\begin{aligned}
\overline{\tilde{P}_{i, k d_{i}}} & =\overline{E_{d_{i} \delta-\alpha_{i}}^{(k)}} E_{\alpha_{i}}^{(k)}+\sum_{0<\mathbf{c}_{ \pm}^{\prime}} \overline{a_{\mathbf{c}^{\prime}} L\left(\mathbf{c}^{\prime}, 0\right)} \\
& =E_{d_{i} \delta-\alpha_{i}}^{(k)} E_{\alpha_{i}}^{(k)}+\sum_{\mathbf{c}_{k, \pm}<\mathbf{c}_{ \pm}^{\prime \prime}} a_{\mathbf{c}, \mathbf{c}^{\prime \prime}} L\left(\mathbf{c}^{\prime \prime}, 0\right) E_{\alpha_{i}}^{(k)}+\sum_{0<\mathbf{c}_{ \pm}^{\prime}} \overline{a_{\mathbf{c}^{\prime}} L\left(\mathbf{c}^{\prime}, 0\right)},
\end{aligned}
$$

where we have used (c) in the second equality. By Lemma 3.30, $L\left(\mathbf{c}^{\prime \prime}, 0\right) E_{\alpha_{i}}^{(k)}$ is a sum of $L(\mathbf{d}, 0)$ with $\mathbf{c}_{+}^{\prime \prime} \leq \mathbf{d}_{+}$. (The other inequality $0 \leq \mathbf{d}_{-}$is trivially satisfied.) Since $(0=) \mathbf{c}_{k,+}<\mathbf{c}_{+}^{\prime \prime}$, we have $0<\mathbf{d}_{+}$.

Recall that by Proposition 3.17 the purely imaginary part of $L\left(\mathbf{c}^{\prime}, 0\right)$ is a polynomial in $\tilde{P}_{i, t d_{i}}$ with $0<t<k$. By the induction hypothesis, (3.37) is true for this polynomial. Then it is also true for $L\left(\mathbf{c}^{\prime}, 0\right)$ by (b). Thus (3.37) is true for $\tilde{P}_{i, k d_{i}}$. By Lemma 3.30, the assertion is also true for $S_{\mathbf{c}_{0}}$ if it is a polynomial in $\tilde{P}_{i, t d_{i}}$ with $t \leq k$.

When $\mathfrak{g}$ is symmetric or type $A_{2}^{(2)}$ we know that the $\operatorname{set}\{L(\mathbf{c}, 0) \mid \mathbf{c} \in \mathcal{C}\}$ is an $\mathcal{A}$-basis of ${ }_{\mathcal{A}} \mathbf{U}^{+}$from $[6]$ and [1]. Using this result, we can obtain the more general

Lemma 3.39. For each $p \in \mathbf{Z}$, the set $\{L(\mathbf{c}, p) \mid \mathbf{c} \in \mathcal{C}\}$ is an $\mathcal{A}$-basis of ${ }_{\mathcal{A}} \mathbf{U}^{+}$.

Proof. First note that

$$
\{L(\mathbf{c}, 0) \mid \mathbf{c} \in \mathcal{C}, \mathbf{c}(0)=0\}
$$

is an $\mathcal{A}$-basis of ${ }_{\mathcal{A}} \mathbf{U}^{+} \cap \mathbf{U}^{+}\left[i_{0}\right]$. To see this take any $x \in{ }_{\mathcal{A}} \mathbf{U}^{+} \cap \mathbf{U}^{+}\left[i_{0}\right]$. Since the lemma holds for $p=0$, we have $x=\sum_{\mathbf{c}^{\prime} \in \mathcal{C}} a_{\mathbf{c}^{\prime}} L\left(\mathbf{c}^{\prime}, 0\right)$ with $a_{\mathbf{c}^{\prime}} \in \mathcal{A}$. Now $x \in \mathbf{U}^{+}\left[i_{0}\right] \Leftrightarrow$ each $L\left(\mathbf{c}^{\prime}, 0\right) \in \mathbf{U}^{+}\left[i_{0}\right]$ since $\mathbf{U}^{+}=\mathbf{U}^{+}\left[i_{0}\right] \oplus E_{i_{0}} \mathbf{U}^{+}$and clearly each $L\left(\mathbf{c}^{\prime}, 0\right)$ is in one of these direct summands. Consider the image of (3.40) under the two maps $T_{i_{0}}$ and $*$ respectively. We have

$$
T_{i_{0}}:\{L(\mathbf{c}, 0) \mid \mathbf{c} \in \mathcal{C}, \mathbf{c}(0)=0\} \stackrel{1-1}{\longrightarrow}\{L(\mathbf{c},-1) \mid \mathbf{c} \in \mathcal{C}, \mathbf{c}(0)=0\},
$$

as well as

$$
*:\{L(\mathbf{c}, 0) \mid \mathbf{c} \in \mathcal{C}, \mathbf{c}(0)=0\} \stackrel{1-1}{\longrightarrow}\left\{L(\mathbf{c}, 0)^{*} \mid \mathbf{c} \in \mathcal{C}, \mathbf{c}(0)=0\right\} .
$$


Since both $*$ and $T_{i_{0}}$ leave ${ }_{\mathcal{A}} \mathbf{U}^{+}$invariant, and both take $\mathbf{U}^{+}\left[i_{0}\right]$ isomorphically to ${ }^{*} \mathbf{U}^{+}\left[i_{0}\right]$ we obtain that the two sets

$$
\{L(\mathbf{c},-1) \mid \mathbf{c} \in \mathcal{C}, \mathbf{c}(0)=0\}, \quad\left\{L(\mathbf{c}, 0)^{*} \mid \mathbf{c} \in \mathcal{C}, \mathbf{c}(0)=0\right\},
$$

are both $\mathcal{A}$-bases of ${ }^{*} \mathbf{U}^{+}\left[i_{0}\right] \cap{ }_{\mathcal{A}} \mathbf{U}^{+}$. This implies that the two sets

$$
\left\{L(\mathbf{c},-1) E_{i_{0}}^{(k)} \mid \mathbf{c} \in \mathcal{C}, \mathbf{c}(0)=0, k \in \mathbf{N}\right\}, \quad\left\{L(\mathbf{c}, 0)^{*} E_{i_{0}}^{(k)} \mid \mathbf{c} \in \mathcal{C}, \mathbf{c}(0)=0, k \in \mathbf{N}\right\},
$$

span the same $\mathcal{A}$-submodules of ${ }_{\mathcal{A}} \mathbf{U}^{+}$. The right hand set is a $\mathcal{A}$-basis of ${ }_{\mathcal{A}} \mathbf{U}^{+}$ since it's the image under $*$ of a $\mathcal{A}$-basis of ${ }_{\mathcal{A}} \mathbf{U}^{+}$. Therefore the left hand set is a $\mathcal{A}$-basis of ${ }_{\mathcal{A}} \mathbf{U}^{+}$, but this set is exactly $\{L(\mathbf{c},-1) \mid \mathbf{c} \in \mathcal{C}\}$. With the same reasoning, an induction gives the lemma for all $p<0$. A similar argument works for $p>0$.

From Proposition 3.36 and Lemma 3.39 we deduce using [23, 24.2.1]:

Theorem 3.45. Let $\mathfrak{g}$ be affine of symmetric or of type $A_{2}^{(2)}$.

(i) For any $p \in \mathbf{Z}, \mathbf{c} \in \mathcal{C}$ there is a unique $b(\mathbf{c}, p) \in{ }_{\mathcal{A}} \mathbf{U}^{+}$such that

(a) $\overline{b(\mathbf{c}, p)}=b(\mathbf{c}, p)$

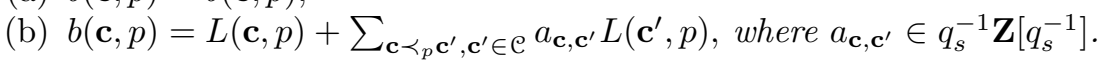

(ii) The $\mathbf{Z}$-homomorphism

$$
\mathcal{L}(-\infty) \cap \overline{\mathcal{L}(-\infty)} \cap{ }_{\mathcal{A}} \mathbf{U}^{+} \rightarrow \mathcal{L}(-\infty) \cap{ }_{\mathcal{A}} \mathbf{U}^{+} / q_{s}^{-1}\left(\mathcal{L}(-\infty) \cap{ }_{\mathcal{A}} \mathbf{U}^{+}\right)
$$

is an isomorphism.

For $p, q \in \mathbf{Z}$, there exists a bijection $\mathbf{c} \in \mathcal{C} \leftrightarrow \mathbf{c}^{\prime} \in \mathcal{C}$ such that $b(\mathbf{c}, p)= \pm b\left(\mathbf{c}^{\prime}, q\right)$ by the proof of [23, 14.2.3]. We will show $b(\mathbf{c}, p)=G(b)$ for some $b \in \mathcal{B}(-\infty)$ in Sect. 5, but it is desirable to have a proof of + -sign in the above equality, independent of the existence of the global crystal basis.

\section{Crystal structure of $\widetilde{\mathbf{U}}$}

Let $P_{+}^{0}=\sum_{i \in I_{0}} \mathbf{N} \varpi_{i}$. Let $\lambda=\sum_{i} \lambda_{i} \varpi_{i} \in P_{+}^{0}$. Let $G_{\lambda}=\prod_{i} G L_{\lambda_{i}}(\mathbf{C})$ and $\operatorname{Irr} G_{\lambda}$ the set of irreducible representations of $G_{\lambda}$. Let $\breve{B}(\lambda)$ be the crystal of $\bigotimes_{i} V\left(\varpi_{i}\right)^{\otimes \lambda_{i}}$. In [18, §13] Kashiwara conjectures a description of the crystal structure of $V(\lambda)$ in terms of $\operatorname{Irr} G_{\lambda}$ and $\breve{B}(\lambda)$. This conjecture was proved in affine case. It can now be checked for arbitrary type modulo sign using the results of the previous section. The modification is straightforward, but we recall the proof for the sake of reader. We also give a Peter-Weyl type description of $\mathcal{B}(\widetilde{\mathbf{U}})$ which is conjectured in [18, §13] (see also [31]), but not proved in [5, 30].

The sign ambiguity will be removed in Sect. 5 based on results in this section. Thus returning back to this section again, we get Kashiwara's conjecture.

Remark 4.1. In the previous section we constructed an integral basis of ${ }_{\mathcal{A}} \mathbf{U}^{+}$. In this section, for the purposes of calculation we replace the basis elements $B_{\mathbf{c}}, \mathbf{c} \in \mathcal{C}$ with $B_{\mathbf{c}}^{+}=\overline{B_{\mathbf{c}}^{*}}, \mathbf{c} \in \mathcal{C}$. We also replace the $F_{\alpha}=\Omega\left(E_{\alpha}\right), \alpha \in \mathcal{R}^{+}$which appeared in the previous section, with $F_{\alpha}^{-}=\overline{E_{\alpha}^{\vee}}, \alpha \in \mathcal{R}^{+}$. Additionally, we define the integral basis of ${ }_{\mathcal{A}} \mathbf{U}^{-}$given by $B_{\mathbf{c}}^{-}=\overline{B_{\mathbf{c}}^{\vee}}$. Let $\tilde{P}_{i,-k d_{i}}=\overline{\tilde{P}_{i, k d_{i}}^{\vee}}$. There are two reasons to do this. First, applying the operator allows us to work in $\mathcal{L}( \pm \infty)$ rather than $\overline{\mathcal{L}( \pm \infty)}$. Second, the operators $*$ and $\vee$ reverse the root orderings in $B_{\text {c }}^{ \pm}$ 
and so we are able to work with highest weight (relative to the map cl) level zero representations instead of lowest weight level zero representations.

Definition 4.2. For $\lambda=\sum_{i} \lambda_{i} \varpi_{i} \in P_{+}^{0}$ let

$$
\mathbf{N}^{\mathcal{R}_{0}}(\lambda)=\left\{\mathbf{c}_{0}=\left(\rho^{(1)}, \ldots, \rho^{(n)}\right) \mid \rho^{(i)} \text { a partition, } \ell\left(\rho^{(i)}\right) \leq \lambda_{i}, i=1, \ldots, n\right\} .
$$

This is identified with the set of irreducible polynomial representations of $G_{\lambda}$, and the set of $n$-tuples $\left(s_{\rho^{(1)}}, \ldots, s_{\rho^{(n)}}\right)$, where $s_{\rho^{(i)}}$ is a Schur function in variables $z_{i, 1}$, $\ldots, z_{i, \lambda_{i}}$.

\subsection{Preparatory results.}

Proposition 4.3. (i) For any $\lambda \in P_{+}^{0}$, any vector in $\mathcal{B}(\lambda)$ is connected to an extremal weight vector of the form $b_{1} \otimes t_{\lambda} \otimes u_{-\infty}$, where $b_{1}$ is purely imaginary with respect to the crystal base.

(ii) Furthermore, all such possible $b_{1} \in \mathcal{B}(\infty)$ are given by $\operatorname{sgn}\left(\mathbf{c}_{0}, 0\right) S_{\mathbf{c}_{0}}^{-} u_{\infty} \bmod$ $q_{s} \mathcal{L}(\infty)$ where $\mathbf{c}_{0} \in \mathbf{N}^{\mathcal{R}_{0}}(\lambda)$.

Proof. (i) By [18, Proof of Theorem 5.1] any vector is connected to an extremal weight vector of the form $b_{1} \otimes t_{\lambda} \otimes u_{-\infty}$, where wt $\left(b_{1}\right)=-k \delta$. Using the basis of $\S 3$ to express $b_{1}$, we take $\operatorname{sgn}(\mathbf{c}, 0) B_{\mathbf{c}}^{-}=b_{1} \bmod q_{s} \mathcal{L}(\infty)$, for some c. Assume that $B_{\mathbf{c}}^{-}$isn't purely imaginary. Since wt $\left(B_{\mathbf{c}}^{-}\right)=-k \delta$, and $B_{\mathbf{c}_{+}}^{-}$(resp. $\left.B_{\mathbf{c}_{-}}^{-}\right)$consists only of terms in root vectors with positive real part (resp. negative real part), it follows $\mathbf{c}_{-} \neq 0$. By Theorem 2.15 we have $B_{\mathbf{c}}^{-} u_{\lambda}=0$. However, by assumption $B_{\mathbf{c}}^{-} u_{\lambda} \in \mathcal{L}(\lambda)$ such that $B_{\mathbf{c}}^{-} u_{\lambda} \neq 0 \bmod q_{s} \mathcal{L}(\lambda)$. This is a contradiction.

(ii) From Proposition 3.17 we have

$$
\tilde{P}_{i,-k d_{i}} u_{\lambda}= \begin{cases}F_{d_{i} \delta-\alpha_{i}}^{-(k)} F_{i}^{(k)} u_{\lambda} & \text { if }\left(X_{N}^{(r)}, i\right) \neq\left(A_{2 n}^{(2)}, n\right), \\ F_{\delta-2 \alpha_{n}}^{-(k)} F_{n}^{(2 k)} u_{\lambda} & \text { if }\left(X_{N}^{(r)}, i\right)=\left(A_{2 n}^{(2)}, n\right) .\end{cases}
$$

Here $u_{\lambda}$ generates $V(\lambda)$. Since the weights of $V(\lambda)$ are in the convex hull of $W \lambda$ (see Theorem 2.15), this implies that $\tilde{P}_{i,-k d_{i}} u_{\lambda}=0$ for $k>\lambda_{i}$. Note that for any $i, \ell\left(\rho^{(i)}\right) \leq \lambda_{i} \Longleftrightarrow \rho^{(i)^{t}} \leq \lambda_{i}$. Since the $\tilde{P}_{i,-k d_{i}}$ all commute, considering the top row of the determinant $S_{\mathbf{c}_{0}}^{-}$, we have $S_{\mathbf{c}_{0}}^{-} u_{\lambda}=0$ for $\mathbf{c}_{0} \notin \mathbf{N}^{\mathcal{R}_{0}}(\lambda)$.

Let $\tilde{\alpha}_{i} \in \widetilde{Q}$ as in $\$ 2.1$ and let $S_{\tilde{\alpha}_{i}}$ be the corresponding operator in Definition 2.12 .

Lemma 4.5. Let $\lambda=\sum_{i} \lambda_{i} \varpi_{i} \in P_{+}^{0}$. Let $V(\lambda)$ be the extremal weight module generated by $u_{\lambda}$.

$$
\begin{aligned}
S_{\tilde{\alpha}_{i}} u_{\lambda} & = \begin{cases}F_{d_{i} \delta-\alpha_{i}}^{-\left(\lambda_{i}\right)} F_{\alpha_{i}}^{\left(\lambda_{i}\right)} u_{\lambda} & \text { if }\left(X_{N}^{(r)}, i\right) \neq\left(A_{2 n}^{(2)}, n\right) \\
F_{\left.\delta-2 \alpha_{n}\right)}^{-\left(\lambda_{n}\right)} F_{\alpha_{n}}^{\left(2 \lambda_{n}\right)} u_{\lambda} & \text { if }\left(X_{N}^{(r)}, i\right)=\left(A_{2 n}^{(2)}, n\right)\end{cases} \\
& =\tilde{P}_{i,-\lambda_{i} d_{i}} u_{\lambda}
\end{aligned}
$$

Proof. The second equality follows from the first equality and (4.4). We prove the first equality after applying $\circ \vee$. (See the above remark.)

First consider the case $\left(X_{N}^{(r)}, i\right) \neq\left(A_{2 n}^{(2)}, n\right)$. We have an identity $\tilde{\alpha}_{i}=s_{d_{i} \delta-\alpha_{i}} s_{i}=$ $\tilde{\omega}_{i} s_{i} \tilde{\omega}_{i}^{-1} s_{i}$ in the affine Weyl group (see $(2.3)$ ), where $s_{d_{i} \delta-\alpha_{i}}$ is the reflection with 
respect to $d_{i} \delta-\alpha_{i}$. Then

$$
\begin{aligned}
S_{\tilde{\alpha}_{i}} u_{-\lambda} & =S_{\tilde{\omega}_{i}} S_{i} S_{\tilde{\omega}_{i}^{-1}} S_{i} u_{-\lambda}=S_{\tilde{\omega}_{i}^{\prime}} S_{i} S_{\tilde{\omega}_{i}^{\prime-1}} E_{\alpha_{i}}^{\left(\lambda_{i}\right)} u_{-\lambda} \text { where } \tilde{\omega}_{i}^{\prime}=\tilde{\omega}_{i} s_{i} \\
& =S_{\tilde{\omega}_{i}^{\prime}} E_{\alpha_{i}}^{\left(\lambda_{i}\right)} S_{\tilde{\omega}_{i}^{\prime-1}} E_{\alpha_{i}}^{\left(\lambda_{i}\right)} u_{-\lambda}=q^{-N_{+}}(-1)^{N_{+}^{\vee}} T_{\tilde{\omega}_{i}^{\prime}}\left(E_{\alpha_{i}}^{\left(\lambda_{i}\right)}\right) T_{\tilde{\omega}_{i}^{\prime}}\left(S_{\tilde{\omega}_{i}^{\prime-1}} E_{\alpha_{i}}^{\left(\lambda_{i}\right)} u_{-\lambda}\right) \\
& =q^{M_{+}}(-1)^{M_{+}^{\vee}} q^{-N_{+}}(-1)^{N_{+}^{\vee}} T_{\tilde{\omega}_{i}^{\prime}}\left(E_{\alpha_{i}}^{\left(\lambda_{i}\right)}\right) S_{\tilde{\omega}_{i}^{\prime}} S_{\tilde{\omega}_{i}^{\prime-1}} E_{\alpha_{i}}^{\left(\lambda_{i}\right)} u_{-\lambda} \\
& =q^{M_{+}}(-1)^{M_{+}^{\vee}} q^{-N_{+}}(-1)^{N_{+}^{\vee}} E_{d_{i} \delta-\alpha_{i}}^{\left(\lambda_{i}\right)} E_{\alpha_{i}}^{\left(\lambda_{i}\right)} u_{-\lambda}
\end{aligned}
$$

by Lemma 2.14. Here

$$
\begin{aligned}
M_{+}=\sum_{\alpha \in \Delta^{+} \cap \tilde{\omega}_{i}^{\prime-1}\left(\Delta^{-}\right)} & \max \left(-\left(\alpha, \tilde{\omega}_{i}^{\prime-1} s_{i}(\lambda)\right), 0\right) \\
& \text { and } N_{+}=\sum_{\alpha \in \Delta^{+} \cap \tilde{\omega}_{i}^{\prime-1}\left(\Delta^{-}\right)} \max \left(-\left(\alpha, s_{i} \tilde{\omega}_{i}^{\prime-1} s_{i}(\lambda)\right), 0\right)
\end{aligned}
$$

and $M_{+}^{\vee}, N_{+}^{\vee}$ are defined by replacing $\alpha$ by $\alpha^{\vee}$. We have used Remark 3.6 in the last equality.

We set $\alpha^{\prime}=-\tilde{\omega}_{i}^{\prime} \alpha$. Then $\alpha^{\prime} \in \Delta^{+} \cap \tilde{\omega}_{i}^{\prime} \Delta^{-}$and

$$
\begin{gathered}
-\left(\alpha, s_{i} \tilde{\omega}_{i}^{\prime-1} s_{i}(\lambda)\right)=\left(\alpha^{\prime}, \tilde{\alpha}_{i} \lambda\right)=\left(\alpha^{\prime}, \lambda\right), \\
-\left(\alpha, \tilde{\omega}_{i}^{\prime-1} s_{i}(\lambda)\right)=\left(\alpha^{\prime}, s_{i} \lambda\right) .
\end{gathered}
$$

For $\beta \in \Delta_{\text {cl }}$, let us denote by $\beta^{\prime}$ the unique element of $\Delta^{+}$such that $\operatorname{cl}\left(\beta^{\prime}\right)=\beta$ and $\beta^{\prime}-n \delta \notin \Delta^{+}$for any $n>0$. We have

$$
\Delta^{+} \cap \tilde{\omega}_{i} \Delta^{-}=\left\{\beta^{\prime}+n d_{\beta} \delta \mid \beta \in \Delta_{\mathrm{cl}}, n \in \mathbf{Z}, 0 \leq n<-\left(\tilde{\omega}_{i}, \beta\right) / d_{\beta}\right\} .
$$

Therefore,

$$
\begin{aligned}
\Delta^{+} \cap \tilde{\omega}_{i}^{\prime}\left(\Delta^{-}\right) & =\Delta^{+} \cap \tilde{\omega}_{i}\left(\Delta^{-}\right) \backslash\left\{\tilde{\omega}_{i}^{\prime}\left(\alpha_{i}\right)\right\} \\
& =\left\{\beta^{\prime}+n d_{\beta} \delta \mid \beta \in \Delta_{\mathrm{cl}}, n \in \mathbf{Z}, 0 \leq n<-\left(\tilde{\omega}_{i}, \beta\right) / d_{\beta}\right\} \backslash\left\{d_{i} \delta-\alpha_{i}\right\},
\end{aligned}
$$

where we have used $\left(\tilde{\omega}_{i}, \operatorname{cl}\left(\alpha_{i}\right)\right)=d_{i}$. If $\beta \in \Delta_{\text {cl }}^{+}$, then $\left(\tilde{\omega}_{i}, \beta\right) \geq 0$ and there are no corresponding terms in the summation of $M_{+}, N_{+}$. If $\beta \in \Delta_{\text {cl }}^{-}$, then $\left(\alpha^{\prime}, \lambda\right) \leq 0$ for $\alpha^{\prime}=\beta^{\prime}+n d_{\beta} \delta$ and $\left(\alpha^{\prime}, s_{i} \lambda\right) \leq 0$ except possibly when $\beta=-\operatorname{cl}\left(\alpha_{i}\right)$, i.e., $\alpha^{\prime}=-\alpha_{i}+n d_{i} \delta$. However, there are no such roots in $\Delta^{+} \cap \tilde{\omega}_{i}^{\prime}\left(\Delta^{-}\right)$. Therefore $M_{+}=N_{+}=0$. By the same reason, we also have $M_{+}^{\vee}=N_{+}^{\vee}=0$.

Next consider the case $\left(X_{N}^{(r)}, i\right)=\left(A_{2 n}^{(2)}, n\right)$. We have an identity $\tilde{\alpha}_{n}=s_{\delta-2 \alpha_{n}} s_{n}$. Following [12, §4.2], we set

$$
w=s_{0} s_{1} \ldots s_{n} \in \hat{W} .
$$

(Our numbering is different from one in [loc. cit.]) We have $w^{n-1}\left(\alpha_{0}\right)=\delta-2 \alpha_{n}$. We can repeat the above calculation replacing the identity by $\tilde{\alpha}_{n}=w^{n-1} s_{0} w^{1-n} s_{n}$. We have

$$
S_{\tilde{\alpha}_{n}} u_{-\lambda}=q^{M_{+}}(-1)^{M_{+}^{\vee}} q^{-N_{+}}(-1)^{N_{+}^{\vee}} T_{w^{n-1}}\left(E_{\alpha_{0}}^{\left(\lambda_{n}\right)}\right) E_{\alpha_{n}}^{\left(2 \lambda_{n}\right)} u_{-\lambda},
$$

where

$$
\begin{aligned}
& M_{+}=\sum_{\alpha^{\prime} \in \Delta^{+} \cap w^{n-1}\left(\Delta^{-}\right)} \max \left(\left(\alpha^{\prime}, s_{n}(\lambda)\right), 0\right), \\
& N_{+}=\sum_{\alpha^{\prime} \in \Delta^{+} \cap w^{n-1}\left(\Delta^{-}\right)} \max \left(\left(\alpha^{\prime}, \lambda\right), 0\right),
\end{aligned}
$$

and $N_{+}^{\vee}, M_{+}^{\vee}$ are similar. 
We have $\Delta^{+} \cap w^{n-1}\left(\Delta^{-}\right) \cap \mathrm{cl}^{-1}\left(\Delta_{\mathrm{cl}}^{+}\right)=\emptyset$. Therefore the above summations are over $\alpha^{\prime} \in \Delta^{+} \cap w^{n-1}\left(\Delta^{-}\right) \cap \mathrm{cl}^{-1}\left(\Delta_{\mathrm{cl}}^{-}\right)$. Then $\left(\alpha^{\prime}, \lambda\right) \leq 0$ for any such $\alpha^{\prime}$, and $\left(\alpha^{\prime}, s_{n}(\lambda)\right) \leq 0$ except possibly when $\operatorname{cl}\left(\alpha^{\prime}\right)=-\operatorname{cl}\left(\alpha_{n}\right)$ or $-2 \operatorname{cl}\left(\alpha_{n}\right)$, i.e., $\alpha^{\prime}=$ $m \delta-\alpha_{n}$ or $(2 m-1) \delta-2 \alpha_{n}$ for some $m \in \mathbf{Z}_{>0}$. However we have

$$
w^{1-n}\left(\alpha_{n}\right)=\alpha_{1}+\cdots+\alpha_{n}
$$

by [12, 4.2.3], which means that such $\alpha^{\prime}$ cannot be in $\Delta^{+} \cap w^{n-1}\left(\Delta^{-}\right)$. Therefore $M_{+}=M_{+}^{\vee}=N_{+}=N_{+}^{\vee}=0$.

Finally we have $T_{w^{n-1}}\left(E_{\alpha_{0}}\right)=E_{\delta-2 \alpha_{n}}$ by [12, 4.2.6].

Let $z_{i}$ be the $\mathbf{U}^{\prime}$-module automorphism of $V\left(\varpi_{i}\right)$ defined in $\S 2.6$.

Lemma 4.6. Let $i \in I_{0}$. Then on $V\left(\varpi_{i}\right)$ :

$$
\tilde{P}_{i,-d_{i}} u_{\varpi_{i}}=\overline{\tilde{P}_{i,-d_{i}} u_{\varpi_{i}}}=z_{i}^{-1} u_{\varpi_{i}}, \quad \tilde{P}_{i,-k d_{i}} u_{\varpi_{i}}=0, \quad \text { for } k>1 .
$$

Proof. The statement for $k>1$ was already observed in the proof of Proposition 4.3(ii). Lemma 4.5 (with $\lambda=\varpi_{i}, \lambda_{i}=1$ ) says $\tilde{P}_{i,-d_{i}} u_{\varpi_{i}}=S_{\tilde{\alpha}_{i}} u_{\varpi_{i}}$. In particular, $\tilde{P}_{i,-d_{i}} u_{\varpi_{i}}$ is an element of the global basis. On the other hand, $V\left(\varpi_{i}\right)$ has a unique global basis element of weight $\varpi_{i}-d_{i} \delta$ (see Theorem 2.16), which by definition equals $S_{w} u_{\varpi_{i}}=z_{i}^{-1} u_{\varpi_{i}}$, where $w=\tilde{\alpha}_{i}$. The assertion follows.

4.2. The map $\Phi_{\lambda}$ and Kashiwara's conjecture. Let $\lambda=\sum_{i \in I_{0}} \lambda_{i} \varpi_{i} \in P_{+}^{0}$. The module

$$
\tilde{V}(\lambda)=\bigotimes_{i \in I_{0}} V\left(\varpi_{i}\right)^{\otimes \lambda_{i}}
$$

has a crystal base $\left(\bigotimes_{i} \mathcal{L}\left(\varpi_{i}\right)^{\otimes \lambda_{i}}, \bigotimes_{i} \mathcal{B}\left(\varpi_{i}\right)^{\otimes \lambda_{i}}\right)$. Let $\tilde{u}_{\lambda}=\bigotimes_{i} u_{\varpi_{i}}^{\otimes \lambda_{i}}$

For each $i$, and each of the $\nu=1, \ldots, \lambda_{i}$ factors of $V\left(\varpi_{i}\right)^{\otimes \lambda_{i}}$, we let $z_{i, \nu}$ be the commuting automorphisms defined in [18, §4.2]. By [18, Theorem 8.5], the submodule

$$
\breve{V}(\lambda)={ }_{\mathcal{A}} \mathbf{U}\left[z_{i, \nu}^{ \pm 1}\right]_{1 \leq i \leq n, 1 \leq \nu \leq \lambda_{i}} \tilde{u}_{\lambda} \subset \tilde{V}(\lambda)
$$

has a global crystal basis $(\breve{\mathcal{L}}, \breve{\mathcal{B}})$ such that $\breve{\mathcal{L}} \subset \bigotimes_{i} \mathcal{L}\left(\varpi_{i}\right)^{\otimes \lambda_{i}}, \breve{\mathcal{B}}=\bigotimes_{i} \mathcal{B}\left(\varpi_{i}\right)^{\otimes \lambda_{i}}$. Since $\breve{V}(\lambda)$ contains the extremal vector $\tilde{u}_{\lambda}$ of weight $\lambda$ we have a unique $\mathbf{U}$-linear morphism

$$
\Phi_{\lambda}: V(\lambda) \rightarrow \breve{V}(\lambda)
$$

sending $u_{\lambda}$ to $\tilde{u}_{\lambda}$, and which commutes with the crystal operators $\tilde{e}_{i}, \tilde{f}_{i}$.

For each $n$-tuple of partitions $\mathbf{c}_{0}=\left(\rho^{(1)}, \rho^{(2)}, \ldots, \rho^{(n)}\right)$ we consider the product of Schur functions in the variables $z_{i, \nu}^{ \pm 1}$ (see [26]):

$$
s_{\mathbf{c}_{0}}\left(z_{i, \nu}^{ \pm 1}\right)=\prod_{i \in I_{0}} s_{\rho^{(i)}}\left(z_{i, 1}^{ \pm 1}, \ldots, z_{i, \lambda_{i}}^{ \pm 1}\right) .
$$

Note that for each $i, s_{\rho^{(i)}}\left(z_{i, \nu}^{ \pm 1}\right)$ acts as the 0 map if $\lambda_{i}<\ell\left(\rho^{(i)}\right)$. We will omit the indices $i, \nu$ and write $s_{\mathbf{c}_{0}}\left(z^{ \pm 1}\right)$.

Using Lemma 4.6 we have:

Proposition 4.10. Let $\mathbf{c}_{0}=\left(\rho^{(1)}, \rho^{(2)}, \ldots, \rho^{(n)}\right)$ be an $n$-tuple of partitions:

$$
\Phi_{\lambda}\left(S_{\mathbf{c}_{0}}^{-} u_{\lambda}\right)=s_{\mathbf{c}_{0}}\left(z^{-1}\right) \tilde{u}_{\lambda} .
$$


Proof. Note that $\sigma \circ\left({ }^{\vee} x^{\vee}\right) \circ \Delta(a)=\Delta\left(a^{\vee}\right)$ for $a \in \mathbf{U}$. Here $\sigma$ is the exchange of two factors of the tensor product. Since our $\tilde{P}_{i,-k d_{i}}(k>0)$ are those given in Sect. 3 after applying $-\circ \vee$ we have by Proposition 3.22 and [23, 3.1.5] (after noting the difference between our coproduct and the one there)

$$
\begin{aligned}
\Delta\left(\tilde{P}_{i,-k d_{i}}\right)=\sum_{s=0}^{k} \tilde{P}_{i,-s d_{i}} \otimes & \tilde{P}_{i,(s-k) d_{i}} \\
& + \text { terms acting as } 0 \text { on } v_{\varpi_{j_{1}}} \otimes v_{\varpi_{j_{2}}} \text { for } j_{1}, j_{2} \in I_{0} .
\end{aligned}
$$

This and Lemma 4.6 imply that $\Delta^{\lambda_{i}}\left(\tilde{P}_{i,-k d_{i}}\right)$ acts as $e_{k}\left(z_{i, 1}^{-1}, \ldots, z_{i, \lambda_{i}}^{-1}\right)$ on $\tilde{V}(\lambda)$ where $e_{k}$ is the $k$-th elementary symmetric function. Since polynomials in the $\tilde{P}_{i,-k d_{i}}$ (resp. elementary symmetric functions) generate the Schur functions $S_{\mathbf{c}_{0}}^{-}$ (resp. $s_{\mathbf{c}_{0}}\left(z^{-1}\right)$ ), we have $\Phi_{\lambda}\left(S_{\mathbf{c}_{0}}^{-} u_{\lambda}\right)=s_{\mathbf{c}_{0}}\left(z^{-1}\right) \tilde{u}_{\lambda}$.

Next we consider the image of $\mathcal{B}(\lambda)$ under $\Phi_{\lambda}$. By Proposition 4.3 every element of $\mathcal{B}(\lambda)$ is connected to an extremal vector of the form $\operatorname{sgn}\left(\mathbf{c}_{0}, 0\right) S_{\mathbf{c}_{0}}^{-} u_{\infty} \otimes t_{\lambda} \otimes$ $u_{-\infty} \bmod q_{s} \mathcal{L}(\lambda)$. Therefore we have,

$$
\begin{aligned}
\mathcal{B}(\lambda)=\left\{X_{1} X_{2} \cdots X_{n}\left(\operatorname{sgn}\left(\mathbf{c}_{0}, 0\right) S_{\mathbf{c}_{0}}^{-} u_{\lambda} \bmod q_{s} \mathcal{L}(\lambda)\right) \mid\right. \\
\left.X_{i}=\tilde{e}_{i} \text { or } \tilde{f}_{i}, \mathbf{c}_{0} \in \mathbf{N}^{\mathcal{R}_{0}}(\lambda)\right\} \backslash\{0\} .
\end{aligned}
$$

Since $\Phi_{\lambda}$ commutes with crystal operators, and the $z_{i, \nu}$ induce automorphisms of the $\mathbf{U}^{\prime}$-crystal of $V\left(\varpi_{i}\right)$, we have $\Phi_{\lambda}(\mathcal{L}(\lambda)) \subset \breve{\mathcal{L}}$ by Proposition 4.10 . Denote by $\Phi_{\lambda \mid q=0}$ the induced map $\mathcal{L}(\lambda) / q_{s} \mathcal{L}(\lambda) \rightarrow \breve{\mathcal{L}} / q_{s} \breve{\mathcal{L}}$.

Proposition 4.13. Let $\mathcal{B}_{0}(\breve{V}(\lambda))$ be the connected component of $\breve{\mathcal{B}}$ containing $\tilde{u}_{\lambda}$. Then

$$
\Phi_{\lambda \mid q=0}:\{b \mid b \in \mathcal{B}(\lambda)\} \rightarrow\left\{\operatorname{sgn}\left(\mathbf{c}_{0}, 0\right) s_{\mathbf{c}_{0}}\left(z^{-1}\right) b^{\prime} \mid \mathbf{c}_{0} \in \mathbf{N}^{\mathcal{R}_{0}}(\lambda), b^{\prime} \in \mathcal{B}_{0}(\breve{V}(\lambda))\right\}
$$

is a bijection.

Proof. It is clear that $\Phi_{\lambda \mid q=0}(\mathcal{B}(\lambda)) \backslash\{0\}$ is equal to the right hand side of the above. Using (4.12), we check that $\Phi_{\lambda \mid q=0}(\mathcal{B}(\lambda))$ does not contain 0 and is injective. Let $b \in \mathcal{B}(\lambda)$ such that $\Phi_{\lambda \mid q=0}(b)=0$. Since $b$ is connected by crystal operators to $b_{1} \otimes t_{\lambda} \otimes u_{-\infty}$, where $b_{1}=\operatorname{sgn}\left(\mathbf{c}_{0}, 0\right) S_{\mathbf{c}_{0}}^{-} u_{\infty} \bmod q_{s} \mathcal{L}(\infty), \mathbf{c}_{0} \in \mathbf{N}^{\mathcal{R}_{0}}(\lambda)$, this implies $\Phi_{\lambda \mid q=0}\left(S_{\mathbf{c}_{0}}^{-} u_{\lambda} \bmod q_{s} \mathcal{L}(\lambda)\right)=0$. This contradicts Proposition 4.10. Now let $b_{1}, b_{2} \in \mathcal{B}(\lambda), b_{1} \neq b_{2}$ and assume $\Phi_{\lambda \mid q=0}\left(b_{1}\right)=\Phi_{\lambda \mid q=0}\left(b_{2}\right)$. By applying crystal operators we may assume $b_{1}=\operatorname{sgn}\left(\mathbf{c}_{0}, 0\right) S_{\mathbf{c}_{0}}^{-} u_{\lambda} \bmod q_{s} \mathcal{L}(\lambda)$ is extremal and purely imaginary, and where $b_{2}$ is of the same weight as $b_{1}$. For $w \in W$, we have

$$
\left.\Phi_{\lambda}\right|_{q=0}\left(X_{i} S_{w} b_{1}\right)=\left.X_{i} S_{w} \Phi_{\lambda}\right|_{q=0}\left(b_{1}\right)=\left.X_{i} S_{w} \Phi_{\lambda}\right|_{q=0}\left(b_{2}\right)=\left.\Phi_{\lambda}\right|_{q=0}\left(X_{i} S_{w} b_{2}\right)=0,
$$

where $X_{i}$ is $\tilde{e}_{i}$ or $\tilde{f}_{i}$. Since $\left.\operatorname{Ker} \Phi_{\lambda}\right|_{q=0} \cap \mathcal{B}(\lambda)=\emptyset$, we have $X_{i} S_{w} b_{2}=0$. Therefore, $b_{2}$ is also extremal. Applying a sequence of $\tilde{f}_{i}^{\max }$ 's (since $b_{2}$ is extremal, this is equivalent to the Weyl group action) we may assume $b_{2}=\operatorname{sgn}\left(\mathbf{c}^{\prime}, 0\right) S_{\mathbf{c}_{0}^{\prime}}^{-} u_{\lambda} \bmod$ $q_{s} \mathcal{L}(\lambda)$ (as in 18, Theorem 5.1]). Then we have that $\Phi_{\lambda}\left(b_{1}\right)=\Phi_{\lambda}\left(b_{2}\right) \neq 0$ and also that $b_{1}=\operatorname{sgn}\left(\mathbf{c}^{\prime \prime}, 0\right) S_{\mathbf{c}_{0}^{\prime \prime}}^{-} u_{\lambda} \bmod q_{s} \mathcal{L}(\lambda)$ for some $\mathbf{c}_{0}^{\prime \prime}$ purely imaginary in $\mathcal{C}$ by Proposition 4.3 (ii). But by Proposition 4.10 it is clear that $\Phi_{\lambda}\left(\operatorname{sgn}\left(\mathbf{c}_{0}^{\prime}, 0\right) S_{\mathbf{c}_{0}^{\prime}}^{-} u_{\lambda}\right)=$ $\Phi_{\lambda}\left(\operatorname{sgn}\left(\mathbf{c}_{0}^{\prime \prime}, 0\right) S_{\mathbf{c}_{0}^{\prime \prime}}^{-} u_{\lambda}\right)$ only if $S_{\mathbf{c}_{0}^{\prime}}^{-} u_{\lambda}=S_{\mathbf{c}_{0}^{\prime \prime}}^{-} u_{\lambda}$. 
Lemma 4.14. (i) Let $\mathbf{c}_{0}, \mathbf{c}_{0}^{\prime} \in \mathbf{N}^{\mathcal{R}_{0}}(\lambda)$ and $b, b^{\prime} \in \mathcal{B}_{0}(\breve{V}(\lambda))$. Then $s_{\mathbf{c}_{0}}\left(z^{-1}\right) b=$ $s_{\mathbf{c}_{0}^{\prime}}\left(z^{-1}\right) b^{\prime}$ if and only if $s_{\mathbf{c}_{0}^{\prime}}\left(z^{-1}\right)=s_{\mathbf{c}_{0}}\left(z^{-1}\right) p(z)$ and $b^{\prime}=p(z)^{-1} b$ for some $p(z)=$ $\prod_{i}\left(z_{i, 1} \cdots z_{i, \lambda_{i}}\right)^{r_{i}}\left(r_{i} \in \mathbf{Z}\right)$.

(ii) For $b \in \mathcal{B}_{0}(\breve{V}(\lambda))$ and $p(z)$ as above, we have $p(z) b \in \mathcal{B}_{0}(\breve{V}(\lambda))$.

Proof. (i) Recall that for fundamental representations $V\left(\varpi_{i}\right)$, we have an isomorphism $V\left(\varpi_{i}\right) \cong \mathbf{Q}\left(q_{s}\right)\left[z_{i}^{ \pm}\right] \otimes W\left(\varpi_{i}\right)$, where $W\left(\varpi_{i}\right)$ is the finite dimensional representation (see $\$ 2.8$ ). For general $\lambda=\sum_{i} \lambda_{i} \varpi_{i} \in P_{+}^{0}$, we put

$$
W(\lambda)=\bigotimes_{i \in I_{0}} W\left(\varpi_{i}\right)^{\otimes \lambda_{i}}
$$

This is irreducible and has a global crystal basis $\mathcal{B}_{W}(\lambda)$. We have

$$
\begin{aligned}
& \tilde{V}(\lambda) \cong \mathbf{Q}\left(q_{s}\right)\left[z_{i, \nu}^{ \pm}\right]_{1 \leq i \leq n, 1 \leq \nu \leq \lambda_{i}} \otimes W(\lambda), \\
& \breve{\mathcal{B}} \cong\left\{\text { monomials in } z_{i, \nu}^{ \pm}\right\} \times \mathcal{B}_{W}(\lambda) .
\end{aligned}
$$

Therefore

$$
s_{\mathbf{c}_{0}}\left(z^{-1}\right) b=s_{\mathbf{c}_{0}}\left(z^{-1}\right) m(z) b_{W}, \quad s_{\mathbf{c}_{0}^{\prime}}\left(z^{-1}\right) b^{\prime}=s_{\mathbf{c}_{0}^{\prime}}\left(z^{-1}\right) m^{\prime}(z) b_{W}^{\prime},
$$

for some monomials $m, m^{\prime}$ and $b_{W}, b_{W}^{\prime} \in \mathcal{B}_{W}(\lambda)$. These two are equal if and only if $b_{W}=b_{W}^{\prime}$ and $s_{\mathbf{c}_{0}}\left(z^{-1}\right) m(z)=s_{\mathbf{c}_{0}^{\prime}}\left(z^{-1}\right) m^{\prime}(z)$. Then $p(z)=m(z) / m^{\prime}(z)$ is a symmetric function which is also a monomial. Therefore it must be of the above form.

(ii) Since $p(z)$ commutes with $\tilde{e}_{i}, \tilde{f}_{i}$, we may assume $b=\tilde{u}_{\lambda}$. For $w \in \hat{W}$, let $S_{w}$ denote the corresponding crystal operator. Then we have

$$
S_{w} \tilde{u}_{\lambda}=\bigotimes_{i}\left(S_{w} u_{\varpi_{i}}\right)^{\otimes \lambda_{i}}
$$

by [2. Lemma 1.6] since $\mathcal{B}\left(V\left(\varpi_{i}\right)\right)$ is the affinization of a simple crystal. Let us take $w=t\left(-\sum_{i} r_{i} \tilde{\alpha}_{i}\right)$. By Lemmas 4.5, 4.6 we get

$$
S_{w} \tilde{u}_{\lambda}=\bigotimes_{i, \nu}\left(z_{i, \nu}\right)^{r_{i}} \tilde{u}_{\lambda}=p(z) \tilde{u}_{\lambda} .
$$

Thus $p(z) \tilde{u}_{\lambda}$ is connected to $\tilde{u}_{\lambda}$ in the crystal graph.

Let

$$
\mathbf{N}^{\mathcal{R}_{0}}(\lambda)^{\prime}=\left\{\left(\rho^{(1)}, \ldots, \rho^{(n)}\right) \mid \rho^{(i)} \text { a partition, } \ell\left(\rho^{(i)}\right)<\lambda_{i}, i=1, \ldots, n\right\} .
$$

This can be identified with the set of irreducible representations of $\prod_{i} S L_{\lambda_{i}}(\mathbf{C})$. Any $\mathbf{c}_{0} \in \mathbf{N}^{\mathcal{R}_{0}}(\lambda)$ decomposes uniquely as $s_{\mathbf{c}_{0}}\left(z^{-1}\right)=s_{\mathbf{c}_{0}^{\prime}}\left(z^{-1}\right) p(z)$ with $\mathbf{c}_{0}^{\prime} \in$ $\mathbf{N}^{\mathcal{R}_{0}}(\lambda)^{\prime}$ and a monomial $p(z)$ as in the above lemma. Therefore Proposition 4.13 can be strengthened as

$$
\begin{aligned}
\Phi_{\lambda \mid q=0}: \mathcal{B}(\lambda) \stackrel{\sim}{\rightarrow}\{ & \left\{\operatorname{sgn}\left(\mathbf{c}_{0}, 0\right) s_{\mathbf{c}_{0}}\left(z^{-1}\right) b^{\prime} \mid \mathbf{c}_{0} \in \mathbf{N}^{\mathcal{R}_{0}}(\lambda)^{\prime}, b^{\prime} \in \mathcal{B}_{0}(\breve{V}(\lambda))\right\} \\
& \cong \mathbf{N}^{\mathcal{R}_{0}}(\lambda)^{\prime} \times \mathcal{B}_{0}(\breve{V}(\lambda)) .
\end{aligned}
$$

A connected component of $\mathcal{B}(\lambda)$ is mapped to $\left\{\mathbf{c}_{0}\right\} \times \mathcal{B}_{0}(\breve{V}(\lambda))$ for some $\mathbf{c}_{0} \in$ $\mathbf{N}^{\mathcal{R}_{0}}(\lambda)^{\prime}$. In particular, each connected component is isomorphic to each other as a $P_{\mathrm{cl}}$-crystal.

Corollary 4.15. The map $\Phi_{\lambda}: V(\lambda) \rightarrow \breve{V}(\lambda)$ is injective. 
Proof. Since $\Phi_{\lambda \mid q=0}: \mathcal{L}(\lambda) / q_{s} \mathcal{L}(\lambda) \rightarrow \breve{\mathcal{L}} / q_{s} \breve{\mathcal{L}}$ maps the crystal basis $\mathcal{B}(\lambda)$ bijectively to $\left\{\operatorname{sgn}\left(\mathbf{c}_{0}, 0\right) s_{\mathbf{c}_{0}}\left(z^{-1}\right) b^{\prime} \mid \mathbf{c}_{0} \in \mathbf{N}^{\mathcal{R}_{0}}(\lambda)^{\prime}, b^{\prime} \in \mathcal{B}_{0}(\breve{V}(\lambda))\right\}$, which is linearly independent. Therefore $\Phi_{\lambda \mid q=0}$ is injective. Write an element $v \in \operatorname{ker} \Phi_{\lambda}, v \neq 0$, in terms of the global basis $\{G(b) \mid b \in \mathcal{B}(\lambda)\}$ as $v=\sum_{b} c_{b}\left(q_{s}\right) G(b)$. Multiplying by a power of $q$ we may assume that each $c_{b}\left(q_{s}\right)$ is regular at $q=0$, so that $v \bmod q_{s} \mathcal{L}(\lambda) \neq 0$. This implies $\Phi_{\lambda \mid q=0}\left(v \bmod q_{s} \mathcal{L}(\lambda)\right) \neq 0$, which is a contradiction.

We now state a main result in this subsection:

Theorem 4.16. (i) We have an isomorphism of crystals

$$
\begin{aligned}
\Phi_{\lambda \mid q=0}: \mathcal{B}(\lambda) \stackrel{\sim}{\rightarrow}\{ & \left\{\operatorname{sgn}\left(\mathbf{c}_{0}, 0\right) s_{\mathbf{c}_{0}}\left(z^{-1}\right) b^{\prime} \mid \mathbf{c}_{0} \in \mathbf{N}^{\mathcal{R}_{0}}(\lambda)^{\prime}, b^{\prime} \in \mathcal{B}_{0}(\breve{V}(\lambda))\right\} \\
& \cong \mathbf{N}^{\mathcal{R}_{0}}(\lambda)^{\prime} \times \mathcal{B}_{0}(\breve{V}(\lambda)) .
\end{aligned}
$$

A connected component of $\mathcal{B}(\lambda)$ is mapped to $\left\{\mathbf{c}_{0}\right\} \times \mathcal{B}_{0}(\breve{V}(\lambda))$ for some $\mathbf{c}_{0} \in$ $\mathbf{N}^{\mathcal{R}_{0}}(\lambda)^{\prime}$. Also, any two connected components are isomorphic to each other as $P_{\mathrm{cl}-\text { crystals. }}$

(ii) $\Phi_{\lambda}$ induces a bijection between the sets

$$
\Phi_{\lambda}: G(\mathcal{B}(\lambda)) \rightarrow\left\{\operatorname{sgn}\left(\mathbf{c}_{0}, 0\right) s_{\mathbf{c}_{0}}\left(z^{-1}\right) G\left(b^{\prime}\right) \mid \mathbf{c}_{0} \in \mathbf{N}^{\mathcal{R}_{0}}(\lambda)^{\prime}, b^{\prime} \in \mathcal{B}_{0}(\breve{V}(\lambda))\right\} .
$$

(iii) $A$ vector $b \in \mathcal{B}(\lambda)$ is extremal if and only if

$$
\Phi_{\lambda \mid q=0}(b)=\operatorname{sgn}\left(\mathbf{c}_{0}, 0\right) s_{\mathbf{c}_{0}}\left(z^{-1}\right) S_{w} \tilde{u}_{\lambda}
$$

for some $\mathbf{c}_{0} \in \mathbf{N}^{\mathcal{R}_{0}}(\lambda)^{\prime}, w \in \hat{W}$.

Proof. (i) is proved already. Let us show (ii). By Proposition 4.13, for each $b \in \mathcal{B}(\lambda)$ there exist $b^{\prime} \in \mathcal{B}_{0}(\breve{V}(\lambda))$ and $s_{\mathbf{c}_{0}}\left(z^{-1}\right)$ such that $\Phi_{\lambda}(b)=s_{\mathbf{c}_{0}}\left(z^{-1}\right) b^{\prime} \bmod q_{s} \breve{\mathcal{L}}$. Let $G(b), G\left(b^{\prime}\right)$ be the respective globalizations of $b$ and $b^{\prime}$. Then $\Phi_{\lambda}(G(b)) \equiv$ $s_{\mathbf{c}_{0}}\left(z^{-1}\right) G\left(b^{\prime}\right) \bmod q_{s} \breve{\mathcal{L}}$. Since $\Phi_{\lambda}$ commutes with the bar involution, we have $\Phi_{\lambda}(G(b)) \equiv s_{\mathbf{c}_{0}}\left(z^{-1}\right) G\left(b^{\prime}\right) \bmod q_{s}^{-1} \overline{\mathcal{L}}$. We get the assertion.

Let us prove (iii). It is enough to consider the case when $\Phi_{\lambda \mid q=0}(b) \in \mathcal{B}_{0}(\breve{V}(\lambda))$. As in the proof of Lemma 4.14, we can write $\Phi_{\lambda \mid q=0}(b)=p(z) b_{W}$ where $p(z)=$ $\prod_{i}\left(z_{i, 1} \cdots z_{i, \lambda_{i}}\right)^{r_{i}}\left(r_{i} \in \mathbf{Z}\right)$ and $b_{W} \in \mathcal{B}_{W}(\lambda)$. Then $b$ is extremal if and only if $b_{W}$ is so. Furthermore, $b_{W}$ is extremal if and only if $b_{W}=S_{w_{0}} \tilde{u}_{\lambda}$ for $w_{0} \in W_{\mathrm{cl}}$. This follows from [2, Lemma 1.6], after noting that ([18, Theorem 5.15]) $W\left(\varpi_{i}\right)$ has a simple crystal such that the weight of any extremal vector belongs to $\hat{W} \operatorname{cl}\left(\varpi_{i}\right)$. Now we have $\Phi_{\lambda \mid q=0}(b)=p(z) b_{W}=S_{w} \tilde{u}_{\lambda}$ for some $w \in \hat{W}$ as in the proof of Lemma 4.14.

Remark 4.17. Taken together the results of this section and $\operatorname{sgn}\left(\mathbf{c}_{0}, 0\right)=1$, which will be proved in the next section, give the conjectures [18, 13.1, 13.2]. To obtain 13.1 (iii), consider that the crystal $\bigotimes_{i \in I_{0}} \mathcal{B}\left(\lambda_{i} \varpi_{i}\right)$ is by Proposition 4.13 in bijective correspondence with $\left\{s_{\mathbf{c}_{0}}\left(z^{-1}\right) \mathcal{B}_{0}(\breve{V}(\lambda))\right\}$, and note that $\Phi_{\lambda}$ factors through $\bigotimes_{i \in I_{0}} V\left(\lambda_{i} \varpi_{i}\right)$.

4.3. A Peter-Weyl type decomposition theorem. Let $\mathcal{B}_{0}(\lambda)$ be the connected component of $\mathcal{B}(\lambda)$ containing $u_{\lambda}$. Consider $\bigsqcup_{\lambda \in P} \mathcal{B}_{0}(\lambda) \times \mathcal{B}(-\lambda)$ as a crystal over $\mathfrak{g} \oplus \mathfrak{g}$. Here for $u \otimes v \in \mathcal{B}_{0}(\lambda) \otimes \mathcal{B}(-\lambda), X_{i}(u \otimes v)=X_{i} u \otimes v$ and $X_{i}^{*}(u \otimes v)=$ $u \otimes X_{i} v$ where $\left(X_{i}=\tilde{e}_{i}, \tilde{f}_{i}\right)$. The Weyl group $\hat{W}$ acts on $\bigsqcup_{\lambda \in P} \mathcal{B}_{0}(\lambda) \times \mathcal{B}(-\lambda)$ by $S_{w}^{*} \times S_{w}^{*}: \mathcal{B}_{0}(\lambda) \times \mathcal{B}(-\lambda) \rightarrow \mathcal{B}_{0}(w \lambda) \times \mathcal{B}(-w \lambda)$. 
Consider the map $\mathcal{B}_{0}(\lambda) \times \mathcal{B}(-\lambda) \rightarrow \mathcal{B}\left(\mathbf{U} a_{\lambda}\right)$, which sends $u_{\lambda} \otimes b \in \mathcal{B}_{0}(\lambda) \times \mathcal{B}(-\lambda)$ to $b^{*} \in \mathcal{B}\left(\mathbf{U} a_{\lambda}\right)$. We will show that this is well-defined later. It is a map between crystals over $\mathfrak{g} \oplus \mathfrak{g}$ where the usual crystal structure on $\mathcal{B}(\widetilde{\mathbf{U}})$ corresponds to the one on $\mathcal{B}_{0}(\lambda)$ and the star crystal structure on $\mathcal{B}(\widetilde{\mathbf{U}})$ corresponds to the one on $\mathcal{B}(-\lambda)$. We have

Theorem 4.18. $\left(\bigsqcup_{\lambda \in P} \mathcal{B}_{0}(\lambda) \times \mathcal{B}(-\lambda)\right) / \hat{W} \stackrel{\sim}{\rightarrow} \mathcal{B}(\widetilde{\mathbf{U}})$ as crystals over $\mathfrak{g} \oplus \mathfrak{g}$.

Proof. When $\langle c, \lambda\rangle \neq 0$, the $\mathcal{B}\left(\mathbf{U} a_{\lambda}\right)$ part of the crystal decomposition in Theorem 4.18 appears as 117, Proposition 10.2.2]. Therefore, it is sufficient to check the map in Theorem 4.18 for $\lambda \in P^{0}$, where the image on the right hand side is in $\mathcal{B}\left(\mathbf{U} a_{\lambda}\right)$.

The following proof is a modification of a result of Nakashima [31, Proposition 4.4]. We give it for the sake of the reader.

We first prove

(C1): For any extremal vector $b \in \mathcal{B}(\widetilde{\mathbf{U}})$, there exists a crystal embedding:

$$
\mathcal{B}_{0}(\operatorname{wt}(b)) \hookrightarrow \mathcal{B}(\widetilde{\mathbf{U}}),
$$

given by $u_{\mathrm{wt}(b)} \mapsto b$.

Let $b \in \mathcal{B}(\widetilde{\mathbf{U}})$ be extremal of weight $\lambda$ and $B^{\prime}$ be connected component containing $b$. We want to prove $B^{\prime} \cong \mathcal{B}_{0}($ wt $(b))$. By [17, Corollary 9.3.4] the connected component $B^{\prime}$ of $b$ can be embedded into some $\mathcal{B}(\mu)$ for some $\mu \in P^{0}$. For each $w \in$ $\hat{W}$, we have $S_{w}^{*}(\mathcal{B}(\mu)) \stackrel{\sim}{\rightarrow} \mathcal{B}(w \mu)$. Using this Weyl group action, and noting that for $\operatorname{cl}(\mu)=\operatorname{cl}\left(\mu^{\prime}\right), \mathcal{B}(\mu) \stackrel{\sim}{\rightarrow} \mathcal{B}\left(\mu^{\prime}\right)$ as $P_{\mathrm{cl}}$ crystals, we may assume $\mu=\sum_{i=1}^{n} m_{i} \varpi_{i} \in P_{0}^{+}$. By Theorem 4.16(iii), (i), we have that $\Phi_{\mu}(b)=\operatorname{sgn}\left(\mathbf{c}_{0}, 0\right) s_{\mathbf{c}_{0}}\left(z^{-1}\right) S_{w} \tilde{u}_{\mu}$ for fixed $\mathbf{c}_{0} \in \mathcal{C}, w \in \widehat{W}$, and $B^{\prime}$ is isomorphic to $B_{0}(\mu)$ as a $P_{\mathrm{cl}}-$ crystal, so that $b$ is mapped to $S_{w} u_{\mu}$. This is further isomorphic to $B_{0}(\lambda)$ so that $b$ is mapped to $u_{\lambda}$. But since wt $(b)=$ wt $\left(u_{\lambda}\right)=\lambda$ this is also an isomorphism of $P$-crystals. This completes the proof of $(\mathbf{C} 1)$.

We now define a map $\varphi: \bigsqcup_{\lambda} \mathcal{B}_{0}(\lambda) \times \mathcal{B}(-\lambda) \rightarrow \mathcal{B}(\widetilde{\mathbf{U}})$ by

$$
\varphi\left(X_{1} X_{2} \cdots X_{N} u_{\lambda} \otimes b\right)=X_{1} X_{2} \cdots X_{N} b^{*},
$$

where $X_{j}=\tilde{e}_{i}$ or $\tilde{f}_{i}$. By (C1) this is well-defined, i.e., (a) if $X_{1} X_{2} \cdots X_{N} u_{\lambda}=$ 0 , then $X_{1} X_{2} \cdots X_{N} b^{*}=0$, and (b) if $X_{1} X_{2} \cdots X_{N} u_{\lambda}=X_{1}^{\prime} X_{2}^{\prime} \cdots X_{N^{\prime}}^{\prime} u_{\lambda}$, then $X_{1} X_{2} \cdots X_{N} b^{*}=X_{1}^{\prime} X_{2}^{\prime} \cdots X_{N^{\prime}}^{\prime} b^{*}$. (C1) is applicable since $b^{*}$ is extremal of weight $\lambda$ for $b \in \mathcal{B}(-\lambda)$.

Since $\tilde{e}_{i}^{*}, \tilde{f}_{i}^{*}$ commute with $\tilde{e}_{j}, \tilde{f}_{j}$ on $\mathcal{B}(\widetilde{\mathbf{U}})([17$, Theorem 5.1.1]), $\varphi$ is a morphism of bi-crystal. Since any connected component of $\mathcal{B}(\widetilde{\mathbf{U}})$ contains an extremal vector (17, Corollary 9.3.3]), the map $\varphi$ is surjective.

Finally we show that $\varphi$ becomes injective if we divide $\bigsqcup_{\lambda} \mathcal{B}_{0}(\lambda) \times \mathcal{B}(-\lambda)$ by $\hat{W}$. Suppose $X_{1} X_{2} \cdots X_{N} b^{*}=X_{1}^{\prime} X_{2}^{\prime} \cdots X_{N^{\prime}}^{\prime} b^{\prime *}$ where each $X_{j}, X_{j^{\prime}}^{\prime}=\tilde{e}_{i}$ or $\tilde{f}_{i}$, $b \in \mathcal{B}(-\lambda), b^{\prime} \in \mathcal{B}\left(-\lambda^{\prime}\right)$. Then $b^{*}$ and $b^{*}$ are in the same connected component of $\mathcal{B}(\widetilde{\mathbf{U}})$, which is isomorphic to $\mathcal{B}_{0}(\lambda)$ by $(\mathbf{C 1})$. By Theorem 4.16(iii), we have $b^{\prime *}=S_{w} b^{*}$ for some $w \in \hat{W}$. In particular, $\lambda^{\prime}=w \lambda$. We have $X_{1} X_{2} \cdots X_{N} b^{*}=$ $X_{1}^{\prime} X_{2}^{\prime} \cdots X_{N^{\prime}}^{\prime} S_{w} b^{*}$. (C1) implies $X_{1} X_{2} \cdots X_{N} u_{\lambda}=X_{1}^{\prime} X_{2}^{\prime} \cdots X_{N^{\prime}}^{\prime} S_{w} u_{\lambda}$. The isomorphism $S_{w}^{*}: \mathcal{B}_{0}(\lambda) \rightarrow \mathcal{B}_{0}(w \lambda)$ sends $u_{\lambda}$ to $S_{w}^{-1} u_{w \lambda}=S_{w}^{-1} u_{\lambda^{\prime}}$. Therefore

$$
X_{1}^{\prime} X_{2}^{\prime} \cdots X_{N^{\prime}}^{\prime} u_{\lambda^{\prime}} \otimes b^{\prime}=\left(S_{w}^{*} \times S_{w}^{*}\right)\left(X_{1} X_{2} \cdots X_{N} u_{\lambda} \otimes b\right) .
$$


Remark 4.19. In 31 a condition labeled (C2) requiring $\mathcal{B}_{0}(\lambda)_{\lambda}=\left\{u_{\lambda}\right\}$ is used. This is false in general. A counter-example for type $A_{2}^{(1)}$ can be found in [18, 5.10].

\section{The Proof of Theorem 3.13(II)}

In this section we will complete the proof of Theorem 3.13 . The proof uses results in the previous section, in particular extremal weight modules.

Remark 5.1. Since we are working with generators in $\mathbf{U}^{+}$we will need to consider the extremal weight modules $V(-\lambda), \lambda \in P_{+}^{0}$.

Lemma 5.2. Let $\lambda \in P_{+}^{0}$ and $\mathbf{c}_{0} \in \mathbf{N}^{\mathcal{R}_{0}}(\lambda)$. Then $S_{\mathbf{c}_{0}} u_{-\lambda} \in G(\mathcal{B}(-\lambda))$.

Proof. We have $\operatorname{sgn}\left(\mathbf{c}_{0}, 0\right) S_{\mathbf{c}_{0}} u_{-\lambda} \in G(\mathcal{B}(-\lambda))$ by Proposition 4.10 and Theorem 4.16. So we only need to show $\operatorname{sgn}\left(\mathbf{c}_{0}, 0\right)=1$. Therefore it is enough to show $S_{\mathbf{c}_{0}} u_{-\mu} \in G(\mathcal{B}(-\mu))$ for some $\mu$. We check this by induction on $\sum_{i}\left|\rho^{(i)}\right|$. Take $\mu=\sum_{i} \ell\left(\rho^{(i)}\right) \varpi_{i}$. Fix an $i$ and consider $\rho^{\prime(i)}$ obtained by removing the last box in each row of $\rho^{(i)}$. Let $\mathbf{c}_{0}^{\prime}$ be the set of partitions we get by replacing $\rho^{(i)}$ by $\rho^{\prime(i)}$.

By Lemma 4.5 and the Pieri rules for symmetric functions [26, p. 73],

$$
S_{\mathbf{c}_{0}^{\prime}} S_{\tilde{\alpha}_{i}} u_{-\mu}=S_{\mathbf{c}_{0}^{\prime}} \tilde{P}_{i, \mu_{i} d_{i}} u_{-\mu}=S_{\mathbf{c}_{0}} u_{-\mu}
$$

where $\mu_{i}=\ell\left(\rho^{(i)}\right)$. A priori, the right hand side is a sum over all $S_{\mathbf{c}_{0}} u_{-\mu}$ such that $\mathbf{c}_{0}$ is an $n$-tuple of partitions which are identical to $\mathbf{c}_{0}^{\prime}$ in factors different than $i$, and for $i$ we have $\rho^{(i)} \backslash \rho^{\prime(i)}$ is a vertical $\mu_{i}$ strip. But there is only one such summand. (Under the identification between Schur functions and irreducible representations of $G_{\mu}, \tilde{P}_{i, \mu_{i} d_{i}}$ is identified with a power of the determinant representation. So the tensor product of it with an irreducible representation remains irreducible.)

Let us note the following fact: If $\lambda^{\prime} \in P^{0}$ and $\lambda=\sum_{i} \lambda_{i} \varpi_{i} \in P_{+}^{0}$ satisfy $\operatorname{cl}\left(\lambda^{\prime}\right)=\operatorname{cl}(\lambda)$ then $u_{\infty} \otimes t_{-\lambda} \otimes b_{2} \in \mathcal{B}(\lambda) \Longleftrightarrow u_{\infty} \otimes t_{-\lambda^{\prime}} \otimes b_{2} \in \mathcal{B}\left(\lambda^{\prime}\right)$ (see 18. Appendix]). In other words, $S_{\mathbf{c}_{0}} u_{-\lambda} \bmod q_{s} \mathcal{L}(\lambda) \in \mathcal{B}(\lambda) \Longleftrightarrow S_{\mathbf{c}_{0}} u_{-\lambda^{\prime}} \bmod$ $q_{s} \mathcal{L}\left(\lambda^{\prime}\right) \in \mathcal{B}\left(\lambda^{\prime}\right)$. Then by the inductive assumption, $S_{\mathbf{c}_{0}^{\prime}} u_{\mu_{i} d_{i} \delta-\mu}$ is an element of the global crystal basis. Therefore $S_{\mathbf{c}_{0}} u_{-\mu}=S_{\mathbf{c}_{0}^{\prime}} S_{\tilde{\alpha}_{i}} u_{-\mu}$ is also in the global basis, since the isomorphism $V\left(\mu_{i} d_{i} \delta-\mu\right) \stackrel{\sim}{\rightarrow} V(-\mu)$ sends the global basis to the global basis (see [17, Prop. 8.2.2 (iv)]).

Proof of $\operatorname{sgn}(\mathbf{c}, p)=1$ in Proposition 3.2\%. Let $\mathbf{c}_{0} \in \mathcal{C}$ be purely imaginary. In the proof of Lemma 5.2 we have shown $\operatorname{sgn}\left(\mathbf{c}_{0}, 0\right)=1$. From [25, Proposition 8.3(a)] we have $\operatorname{sgn}(\mathbf{c}, 0)=1$ for an arbitrary $\mathbf{c}$. The general case now follows from 25 . Theorem 1.2] (see $\$ 2.6)$.

Let $b(\mathbf{c}, p)$ as in Proposition 3.27. We denote $G(b(\mathbf{c}, p))$ by $G(\mathbf{c}, p)$. By the same argument in the proof of [23, 42.1.12], we have

$$
\begin{gathered}
\varphi_{i_{p}}(b(\mathbf{c}, p))=\mathbf{c}(p) \quad\left(\text { hence } G(\mathbf{c}, p) \in E_{i_{p}}^{\mathbf{c}(p)} \mathbf{U}^{+}\right), \\
\tilde{e}_{i_{p}}(b(\mathbf{c}, p))=b\left(\mathbf{c}^{+}, p\right), \\
\tilde{f}_{i_{p}}(b(\mathbf{c}, p))=b\left(\mathbf{c}^{-}, p\right) \quad \text { if } \mathbf{c}(p)>0,
\end{gathered}
$$

where $\mathbf{c}^{+}\left(\right.$resp. $\left.\mathbf{c}^{-}\right)$is defined by setting $\mathbf{c}^{+}(p)=\mathbf{c}(p)+1\left(\right.$ resp. $\left.\mathbf{c}^{-}(p)=\mathbf{c}(p)-1\right)$ and other entries are the same as $\mathbf{c}$. We have similar formulas for $\varphi_{i_{p+1}}^{*}, \tilde{f}_{i_{p+1}}^{*}, \tilde{e}_{i_{p+1}}^{*}$.

Let $\mathbf{U}^{+}[i],{ }^{*} \mathbf{U}^{+}[i],{ }^{i} \pi, \pi^{i}$ be as in $\$ 2.6$. By (5.3) we have

$$
{ }^{i_{p}} \pi(G(\mathbf{c}, p)) \neq 0 \Longleftrightarrow \mathbf{c}(p)=0 \Longleftrightarrow \pi^{i_{p}}(G(\mathbf{c}, p-1)) \neq 0,
$$


and

$$
T_{i_{p}}\left({ }^{i_{p}} \pi(G(\mathbf{c}, p))\right)=\pi^{i_{p}}(G(\mathbf{c}, p-1)) .
$$

The latter follows from (3.10) (see 25, $\S 6.2]$ for detail).

Let us define $a_{\mathbf{c}, \mathbf{c}^{\prime}}^{p} \in \mathbf{Q}\left(q_{s}\right)$ by

$$
L(\mathbf{c}, p)=\sum_{\mathbf{c}^{\prime}} a_{\mathbf{c}, \mathbf{c}^{\prime}}^{p} G\left(\mathbf{c}^{\prime}, p\right) .
$$

The sum is over a finite set of $\mathbf{c}^{\prime} \in \mathcal{C}$ indexing all elements $G(\mathbf{c}, p) \in \mathbf{U}^{+}$having the same weight with $L(\mathbf{c}, p)$. Since the global basis is an integral base of ${ }_{\mathcal{A}} \mathbf{U}^{+}$ and $\mathcal{L}(-\infty)$ we have $a_{\mathbf{c}, \mathbf{c}^{\prime}} \in \mathcal{A} \cap \mathbf{A}_{\infty}=\mathbf{Z}\left[q_{s}^{-1}\right]$. We also have $\left.a_{\mathbf{c}, \mathbf{c}^{\prime}}\right|_{q=\infty}=\delta_{\mathbf{c}, \mathbf{c}^{\prime}}$ by Proposition 3.27 together with $\operatorname{sgn}(\mathbf{c}, p)=1$. Theorem 3.13 (ii) follows from

Lemma 5.6. The transition matrix $\left(a_{\mathbf{c}, \mathbf{c}^{\prime}}\right)$ between the global basis and the $L(\mathbf{c}, p)$, $\mathbf{c} \in \mathcal{C}, p \in \mathbf{Z}$ is upper triangular with respect to the ordering $\prec_{p}$, and the diagonal entries are 1.

Proof. We first consider the case $\mathbf{c}_{+}=0=\mathbf{c}_{-}$, so that

$$
L(\mathbf{c}, p)=T_{i_{p+1}} T_{i_{p+2}} \cdots T_{i_{0}}\left(S_{\mathbf{c}_{0}}\right) \quad \text { or } \quad T_{i_{p}}^{-1} T_{i_{p-1}}^{-1} \cdots T_{i_{2}}^{-1} T_{i_{1}}^{-1}\left(S_{\mathbf{c}_{0}}\right) .
$$

In this case, by Lemma 5.2 we know that $L(\mathbf{c}, p) u_{-\lambda}$ is in the global basis of $V(-\lambda)$ for any sufficiently large $\lambda \in P_{+}^{0}$. Therefore $(L(\mathbf{c}, p)-G(\mathbf{c}, p)) u_{-\lambda}=0$ for any sufficiently large $\lambda \in P_{+}^{0}$, i.e.,

$$
\sum_{\mathbf{c}^{\prime} \neq \mathbf{c}}\left(a_{\mathbf{c}, \mathbf{c}^{\prime}}^{p}-\delta_{\mathbf{c}, \mathbf{c}^{\prime}}\right) G\left(\mathbf{c}^{\prime}, p\right) u_{-\lambda}=0 .
$$

By the construction of $V(-\lambda),\left\{G\left(\mathbf{c}^{\prime}, p\right) u_{-\lambda} \mid \mathbf{c}^{\prime} \in \mathcal{C}\right\}$ is mapped to the union of the global basis of $V(-\lambda)$ and 0 . Hence

$$
a_{\mathbf{c}, \mathbf{c}^{\prime}}^{p}=\delta_{\mathbf{c}, \mathbf{c}^{\prime}} \quad \text { or } \quad G\left(\mathbf{c}^{\prime}, p\right) u_{-\lambda}=0 .
$$

If $\mathbf{c}_{+}^{\prime}=0=\mathbf{c}_{-}^{\prime}$, then

$$
G\left(\mathbf{c}^{\prime}, p\right) u_{-\lambda}=T_{i_{p+1}} T_{i_{p+2}} \cdots T_{i_{0}}\left(S_{\mathbf{c}_{0}^{\prime}}\right) u_{-\lambda} \quad \text { or } \quad T_{i_{p}}^{-1} T_{i_{p-1}}^{-1} \ldots T_{i_{2}}^{-1} T_{i_{1}}^{-1}\left(S_{\mathbf{c}_{0}^{\prime}}\right) u_{-\lambda}
$$

is nonzero for sufficiently large $\lambda$. This means that $a_{\mathbf{c}, \mathbf{c}^{\prime}}^{p}=\delta_{\mathbf{c}, \mathbf{c}^{\prime}}$ for such $\mathbf{c}^{\prime}$. By the definition of the ordering, the remaining $\mathbf{c}^{\prime}$ 's have $\mathbf{c}^{\prime} \succ_{p} \mathbf{c}$. We have the assertion in this case.

Next consider the case $\mathbf{c}(p+1)=\mathbf{c}(p+2)=\cdots=0$, i.e.,

$$
\begin{aligned}
L(\mathbf{c}, p)= & \left(E_{i_{p}}^{(\mathbf{c}(p))} T_{i_{p}}^{-1}\left(E_{i_{p-1}}^{(\mathbf{c}(p-1))}\right) T_{i_{p}}^{-1} T_{i_{p-1}}^{-1}\left(E_{i_{p-2}}^{(\mathbf{c}(p-2))}\right) \cdots\right) \\
& \times T_{i_{p+1}} T_{i_{p+2}} \cdots T_{i_{0}}\left(S_{\mathbf{c}_{0}}\right) .
\end{aligned}
$$

We prove the assertion by the induction on $q$ such that $\mathbf{c}(p-q)=\mathbf{c}(p-q-1)=$ $\cdots=0$. When $q=0$, we have $\mathbf{c}_{+}=0=\mathbf{c}_{-}$, which we have checked already.

First assume $\mathbf{c}(p)=0$. We consider $L(\mathbf{c}, p-1)=T_{i_{p}} L(\mathbf{c}, p)$ (see (3.10)). By the induction hypothesis, we have

$$
L(\mathbf{c}, p-1)=G(\mathbf{c}, p-1)+\sum_{\mathbf{c} \prec p-1 \mathbf{c}^{\prime}} a_{\mathbf{c}, \mathbf{c}^{\prime}}^{p-1} G\left(\mathbf{c}^{\prime}, p-1\right) .
$$


We apply the composition $T_{i_{p}}^{-1} \circ \pi^{i_{p}}$ to both sides. We have $L(\mathbf{c}, p-1) \in{ }^{*} \mathbf{U}^{+}\left[i_{p}\right]$, so the left hand side becomes $L(\mathbf{c}, p)$. Therefore

$$
L(\mathbf{c}, p)=T_{i_{p}}^{-1}\left(\pi^{i_{p}}(G(\mathbf{c}, p-1))\right)+\sum_{\mathbf{c} \prec p-1 \mathbf{c}^{\prime}} a_{\mathbf{c}, \mathbf{c}^{\prime}}^{p-1} T_{i_{p}}^{-1}\left(\pi^{i_{p}}\left(G\left(\mathbf{c}^{\prime}, p-1\right)\right)\right) .
$$

By (5.5) the right hand side is contained in

$$
G(\mathbf{c}, p)+\sum_{\substack{\mathbf{c} \prec p-1 \\ \mathbf{c}^{\prime}(p)=0}} a_{\mathbf{c}}^{p-1} \mathbf{c}^{\prime} G\left(\mathbf{c}^{\prime}, p\right)+E_{i_{p}} \mathbf{U}^{+} .
$$

The condition $\mathbf{c}^{\prime}(p)=0$ comes from ${ }^{i_{p}} \pi\left(G\left(\mathbf{c}^{\prime}, p-1\right)\right) \neq 0$ (see (5.4)). The part in $E_{i_{p}} \mathbf{U}^{+}$is a linear combination of $G\left(\mathbf{c}^{\prime \prime}, p\right)$ 's with $\mathbf{c}^{\prime \prime}(p)>0$. Each such $\mathbf{c}^{\prime \prime}$ is greater than $\mathbf{c}$ with respect to $\prec_{p}$. The summation in the second term can be replaced as $\sum_{\mathbf{c} \prec_{p} \mathbf{c}^{\prime}, \mathbf{c}^{\prime}(p)=0}$ by $(3.12)$. Thus we have the assertion under the assumption $\mathbf{c}(p)=0$.

Next we assume $\mathbf{c}(p)>0$. Let us define $\tilde{\mathbf{c}}$ by setting $\tilde{\mathbf{c}}(p)=0$ and all other entries are the same as $\mathbf{c}$. We have

$$
L(\mathbf{c}, p)=E_{i_{p}}^{(\mathbf{c}(p))} L(\tilde{\mathbf{c}}, p) .
$$

Since $\tilde{\mathbf{c}}(p)=0$, we have just proved

$$
L(\tilde{\mathbf{c}}, p)=G(\tilde{\mathbf{c}}, p)+\sum_{\tilde{\tilde{\mathbf{c}}^{\prime} \succ p} \tilde{\mathbf{c}}} a_{\tilde{\mathbf{c}}, \tilde{\mathbf{c}}^{\prime}}^{p} G\left(\tilde{\mathbf{c}}^{\prime}, p\right) .
$$

By [23, 14.3], $E_{i_{p}}^{(\mathbf{c}(p))} G(\tilde{\mathbf{c}}, p)=G(\mathbf{c}, p)$ plus an element in $E_{i_{p}}^{\mathbf{c}(p)+1} \mathbf{U}^{+}$. If we write the element in a linear combination of $G\left(\mathbf{c}^{\prime \prime}, p\right)$ 's, all elements appearing satisfy $\mathbf{c}^{\prime \prime} \succ_{p} \mathbf{c}$ by (5.3). Next consider $E_{i_{p}}^{(\mathbf{c}(p))} G\left(\tilde{\mathbf{c}}^{\prime}, p\right)$, obtained from (5.7) by multiplication by $E_{i_{p}}^{(\mathbf{c}(p))}$. If $\tilde{\mathbf{c}}^{\prime}(p)>0$, then this is an element in $E_{i_{p}}^{\mathbf{c}(p)+1} \mathbf{U}^{+}$. Otherwise, it is equal to $G\left(\mathbf{c}^{\prime}, p\right)$ plus an element in $E_{i_{p}}^{\mathbf{c}(p)+1} \mathbf{U}^{+}$, where $\mathbf{c}^{\prime}$ is defined by setting $\mathbf{c}^{\prime}(p)=\mathbf{c}(p)$ and all other entries are the same as $\tilde{\mathbf{c}}^{\prime}$. In either cases, it is a linear combination of elements $G\left(\mathbf{c}^{\prime \prime}, p\right)$ with $\mathbf{c}^{\prime \prime} \succ_{p} \mathbf{c}$. Thus we have the assertion for $G(\mathbf{c}, p)$.

Finally we consider the general case. We first remark

$$
(\mathbf{c}(p), \mathbf{c}(p-1), \mathbf{c}(p-2), \ldots) \leq\left(\mathbf{c}^{\prime}(p), \mathbf{c}^{\prime}(p-1), \mathbf{c}^{\prime}(p-2), \ldots\right) .
$$

This is proved by the induction on $q$ such that $\mathbf{c}(p-q)=\mathbf{c}(p-q-1)=\cdots=0$. When $q=0$, the left hand side is $(0,0, \ldots)$, so the inequality trivially holds. The remaining argument of the induction is exactly the same as above.

Now we can prove the assertion of the lemma by the induction on $q$ such that $\mathbf{c}(p+q)=\mathbf{c}(p+q+1)=\cdots=0$. When $q=1$, we are reduced to the case studied above, and have the assertion. The remaining argument is almost the same as above. When $\mathbf{c}(p+1)=0$, we apply the induction hypothesis to $L(\mathbf{c}, p+1)$ and consider $T_{i_{p+1}}{ }^{i_{p+1}} \pi L(\mathbf{c}, p+1)$. We use (5.5) to get

$$
L(\mathbf{c}, p) \in G(\mathbf{c}, p)+\sum_{\substack{\mathbf{c} \prec p+1 \\ \mathbf{c}^{\prime}\left(\beta_{p+1}\right)=0}} a_{\mathbf{c}, \mathbf{c}^{\prime}}^{p+1} G\left(\mathbf{c}^{\prime}, p\right)+\mathbf{U}^{+} E_{i_{p+1}} .
$$

The summation in the second part can be replaced as $\sum_{\mathbf{c} \prec_{p} \mathbf{c}^{\prime}, \mathbf{c}^{\prime}(p+1)=0}$ by (3.12). The part in $\mathbf{U}^{+} E_{i_{p+1}}$ is a linear combination of $G\left(\mathbf{c}^{\prime \prime}, p\right)$ 's with $\mathbf{c}^{\prime \prime}(p+1)>0$. Since we already have (5.8) (with $\mathbf{c}^{\prime}$ replaced by $\mathbf{c}^{\prime \prime}$ ), we have $\mathbf{c} \prec_{p} \mathbf{c}^{\prime \prime}$. We have 
the assertion in the case $\mathbf{c}(p+1)=0$. The case $\mathbf{c}(p+1)>0$ can be reduced to the case $\mathbf{c}(p+1)=0$ as above with use of (5.8).

\section{Cell structure of $\widetilde{\mathbf{U}}$.}

In this section we prove Lusztig's conjecture [24] on two-sided cells of the modified quantum affine algebra of level 0 . Our strategy follows the same line as his proof of the corresponding conjecture for finite type cases. However, here we need to show that a certain bi-module $\widetilde{\mathbf{U}}[\lambda]$ has a global crystal basis, whereas for finite type cases this assertion is a direct consequence of the definition. In defining $\widetilde{\mathbf{U}}[\lambda]$, our earlier results on extremal weight modules play a key role. Their role is analogous to the role that dominant highest weight representations play in the finite type case.

Let $\widetilde{\mathbf{U}}$ be the level 0 modified quantum affine algebra, i.e., $\widetilde{\mathbf{U}}=\bigoplus_{\lambda \in P_{\mathrm{cl}}^{0}} \mathbf{U} a_{\lambda}$. Unfortunately this contradicts the notation in Sect. 1 , where $\widetilde{\mathbf{U}}$ was $\bigoplus_{\lambda \in P} \mathbf{U} a_{\lambda}$, but we do not want to introduce new notation. Let $\tilde{\mathcal{L}}$ be its crystal lattice, $\tilde{\mathcal{B}}$ be its crystal base.

Throughout this section, $\lambda$ is a dominant classical level 0 weight, i.e., $\lambda \in P_{\mathrm{cl},+}^{0}=$ $\sum_{i \in I_{0}} \mathbf{Z}_{\geq 0} \operatorname{cl}\left(\varpi_{i}\right)$, except in the proof of Theorem 6.29. Let $V(\lambda)$ be the extremal weight module of weight $\lambda$. A priori, it is defined for $\lambda \in P^{0}$, but its $\widetilde{\mathbf{U}}$-module structure depends only on $\operatorname{cl}(\lambda)$.

\subsection{A bi-module $\widetilde{\mathbf{U}}[\lambda]$.}

Definition 6.1. For $b \in \mathcal{B}(\lambda)$, we denote by $G_{\lambda}(b)$ the corresponding element in the global basis of $V(\lambda)$. If we consider $b$ as an element of $\tilde{\mathcal{B}}$, we denote by $G(b)$ the corresponding element in $\widetilde{\mathbf{U}}$.

Denote $\#=* \circ \vee$. It is an involutive anti-automorphism of $\widetilde{\mathbf{U}}$. By [17, 4.3.2] it leaves the global crystal basis of $\widetilde{\mathbf{U}}$ invariant. (The result was proved for $*$, but the same proof works for $\vee$.) We have

Lemma $6.2(23,19.1 .1]$, 24, 3.7]). Let $x \in a_{\lambda} \widetilde{\mathbf{U}} a_{\lambda^{\prime}}$ and $\nu=\lambda-\lambda^{\prime}$. Then

$$
\psi(x)=q^{-\left(\nu, \lambda+\lambda^{\prime}\right) / 2} x^{\#} .
$$

We denote by $\leq$ the dominance partial order on the level 0 classical weights relative to the fundamental level zero weights $\operatorname{cl}\left(\varpi_{i}\right) \in P_{\mathrm{cl},+}^{0}$ defined in $\S 2.6$.

Let us give a slightly different parametrization of $\mathcal{B}(\lambda)$ from that of Sect. 1 . Let $W(\lambda), \mathcal{B}_{W}(\lambda)$ be as in the proof of Lemma 4.14. We have maps

$$
\mathcal{B}_{0}(\lambda) \stackrel{\Phi_{\lambda \mid q=0}}{\longrightarrow}\left\{\text { monomials in } z_{i, \nu}^{ \pm}\right\} \times \mathcal{B}_{W}(\lambda) \stackrel{\text { projection }}{\longrightarrow} \mathcal{B}_{W}(\lambda) .
$$

The composition is surjective since $\mathcal{B}_{W}(\lambda)$ is connected by [2, Lemmas 1.9, 1.10]. By Lemma 4.14 each fiber is identified with the set of monomials $p(z)=\prod_{i}\left(z_{i, 1} \cdots\right.$ $\left.z_{i, \lambda_{i}}\right)^{r_{i}}\left(r_{i} \in \mathbf{Z}\right)$. We choose and fix a section $\mathcal{B}_{W}(\lambda) \rightarrow \mathcal{B}_{0}(\lambda)$. (We do not require that it respect the crystal structure.) Then we have an identification (of sets, not of crystals)

$$
\mathcal{B}(\lambda) \cong \operatorname{Irr} G_{\lambda} \times \mathcal{B}_{W}(\lambda)
$$


where $G_{\lambda}=\prod_{i} G L_{\lambda_{i}}(\mathbf{C})$, and $\operatorname{Irr} G_{\lambda}$ is the set of irreducible representations of $G_{\lambda}$. We identify $\operatorname{Irr} G_{\lambda}$ with the set of Schur Laurent polynomials in $\left\{z_{i, \nu}^{ \pm}\right\}$. We denote by $s(z)$ the polynomial corresponding to $s$.

Definition 6.5. For $s \in \operatorname{Irr} G_{\lambda}$ we define $S \in G(\mathcal{B}(\lambda))$ by $\Phi_{\lambda}\left(S u_{\lambda}\right)=s(z) \tilde{u}_{\lambda}$. The existence and uniqueness are guaranteed in Theorem 4.16(ii). If $s$ is the dual of a polynomial representation and corresponds to $\mathbf{c}_{0}$, we have $S=S_{\mathbf{c}_{0}}^{-} u_{\lambda}$ by Proposition 4.10. We also regard $S$ as an element in $G\left(\mathcal{B}\left(\mathbf{U} a_{\lambda}\right)\right)$.

We will use the correspondence between $s \leftrightarrow S$ hereafter.

Definition 6.6. Let $\widetilde{\mathbf{U}}\left[{ }^{\geq} \lambda\right]$ (resp. $\widetilde{\mathbf{U}}[>\lambda]$ ) be the two-sided ideal of $\widetilde{\mathbf{U}}$ consisting of all elements $x \in \widetilde{\mathbf{U}}$ acting on $V\left(\lambda^{\prime}\right)$ by 0 for any $\lambda^{\prime} \ngtr \lambda$ (resp. $\lambda^{\prime} \ngtr \lambda$ ). Let $\tilde{\mathbf{U}}[\lambda]=\widetilde{\mathbf{U}}[\geq \lambda] / \widetilde{\mathbf{U}}[>\lambda]$. We define ${ }_{\mathcal{A}} \tilde{\mathbf{U}}[\geq \lambda],{ }_{\mathcal{A}} \tilde{\mathbf{U}}[>\lambda]$ and ${ }_{\mathcal{A}} \tilde{\mathbf{U}}[\lambda]$ in an analogous manner.

For $\xi, \xi^{\prime} \in P_{+}$let $\widetilde{\mathbf{U}}[\geq \lambda]_{\xi, \xi^{\prime}}\left(\operatorname{resp} . \widetilde{\mathbf{U}}\left[^{>} \lambda\right]_{\xi, \xi^{\prime}}\right)$ be the image of $\widetilde{\mathbf{U}}[\geq \lambda]$ (resp. $\widetilde{\mathbf{U}}[>\lambda])$ under the natural map $\widetilde{\mathbf{U}} \rightarrow V(\xi) \otimes V\left(-\xi^{\prime}\right)$ given by $x \mapsto x\left(u_{\xi} \otimes u_{-\xi^{\prime}}\right)$. Let $\widetilde{\mathbf{U}}[\lambda]_{\xi, \xi^{\prime}}=\widetilde{\mathbf{U}}[\geq \lambda]_{\xi, \xi^{\prime}} / \widetilde{\mathbf{U}}[>\lambda]_{\xi, \xi^{\prime}}$.

Lemma 6.7. (i) $\widetilde{\mathbf{U}}[\geq \lambda]$ is invariant under - .

(ii) For any $\xi, \xi^{\prime} \in P_{+}$, the $\mathbf{U}$-submodules $\widetilde{\mathbf{U}}\left[{ }^{2} \lambda\right]_{\xi, \xi^{\prime}}$ and $\widetilde{\mathbf{U}}[>\lambda]_{\xi, \xi^{\prime}}$ of $V(\xi) \otimes$ $V\left(-\xi^{\prime}\right)$ are invariant under $\widetilde{F}_{i}^{(n)}$ defined in $\oint$ 2.5.

Proof. (i) The bar involution - on $V(\lambda)$ satisfies $\overline{x u}=\bar{x} \bar{u}$ for $x \in \widetilde{\mathbf{U}}, u \in V(\lambda)$. The assertion follows.

(ii) Let $x \in \widetilde{\mathbf{U}}[\geq \lambda]$. We have

$$
\widetilde{F}_{i}^{(n)} x\left(u_{\xi} \otimes u_{-\xi^{\prime}}\right)=\sum_{k} F_{i}^{(n+k)} E_{i}^{(k)} a_{k}^{n}\left(t_{i}\right) x\left(u_{\xi} \otimes u_{-\xi^{\prime}}\right),
$$

where all but finitely many terms of the sum are 0 . Since each $F_{i}^{(n+k)} E_{i}^{(k)} a_{k}^{n}\left(t_{i}\right) x$ is contained in $\widetilde{\mathbf{U}}[\geq \lambda]$, we have the assertion.

Lemma 6.8 (cf. [24, 4.5]). (i) $\mathbf{U} a_{\lambda}, a_{\lambda} \mathbf{U} \subset \widetilde{\mathbf{U}}[\geq \lambda]$.

(ii) For $b \in \mathcal{B}\left(\mathbf{U} a_{\lambda}\right), G(b) \in \widetilde{\mathbf{U}}[>\lambda]$ if and only if $b \notin \mathcal{B}(\lambda)$.

Proof. (i) Let $x a_{\lambda} \in \mathbf{U} a_{\lambda}$. If $x a_{\lambda}$ acts on $V\left(\lambda^{\prime}\right)$ by a nonzero map, then so does $a_{\lambda}$. Since $a_{\lambda}$ is a projector to the weight space with weight $\lambda, \lambda$ is a weight of $V\left(\lambda^{\prime}\right)$. Therefore we have $\lambda \leq \lambda^{\prime}$. Similarly, we have $a_{\lambda} \mathbf{U} \subset \widetilde{\mathbf{U}}[\geq \lambda]$.

(ii) Let us first remark that $V(\lambda)_{\lambda}$ has a basis $\left\{S u_{\lambda} \mid s \in \operatorname{Irr} G_{\lambda}\right\}$. This follows from results in Sect. 因. Note that $\lambda \in P_{\mathrm{cl}}^{0}$ in this section, and the weight space is a direct sum of weight spaces $V(\lambda)_{\mu}$ with $\operatorname{cl}(\mu)=\lambda$.

Let $b \in \mathcal{B}\left(\mathbf{U} a_{\lambda}\right)$. Then $G(b)=0$ as an operator on $V(\lambda)$ if and only if $G(b) S u_{\lambda}=$ 0 for any $s \in \operatorname{Irr} G_{\lambda}$. This is equivalent to $\Phi_{\lambda}\left(G(b) S u_{\lambda}\right)=s(z) \Phi_{\lambda}\left(G(b) u_{\lambda}\right)=0$, and in particular $G(b) u_{\lambda}=0$. If $b \in \mathcal{B}(\lambda)$, then $G(b) u_{\lambda}$ is nothing but $G_{\lambda}(b)$. In particular, it is nonzero. If $b \notin \mathcal{B}(\lambda)$, then $G(b) u_{\lambda}=0$ by the definition of $V(\lambda)$.

Let $(,)_{\lambda}$ be the bilinear form on $V(\lambda)$ satisfying $(x u, v)_{\lambda}=(u, \psi(x) v)_{\lambda}$ for $u, v \in V(\lambda), x \in \widetilde{\mathbf{U}}$ and $\left(u_{\lambda}, G_{\lambda}(b)\right)_{\lambda}=1$ or 0 according to $G_{\lambda}(b)=u_{\lambda}$ or not (see Proposition 2.17).

Proposition 6.9. $\widetilde{\mathbf{U}}[\geq \lambda]$ is invariant under \#. 
Proof. Since $\widetilde{\mathbf{U}}[\geq \lambda]$ is a direct sum of its intersection with $a_{\lambda^{\prime}} \mathbf{U} a_{\lambda^{\prime \prime}}$, by $(6.3)$ it is enough to show that $x \in \widetilde{\mathbf{U}}[\geq \lambda]$ if and only if $\psi(x) \in \widetilde{\mathbf{U}}\left[{ }^{\geq} \lambda\right]$. The global crystal basis is almost orthonormal, that is $\left(G_{\lambda}(b), G_{\lambda}\left(b^{\prime}\right)\right)_{\lambda} \in \delta_{b b^{\prime}}+q_{s} \mathbf{A}_{0}$. (see Theorem 2.20). In particular, it is non-degenerate on $V(\lambda)$. Therefore, $x \in$ Ann $V(\lambda) \Longleftrightarrow\left(x G_{\lambda}(b), G_{\lambda}\left(b^{\prime}\right)\right)$ for all $b, b^{\prime} \in \mathcal{B}(\lambda) \Longleftrightarrow\left(G_{\lambda}(b), \psi(x) G_{\lambda}\left(b^{\prime}\right)\right)=$ $0 \Longleftrightarrow \psi(x) \in \operatorname{Ann} V(\lambda)$. It follows $x \in \widetilde{\mathbf{U}}[\geq \lambda]$ if and only if $\psi(x) \in \widetilde{\mathbf{U}}[\geq \lambda]$.

Remark 6.10. Since Proposition 6.9 implies $x \in \operatorname{Ann} V(\lambda)$ if and only if $x^{\#} \in$ Ann $V(\lambda)$, Lemma 6.8 implies that $b \in \mathcal{B}(\lambda)$ if and only if $G(b)^{\#} \in \widetilde{\mathbf{U}}[\geq \lambda] \backslash \widetilde{\mathbf{U}}\left[{ }^{>} \lambda\right]$ for $b \in \mathcal{B}\left(\mathbf{U} a_{\lambda}\right)$.

Note that $\widetilde{\mathbf{U}}[\lambda]=\widetilde{\mathbf{U}}\left[{ }^{\geq} \lambda\right] / \widetilde{\mathbf{U}}\left[{ }^{>} \lambda\right]$ is integrable as a $\widetilde{\mathbf{U}}$-module by Theorem 2.15 . In particular, the operators $T_{w}$ are defined.

Lemma 6.11. Let $b \in \mathcal{B}(\lambda)$. Then $G(b)^{\#} \bmod \widetilde{\mathbf{U}}[>\lambda]$ is an extremal vector in the $\widetilde{\mathbf{U}}$-module $\widetilde{\mathbf{U}}[\lambda]$.

Proof. We show that $S_{i_{1}} \cdots S_{i_{N}} G(b)^{\#} \bmod \tilde{\mathbf{U}}[>\lambda]$ is $i$-extremal for all $i \in I$ by induction on $N$.

If $N=0$, we need to show that $e_{i} G(b)^{\#}$ or $f_{i} G(b)^{\#}$ acts on $V(\lambda)$ by 0 for each $i \in I$. In other words if and only if $e_{i} G(b)^{\#}$ or $f_{i} G(b)^{\#} \in \widetilde{\mathbf{U}}[>\lambda]$. Note that $G(b)^{\#}=a_{\lambda} G(b)^{\#}$ and $a_{\lambda}$ is a projector to the weight space of weight $\lambda$. Since $V(\lambda)_{\lambda}$ has a basis $\left\{S u_{\lambda} \mid s \in \operatorname{Irr} G_{\lambda}\right\}$ consisting of extremal vectors, we are done. Moreover, we have $S_{i} G(b)^{\#} \bmod \widetilde{\mathbf{U}}[>\lambda]=a_{s_{i} \lambda} S_{i} G(b)^{\#} \bmod \widetilde{\mathbf{U}}[>\lambda]$.

We show the statement for $N$ assuming the statement for $N-1$. By the induction hypothesis and the last part of the above argument, $S_{i_{2}} \cdots S_{i_{N}} G(b)^{\#} \bmod \widetilde{\mathbf{U}}\left[{ }^{>} \lambda\right]$ is $i$-extremal for $i \in I$ and

$$
S_{i_{1}} \cdots S_{i_{N}} G(b)^{\#} \bmod \tilde{\mathbf{U}}[>\lambda]=a_{s_{i_{1}} \cdots s_{i_{N}} \lambda} S_{i_{1}} \cdots S_{i_{N}} G(b)^{\#} \bmod \tilde{\mathbf{U}}[>\lambda] .
$$

Since $V(\lambda)_{s_{i_{1}} \cdots s_{i_{N}} \lambda}$ is spanned by extremal vectors, we are done.

By Lemma 6.11, for each $b \in \mathcal{B}(\lambda)$ we have a unique $\widetilde{\mathbf{U}}$-homomorphism

$$
\Psi_{b}: V(\lambda) \rightarrow \widetilde{\mathbf{U}}[\lambda]
$$

which sends $u_{\lambda}$ to $G(b)^{\#} \bmod \widetilde{\mathbf{U}}[>\lambda]$. By definition, we have

$$
\Psi_{b}\left(G_{\lambda}\left(b_{1}\right)\right)=G\left(b_{1}\right) G(b)^{\#} \bmod \widetilde{\mathbf{U}}\left[^{>} \lambda\right] .
$$

Lemma 6.12. Let $b_{1}, b_{2} \in \mathcal{B}(\lambda)$. Then

$$
G\left(b_{1}\right)^{\#} G\left(b_{2}\right) \equiv q^{n} \sum_{s \in \operatorname{Irr} G_{\lambda}}\left(G_{\lambda}\left(b_{2}\right), G\left(b_{1}\right) S u_{\lambda}\right)_{\lambda} S a_{\lambda} \quad \bmod \tilde{\mathbf{U}}\left[{ }^{>} \lambda\right],
$$

where $n=\left(\right.$ wt $b_{1}, 2 \lambda+$ wt $\left.b_{1}\right) / 2$ and $S$ is an element corresponding to $s$ by Definition 6.9.

Proof. Since $\widetilde{\mathbf{U}}[\geq \lambda]$ is a two-sided ideal of $\widetilde{\mathbf{U}}$, both sides are in $\widetilde{\mathbf{U}}[\geq \lambda]$. Therefore it is enough to show that both sides define the same operator on $V(\lambda)$. Since we have $G\left(b_{1}\right)^{\#} G\left(b_{2}\right) u_{\lambda}=a_{\lambda} G\left(b_{1}\right)^{\#} G\left(b_{2}\right) u_{\lambda}$, it is contained in the weight space $V(\lambda)_{\lambda}$. We have a basis $\left\{S u_{\lambda}\right\}$ which is orthonormal by [30, Proposition 4.10]. Hence we have

$$
G\left(b_{1}\right)^{\#} G\left(b_{2}\right) u_{\lambda}=\sum_{s \in \operatorname{Irr} G_{\lambda}}\left(G\left(b_{1}\right)^{\#} G\left(b_{2}\right) u_{\lambda}, S u_{\lambda}\right)_{\lambda} S u_{\lambda} .
$$

Now the assertion follows from (6.3). 
Lemma 6.13. Let $S$ correspond to $s \in \operatorname{Irr} G_{\lambda}$ as in Definition 6.5. We consider it as an element in $G\left(\mathcal{B}\left(\mathbf{U} a_{\lambda}\right)\right)$. Then $S^{\#}$ corresponds to the dual representation of $s$, which will be denoted by $s^{\#}$ hereafter.

Proof. By Lemma 6.2 we have $\left(S^{\#} u_{\lambda}, S_{1} u_{\lambda}\right)=\left(u_{\lambda}, S S_{1} u_{\lambda}\right)$ for any $s_{1} \in \operatorname{Irr} G_{\lambda}$. By [30, 4.10] we have $\left(u_{\lambda}, S S_{1} u_{\lambda}\right)=\left(\Phi_{\lambda}\left(S u_{\lambda}\right), \Phi_{\lambda}\left(S S_{1} u_{\lambda}\right)\right)=\left(\tilde{u}_{\lambda}, s(z) s_{1}(z) \tilde{u}_{\lambda}\right)^{\sim}$, where $(,)^{\sim}$ is a bilinear form on $\tilde{V}(\lambda)$ defined in [loc.cit. $\S 4$ ]. By a property of $(,)^{\sim}$, we have

$$
\left(\tilde{u}_{\lambda}, s(z) s_{1}(z) \tilde{u}_{\lambda}\right)^{\sim}=\left(s\left(z^{-1}\right) \tilde{u}_{\lambda}, s_{1}(z) \tilde{u}_{\lambda}\right)^{\sim}= \begin{cases}1 & \text { if } s\left(z^{-1}\right)=s_{1}(z), \\ 0 & \text { otherwise. }\end{cases}
$$

(See an equation in the middle of [loc.cit., Proof of 4.9] and [loc.cit., Proof of 4.10]). Since $\left\{S u_{\lambda}\right\}$ is an orthonormal basis of $V(\lambda)_{\lambda}$, this means $S^{\#} u_{\lambda}$ corresponds to the Schur Laurent polynomial $s\left(z^{-1}\right)$, which corresponds to the dual representation of $s$ in turn.

Lemma 6.14. Let $(s, b) \in \operatorname{Irr} G_{\lambda} \times \mathcal{B}_{W}(\lambda)$ and let $\tilde{b}$ be the corresponding element in $B(\lambda)$ under the identification (6.4). We have

$$
G(b) S \equiv G(\tilde{b}) \bmod \tilde{\mathbf{U}}[>\lambda], \quad S^{\#} G(b)^{\#} \equiv G(\tilde{b})^{\#} \bmod \tilde{\mathbf{U}}\left[^{>} \lambda\right] .
$$

Proof. By the construction of (6.4), $\Phi_{\lambda}\left(G(\tilde{b}) u_{\lambda}\right)=s(z) \Phi_{\lambda}\left(G_{\lambda}(b)\right)=s(z) G(b) \tilde{u}_{\lambda}$. By Definition 6.5, this is equal to $G(b) \Phi_{\lambda}\left(S u_{\lambda}\right)=\Phi_{\lambda}\left(G(b) S u_{\lambda}\right)$. Therefore we have $G(b) S u_{\lambda}=G(\hat{b}) u_{\lambda}$.

Let us consider the first equation of the assertion. Since both sides of the equation are in $\mathbf{U} a_{\lambda}$, the result follows from Lemma 6.8(ii) if we prove that they define the same operators on $V(\lambda)$. Since $V(\lambda)_{\lambda}$ is spanned by $\left\{S_{1} u_{\lambda} \mid s_{1} \in \operatorname{Irr} G_{\lambda}\right\}$, it is enough to show $G(b) S S_{1} u_{\lambda}=G(\tilde{b}) S_{1} u_{\lambda}$ for every $S_{1}$. Consider the embedding $\Phi_{\lambda}: V(\lambda) \rightarrow \breve{V}(\lambda)$ as in Corollary 4.15. By Proposition 4.10 and the fact that $s_{\mathbf{c}_{0}}\left(z^{-1}\right)$ is $\widetilde{\mathbf{U}}$-linear, we have

$$
\Phi_{\lambda}\left(G(b) S S_{1} u_{\lambda}\right)=s_{1}\left(z^{-1}\right) \Phi_{\lambda}\left(G(b) S u_{\lambda}\right)=s_{1}\left(z^{-1}\right) \Phi_{\lambda}\left(G(\tilde{b}) u_{\lambda}\right)=\Phi_{\lambda}\left(G(\tilde{b}) S^{\prime} u_{\lambda}\right) .
$$

We get the assertion by the injectivity of $\Phi_{\lambda}$.

The second equation follows from the first together with Proposition 6.9.

Lemma 6.15. Let $s_{1}, s_{2} \in \operatorname{Irr} G_{\lambda}$. Let $S_{1}, S_{2} \in G\left(\mathcal{B}\left(\mathbf{U} a_{\lambda}\right)\right)$ be the corresponding elements by Definition 6.5. We have

$$
S_{1} S_{2} \equiv \sum_{s} c_{s_{1} s_{2}}^{s} S \bmod \widetilde{\mathbf{U}}\left[^{>} \lambda\right]
$$

where $s_{1} s_{2}=\sum_{s} c_{s_{1} s_{2}}^{s} s$.

Proof. Since both sides are in $\widetilde{\mathbf{U}}[\geq \lambda]$, it is enough to show that they define the same operator on $V(\lambda)$. As in the proof of Lemma 6.14, it follows from $S_{1} S_{2} u_{\lambda}=$ $\sum_{s} c_{s_{1} s_{2}}^{s} S u_{\lambda}$. But this follows directly from Definition 6.5.

Definition 6.16. For $b, b^{\prime} \in \mathcal{B}_{W}(\lambda), s \in \operatorname{Irr} G_{\lambda}$ we set

$$
G_{\lambda}\left(b, s, b^{\prime}\right)=G(b) S G\left(b^{\prime}\right)^{\#} \bmod \tilde{\mathbf{U}}[>\lambda]=\Psi_{b^{\prime}}\left(G(b) S u_{\lambda}\right) \in \widetilde{\mathbf{U}}[\lambda] .
$$

The following is a direct consequence of Lemma 6.12. 
Lemma 6.17. We have

$$
\begin{aligned}
& G_{\lambda}\left(b_{1}, s_{1}, b_{1}^{\prime}\right) G_{\lambda}\left(b_{2}, s_{2}, b_{2}^{\prime}\right) \\
= & q^{n} \sum_{s^{\prime \prime} \in \operatorname{Irr} G_{\lambda}}\left(G\left(b_{2}\right) S_{2} u_{\lambda}, G\left(b_{1}^{\prime}\right) S^{\prime \prime} u_{\lambda}\right)_{\lambda} G\left(b_{1}\right) S_{1} S^{\prime \prime} G\left(b_{2}^{\prime}\right)^{\#} \bmod \widetilde{\mathbf{U}}\left[{ }^{>} \lambda\right],
\end{aligned}
$$

where $n=\left(\right.$ wt $\left.b_{1}^{\prime}, 2 \lambda+\mathrm{wt} b_{1}^{\prime}\right) / 2$.

Lemma 6.18. $\left\{G_{\lambda}\left(b, s, b^{\prime}\right) \mid b, b^{\prime} \in \mathcal{B}_{W}(\lambda), s \in \operatorname{Irr} G_{\lambda}\right\}$ is linearly independent.

Proof. Suppose that $\sum_{b, b^{\prime}, s} a_{b, b^{\prime}, s} G(b) S G\left(b^{\prime}\right)^{\#}$ acts on $V(\lambda)$ by 0 . Then for any $b_{1} \in \mathcal{B}(\lambda)$

$$
\begin{aligned}
0 & =\sum_{b, b^{\prime}, s} a_{b, b^{\prime}, s} G(b) S G\left(b^{\prime}\right)^{\#} G_{\lambda}\left(b_{1}\right) \\
& =\sum_{b, b^{\prime}, s} a_{b, b^{\prime}, s} G(b)\left(G\left(b^{\prime}\right) S^{\#}\right)^{\#} G_{\lambda}\left(b_{1}\right) \\
& =\sum_{b, b^{\prime}, s, s^{\prime}} a_{b, b^{\prime}, s} q^{n\left(b^{\prime}\right)}\left(G_{\lambda}\left(b_{1}\right), G\left(b^{\prime}\right) S^{\#} S^{\prime} u_{\lambda}\right)_{\lambda} G(b) S^{\prime} u_{\lambda},
\end{aligned}
$$

where $n\left(b^{\prime}\right)=\left(\mathrm{wt} b^{\prime}, 2 \lambda+\mathrm{wt} b^{\prime}\right) / 2$. Here we have used Lemma 6.12 in the third equality. Since $\left\{G(b) S^{\prime} u_{\lambda} \mid b \in \mathcal{B}_{W}(\lambda), s^{\prime} \in \operatorname{Irr} G_{\lambda}\right\}$ is linearly independent, we have

$$
\sum_{b^{\prime}, s} a_{b, b^{\prime}, s} q^{n\left(b^{\prime}\right)}\left(G_{\lambda}\left(b_{1}\right), G\left(b^{\prime}\right) S^{\#} S^{\prime} u_{\lambda}\right)_{\lambda}=0
$$

for any $b, b_{1}, s^{\prime}$. This equality for $s^{\prime}=1$, together with the nondegeneracy of $(,)_{\lambda}$ and linearly independence of $\left\{G\left(b^{\prime}\right) S^{\#} u_{\lambda}\right\}$ imply $a_{b, b^{\prime}, s}=0$.

Definition 6.19. For $\xi, \xi^{\prime} \in P_{+}$, let $\tilde{\mathcal{L}}[\geq \lambda]_{\xi, \xi^{\prime}}=\left(\mathcal{L}(\xi) \otimes \mathcal{L}\left(-\xi^{\prime}\right)\right) \cap \widetilde{\mathbf{U}}[\geq \lambda]_{\xi, \xi^{\prime}}$ and $\tilde{\mathcal{L}}\left[^{>} \lambda\right]_{\xi, \xi^{\prime}}=\left(\mathcal{L}(\xi) \otimes \mathcal{L}\left(-\xi^{\prime}\right)\right) \cap \tilde{\mathbf{U}}\left[{ }^{>} \lambda\right]_{\xi, \xi^{\prime}}$. Let $\left.\tilde{\mathcal{L}}[\lambda]_{\xi, \xi^{\prime}}=\tilde{\mathcal{L}}\left[{ }^{\geq} \lambda\right]_{\xi, \xi^{\prime}} / \tilde{\mathcal{L}} \Gamma^{>} \lambda\right]_{\xi, \xi^{\prime}}$. Let $\tilde{\mathcal{L}}[\lambda]$ be the $\mathbf{A}_{0}$-submodule of $\widetilde{\mathbf{U}}[\lambda]$ consisting of elements whose images under $\widetilde{\mathbf{U}}[\lambda] \rightarrow \widetilde{\mathbf{U}}[\lambda]_{\xi, \xi^{\prime}}$ are in $\tilde{\mathcal{L}}[\lambda]_{\xi, \xi^{\prime}}$ for any $\xi, \xi^{\prime} \in P_{+}$. Let $\tilde{\mathcal{L}}[\geq \lambda]$ be the $\mathbf{A}_{0}$-submodule of $\widetilde{\mathbf{U}}[\geq \lambda]$ consisting of elements whose images under $\widetilde{\mathbf{U}}[\geq \lambda] \rightarrow \widetilde{\mathbf{U}}[\geq \lambda]_{\xi, \xi^{\prime}}$ are in $\tilde{\mathcal{L}}[\geq \lambda]_{\xi, \xi^{\prime}}$ for any $\xi, \xi^{\prime} \in P_{+}$. By the definition of $\tilde{\mathcal{L}}$ (see [23, Part IV]), this is equal to $\tilde{\mathcal{L}} \cap \widetilde{\mathbf{U}}[\geq \lambda]$. We define $\tilde{\mathcal{L}}[>\lambda]$ in the similar way.

Lemma 6.20. $\Psi_{b^{\prime}}(\mathcal{L}(\lambda)) \subset \tilde{\mathcal{L}}[\lambda]$ for $b^{\prime} \in \mathcal{B}_{W}(\lambda)$.

Proof. The homomorphism $\Psi_{b^{\prime}}: V(\lambda) \rightarrow \widetilde{\mathbf{U}}[\lambda]$ intertwines the operators $\tilde{e}_{i}, \tilde{f}_{i}$. Since $\tilde{\mathcal{L}}[\lambda]$ is invariant under $\tilde{e}_{i}, \tilde{f}_{i}$ (see Lemma 6.7(ii)), it is enough to show that $\Psi_{b^{\prime}}\left(S u_{\lambda}\right) \in \tilde{\mathcal{L}}[\lambda]$. This follows from Lemma 6.14 as $\Psi_{b^{\prime}}\left(S u_{\lambda}\right)=S G\left(b^{\prime}\right)^{\#} \bmod$ $\left.\widetilde{\mathbf{U}} \Gamma^{>} \lambda\right]$.

Most importantly, we have $G_{\lambda}\left(b, s, b^{\prime}\right) \in \tilde{\mathcal{L}}[\lambda]$. Also more generally, Lemma 6.20 holds for $b^{\prime} \in \mathcal{B}(\lambda)$ thanks to Lemma 6.15.

Definition 6.21. Let $\tilde{\mathcal{B}}[\lambda]$ be the subset of $\tilde{\mathcal{B}}$ consisting of elements which are connected to $\mathcal{B}(\lambda)^{\#}$ in the crystal graph. It has an induced bi-crystal structure from $\tilde{\mathcal{B}}$. By Theorem $4.16 \tilde{\mathcal{B}}[\lambda]=\tilde{\mathcal{B}}[\mu]$ if $\mu \in \hat{W} \lambda, \tilde{\mathcal{B}}[\lambda] \cap \tilde{\mathcal{B}}[\mu]=\emptyset$ otherwise. In particular, $\tilde{\mathcal{B}}[\lambda]\left(\lambda \in P_{\mathrm{cl},+}^{0}\right)$ are mutually disjoint. 
Remark 6.22. We will see that this definition coincides with a more obvious definition of $\tilde{\mathcal{B}}[\lambda]$, i.e., the set of all elements $b \in \tilde{\mathcal{B}}$ such that $G(b) \in \widetilde{\mathbf{U}}[\geq \lambda]$ and such that $G(b)$ acts on $V(\lambda)$ non-trivially. We will show, in fact, that $\widetilde{\mathbf{U}}[\lambda]$ has a bi-crystal basis given by $\tilde{\mathcal{B}}[\lambda]$.

As a first step we have

Proposition 6.23. There exists an isomorphism of bi-crystals

$$
\tilde{\mathcal{B}}[\lambda] \cong \mathcal{B}_{W}(\lambda) \times \operatorname{Irr} G_{\lambda} \times \mathcal{B}_{W}(\lambda)
$$

where $\tilde{e}_{i}, \tilde{f}_{i}, \tilde{e}_{i}^{\#}, \tilde{f}_{i}^{\#}$ of the right hand side are defined by

$$
\begin{gathered}
\tilde{e}_{i}\left(b, s, b^{\prime}\right)=\left(\tilde{e}_{i}(b, s), b^{\prime}\right), \quad \tilde{f}_{i}\left(b, s, b^{\prime}\right)=\left(\tilde{f}_{i}(b, s), b^{\prime}\right), \\
\tilde{e}_{i}^{\#}\left(b, s, b^{\prime}\right)=\left(b,(\operatorname{id} \times \#) \circ \tilde{e}_{i} \circ(\operatorname{id} \times \#)\left(b^{\prime}, s\right)\right), \\
\tilde{f}_{i}^{\#}\left(b, s, b^{\prime}\right)=\left(b,(\operatorname{id} \times \#) \circ \tilde{f}_{i} \circ(\operatorname{id} \times \#)\left(b^{\prime}, s\right)\right),
\end{gathered}
$$

where we use the identification $\mathcal{B}(\lambda) \cong B_{W}(\lambda) \times \operatorname{Irr} G_{\lambda}$ of (6.4). Here $s^{\#}$ denotes the dual representation of $s$.

Proof. There is a set-theoretical bijection between the right hand side and the subset

$$
\left\{G_{\lambda}\left(b, s, b^{\prime}\right) \bmod q_{s} \tilde{\mathcal{L}}[\lambda] \mid b, b^{\prime} \in \mathcal{B}_{W}(\lambda), s \in \operatorname{Irr} G_{\lambda}\right\} \subset \tilde{\mathcal{L}}[\lambda] / q_{s} \tilde{\mathcal{L}}[\lambda] .
$$

The $\widetilde{\mathbf{U}}$-module structure on $\widetilde{\mathbf{U}}[\lambda]$ defines operators $\tilde{e}_{i}, \tilde{f}_{i}$ on $\tilde{\mathcal{L}}[\lambda] / q_{s} \tilde{\mathcal{L}}[\lambda]$. If we write

$$
G_{\lambda}\left(b, s, b^{\prime}\right)=G(\tilde{b}) G\left(b^{\prime}\right)^{\#} \bmod \tilde{\mathbf{U}}\left[{ }^{>} \lambda\right]=\Psi_{b^{\prime}}\left(G_{\lambda}(\tilde{b})\right),
$$

with $\tilde{b}=(b, s) \in \mathcal{B}(\lambda)$ defined by (6.4), then

$$
X_{i} G_{\lambda}\left(b, s, b^{\prime}\right)=\Psi_{b^{\prime}}\left(X_{i} G_{\lambda}(\tilde{b})\right) \equiv \Psi_{b^{\prime}}\left(G_{\lambda}\left(X_{i} \tilde{b}\right)\right) \bmod q_{s} \tilde{\mathcal{L}}[\lambda],
$$

for $X_{i}=\tilde{e}_{i}$ or $\tilde{f}_{i}$. Here we have used Lemma 6.20 for the second equality. This shows that the bijection respects the crystal structure. The identification of \#crystal structure follows from the above discussion and

$$
G_{\lambda}\left(b, s, b^{\prime}\right)^{\#}=G\left(b^{\prime}\right) S^{\#} G(b)^{\#} \bmod \tilde{\mathbf{U}}[>\lambda],
$$

together with Lemma 6.13. We identify $\mathcal{B}_{W}(\lambda) \times \operatorname{Irr} G_{\lambda} \times \mathcal{B}_{W}(\lambda)$ with 6.24 hereafter.

Consider the natural Q-linear map

$$
\pi: \tilde{\mathcal{L}}[\geq \lambda] / q_{s} \tilde{\mathcal{L}}[\geq \lambda] \rightarrow \tilde{\mathcal{L}}[\lambda] / q_{s} \tilde{\mathcal{L}}[\lambda]
$$

induced by the projection $\widetilde{\mathbf{U}}[\geq \lambda] \rightarrow \widetilde{\mathbf{U}}[\lambda]$. Recall that the Kashiwara operators $\tilde{e}_{i}, \tilde{f}_{i}$ on $\tilde{\mathcal{L}} / q_{s} \tilde{\mathcal{L}}$ are defined so that they are compatible with projections $\tilde{\mathcal{L}} / q_{s} \tilde{\mathcal{L}} \rightarrow$ $\mathcal{L}(\xi) \otimes \mathcal{L}\left(-\xi^{\prime}\right) / q_{s}\left(\mathcal{L}(\xi) \otimes \mathcal{L}\left(-\xi^{\prime}\right)\right)$ induced from $\widetilde{\mathbf{U}} \rightarrow V(\xi) \otimes V\left(-\xi^{\prime}\right)$ for all $\xi, \xi^{\prime} \in P_{+}$ (see 17, Theorem 2.1.2] and [23, Part IV]). Since $\tilde{\mathcal{L}}\left[{ }^{\geq} \lambda\right]_{\xi, \xi^{\prime}}$ is invariant under $\widetilde{F}_{i}^{(n)}$ by Lemma 6.7(ii), the subspace $\tilde{\mathcal{L}}[\geq \lambda] / q_{s} \tilde{\mathcal{L}}[\geq \lambda] \subset \tilde{\mathcal{L}} / q_{s} \tilde{\mathcal{L}}$ is invariant under $\tilde{e}_{i}, \tilde{f}_{i}$. The map $\pi$ intertwines $\tilde{e}_{i}, \tilde{f}_{i}$ as $\tilde{\mathcal{L}}[\geq \lambda]_{\xi, \xi^{\prime}} / q_{s} \tilde{\mathcal{L}}[\geq \lambda]_{\xi, \xi^{\prime}} \rightarrow \tilde{\mathcal{L}}[\lambda]_{\xi, \xi^{\prime}} / q_{s} \tilde{\mathcal{L}}[\lambda]_{\xi, \xi^{\prime}}$ does so. It also intertwines \#, and hence $\tilde{e}_{i}^{\#}, \tilde{f}_{i}^{\#}$.

Since $G(b)^{\#} \in \widetilde{\mathbf{U}}\left[{ }^{\geq} \lambda\right]$ for $b \in \mathcal{B}(\lambda), \tilde{\mathcal{B}}[\lambda]$ is contained in $\tilde{\mathcal{L}}\left[{ }^{\geq} \lambda\right] / q_{s} \tilde{\mathcal{L}}[\geq \lambda]$ as it is invariant under $\tilde{e}_{i}, \tilde{f}_{i}$. We have $\pi\left(b^{\#}\right)=\left(u_{\lambda}, s, b^{\prime}\right)$, where $b=\left(s^{\#}, b^{\prime}\right)$ under (6.4). Therefore we have

$$
\pi(\tilde{\mathcal{B}}[\lambda]) \subset \mathcal{B}_{W}(\lambda) \times \operatorname{Irr} G_{\lambda} \times \mathcal{B}_{W}(\lambda) \sqcup\{0\} .
$$


Consider $\operatorname{Ker} \pi \cap \tilde{\mathcal{B}}[\lambda]$. It is invariant under $\tilde{e}_{i}, \tilde{f}_{i}$, so every connected component contains an extremal vector, and in particular an element in $\mathcal{B}(\lambda)^{\#}$. But $\mathcal{B}(\lambda)^{\#}$ is mapped bijectively to $\left\{u_{\lambda}\right\} \times \operatorname{Irr} G_{\lambda} \times \mathcal{B}_{W}(\lambda)$ as we mentioned already. So $\operatorname{Ker} \pi \cap$ $\tilde{\mathcal{B}}[\lambda]$ is the empty set. Thus we have a map

$$
\left.\pi\right|_{\tilde{\mathcal{B}}[\lambda]}: \tilde{\mathcal{B}}[\lambda] \rightarrow \mathcal{B}_{W}(\lambda) \times \operatorname{Irr} G_{\lambda} \times \mathcal{B}_{W}(\lambda) .
$$

It is enough to show that this map is bijective since it is clear that bi-crystal operators are intertwined.

Note that any element of $\mathcal{B}_{W}(\lambda) \times \operatorname{Irr} G_{\lambda} \times \mathcal{B}_{W}(\lambda)$ can be connected to a point in $\left\{u_{\lambda}\right\} \times \operatorname{Irr} G_{\lambda} \times \mathcal{B}_{W}(\lambda)$ in the crystal graph, since the assertion is so for $\mathcal{B}(\lambda)$ (Theorem 4.16). Since $\left\{u_{\lambda}\right\} \times \operatorname{Irr} G_{\lambda} \times \mathcal{B}_{W}(\lambda)$ is equal to $\pi\left(\mathcal{B}(\lambda)^{\#}\right)$, the map $\left.\pi\right|_{\tilde{\mathcal{B}}[\lambda]}$ is surjective.

Suppose that $b, b^{\prime} \in \tilde{\mathcal{B}}[\lambda]$ satisfy $\pi(b)=\pi\left(b^{\prime}\right)$. We may assume that $b \in \mathcal{B}(\lambda)^{\#}$. The condition $\pi(b)=\pi\left(b^{\prime}\right)$ and $\operatorname{Ker} \pi \cap \tilde{\mathcal{B}}[\lambda]=\emptyset$ imply $b^{\prime}$ is an extremal vector with the weight wt $b^{\prime}=$ wt $b$. This means $b^{\prime} \in \mathcal{B}(\lambda)^{\#}$. Since $\pi$ is bijective on $\mathcal{B}(\lambda)^{\#}$, we have $b=b^{\prime}$.

Hereafter, we identify $\tilde{\mathcal{B}}[\lambda]$ with $\mathcal{B}_{W}(\lambda) \times \operatorname{Irr} G_{\lambda} \times \mathcal{B}_{W}(\lambda)$ by Proposition 6.23.

Lemma 6.25. $\tilde{\mathcal{B}}=\bigsqcup_{\lambda \in P_{\mathrm{cl}}^{0,+}} \tilde{\mathcal{B}}[\lambda]$.

Proof. Each connected component of $\tilde{\mathcal{B}}$ contains an extremal vector [17, 9.3.4]. By definition, the set of extremal vectors is equal to $\bigsqcup_{\lambda \in P_{\mathrm{cc}}^{0}} \mathcal{B}(\lambda)^{*}=\bigsqcup_{\lambda \in P_{\mathrm{cl}}^{0}} \mathcal{B}(\lambda)^{\#}$. Furthermore, we have an isomorphism of crystals $S_{w}^{*}: \mathcal{B}(\lambda) \cong \mathcal{B}(w \lambda)$ for $w \in \hat{W}$. Therefore each component contains a vector in $\mathcal{B}(\lambda)^{\#}$ with $\lambda \in P_{\mathrm{cl},+}^{0}$. Therefore $\tilde{\mathcal{B}}=\bigcup_{\lambda \in P_{\mathrm{cl}}^{0,+}} \tilde{\mathcal{B}}[\lambda]$. By the remark in Definition 6.21, this is a disjoint union.

For $\xi, \xi^{\prime} \in P_{+}$, the kernel of the surjective homomorphism $\widetilde{\mathbf{U}} a_{\xi-\xi^{\prime}} \rightarrow V(\xi) \otimes$ $V\left(-\xi^{\prime}\right)$ is the left ideal generated by $f_{i}^{\left\langle\xi, h_{i}\right\rangle+1} a_{\xi-\xi^{\prime}}$ and $e_{i}^{\left\langle\xi^{\prime}, h_{i}\right\rangle+1} a_{\xi-\xi^{\prime}}$. Let $P\left(\xi, \xi^{\prime}\right)$ be the set of $\mu \in P_{\mathrm{cl},+}^{0}$ such that all $f_{i}^{\left\langle\xi, h_{i}\right\rangle+1} a_{\xi-\xi^{\prime}}$ and $e_{i}^{\left\langle\xi^{\prime}, h_{i}\right\rangle+1} a_{\xi-\xi^{\prime}}$ act by 0 on $V\left(\mu^{\prime}\right)$ for any $\mu^{\prime} \in P_{\mathrm{cl},+}^{0}$ satisfying $\mu^{\prime} \leq \mu$. Therefore a homomorphism $V(\xi) \otimes$ $V\left(-\xi^{\prime}\right) \rightarrow$ End $V(\mu)$ is well-defined if $\mu \in P\left(\xi, \xi^{\prime}\right)$. Moreover, any $\mu \in P_{\mathrm{cl},+}^{0}$ is contained in $P\left(\xi, \xi^{\prime}\right)$ for sufficiently large $\xi, \xi^{\prime}$.

Let $\tilde{\mathcal{B}}[\mu]_{\xi, \xi^{\prime}}=\tilde{\mathcal{B}}[\mu] \cap(\mathcal{B}(\xi) \otimes \mathcal{B}(-\xi))$. Then we have $\mathcal{B}(\xi) \otimes \mathcal{B}\left(-\xi^{\prime}\right)=\bigsqcup_{\mu} \tilde{\mathcal{B}}[\mu]_{\xi, \xi^{\prime}}$ by Lemma 6.25 .

Let $\left(b, s, b^{\prime}\right) \in \tilde{\mathcal{B}}[\mu]_{\xi, \xi^{\prime}}$. We have $G_{\mu}\left(b, s, b^{\prime}\right)\left(u_{\xi} \otimes u_{-\xi^{\prime}}\right) \in \tilde{\mathcal{L}}[\mu]_{\xi, \xi^{\prime}}$ by Lemma 6.20. We choose lifts $\tilde{G}_{\mu}\left(b, s, b^{\prime}\right) \in \tilde{\mathcal{L}}[\geq \mu]_{\xi, \xi^{\prime}}$. (Note that we do not have $G(b) S G\left(b^{\prime}\right)^{\#} \in \mathcal{L}$ in general.) Then

$$
\bigsqcup_{\mu \in P_{\mathrm{cl}}^{0,+}}\left\{\tilde{G}_{\mu}\left(b, s, b^{\prime}\right) \mid\left(b, s, b^{\prime}\right) \in \tilde{\mathcal{B}}[\mu]_{\xi, \xi^{\prime}}\right\}
$$

is a $\mathbf{A}_{0}$-basis of $\mathcal{L}(\xi) \otimes \mathcal{L}\left(\xi^{\prime}\right)$, as it induces a $\mathbf{Q}$-basis of $\mathcal{L}(\xi) \otimes \mathcal{L}\left(\xi^{\prime}\right) / q_{s}\left(\mathcal{L}(\xi) \otimes \mathcal{L}\left(\xi^{\prime}\right)\right)$. (The transition matrix between this basis and $\mathcal{B}(\xi) \otimes \mathcal{B}\left(-\xi^{\prime}\right)$ is upper triangular with 1's on the diagonal with respect to the block decomposition induced by $\mu$ and the order $\leq$.) 
Lemma 6.26. Let $\tilde{G}_{\mu}\left(b, s, b^{\prime}\right) \in \tilde{\mathcal{L}}[\mu]_{\xi, \xi^{\prime}}$ as above. If

$$
x=\sum_{\mu \in P_{\mathrm{cl}}^{0,+}} \sum_{\left(b, s, b^{\prime}\right) \in \tilde{\mathcal{B}}[\mu]_{\xi, \xi^{\prime}}} a_{b, s, b^{\prime}}^{\mu} \tilde{G}_{\mu}\left(b, s, b^{\prime}\right) \in \tilde{\mathbf{U}}[\geq \lambda]_{\xi, \xi^{\prime}},
$$

then $a_{b, s, b^{\prime}}^{\mu}=0$ if $\mu \nsupseteq \lambda$ and $\mu \in P\left(\xi, \xi^{\prime}\right)$.

Proof. We take any minimal element $\mu_{0}$ with respect to $\leq$ among $\mu$ 's with $a_{b, s, b^{\prime}}^{\mu} \neq 0$ for some $\left(b, s, b^{\prime}\right) \in \tilde{\mathcal{B}}[\mu]$. Assume $\mu_{0} \nsucceq \lambda$ and $\mu_{0} \in P\left(\xi, \xi^{\prime}\right)$. Then $V(\xi) \otimes V\left(-\xi^{\prime}\right) \rightarrow$ $\operatorname{End} V\left(\mu_{0}\right)$ is well-defined, and the image of $x$ is 0 by the assumption. On the other hand, any $\mu$ with $a_{b, s, b^{\prime}}^{\mu} \neq 0$ for some $\left(b, s, b^{\prime}\right) \in \tilde{\mathcal{B}}[\mu]$ satisfies $\mu \nless \mu_{0}$ by the minimality condition. Therefore among terms in $x$, only those summands with $\mu=\mu_{0}$ act nontrivially on $V\left(\mu_{0}\right)$. Therefore, as operators on $V\left(\mu_{0}\right)$, we have

$$
\left.x\right|_{V\left(\mu_{0}\right)}=\left.\sum_{\left(b, s, b^{\prime}\right) \in \tilde{\mathcal{B}}\left[\mu_{0}\right]_{\xi, \xi^{\prime}}} a_{b, s, b^{\prime}}^{\mu_{0}} \tilde{G}_{\mu_{0}}\left(b, s, b^{\prime}\right)\right|_{V\left(\mu_{0}\right)}=0 .
$$

However the operator $\left.\tilde{G}_{\mu_{0}}\left(b, s, b^{\prime}\right)\right|_{V\left(\mu_{0}\right)}$ does not depend on the choice of the lift $\tilde{G}_{\mu_{0}}\left(b, s, b^{\prime}\right)$ of $G_{\mu_{0}}\left(b, s, b^{\prime}\right)$, and we have $a_{b, s, b^{\prime}}^{\mu_{0}}=0$ for $\left(b, s, b^{\prime}\right) \in \tilde{\mathcal{B}}\left[\mu_{0}\right]_{\xi, \xi^{\prime}}$, as $G_{\mu_{0}}\left(b, s, b^{\prime}\right)$ are linearly independent (Lemma 6.18). This is a contradiction. Thus we have $\mu_{0} \geq \lambda$ or $\mu_{0} \notin P\left(\xi, \xi^{\prime}\right)$. Since $\mu_{0}$ is any minimal element, this completes the proof.

Proposition 6.27. Let $\beta=\left(b, s, b^{\prime}\right) \in \tilde{\mathcal{B}}[\lambda]$. Considering $\beta$ as an element of $\tilde{\mathcal{B}}$, let $G(\beta)$ be the corresponding element of the global crystal basis of $\widetilde{\mathbf{U}}$. Then we have

$$
G(\beta) \in \widetilde{\mathbf{U}}[\geq \lambda], \quad G(\beta) \bmod \widetilde{\mathbf{U}}[>\lambda]=G_{\lambda}\left(b, s, b^{\prime}\right) .
$$

Proof. We will show the assertion by showing

(a) $G(\beta)\left(u_{\xi} \otimes u_{-\xi^{\prime}}\right)$ acts by 0 on $V(\mu)$ if $\mu \nsupseteq \lambda$ and $\mu \in P\left(\xi, \xi^{\prime}\right)$.

(b) $G(\beta)\left(u_{\xi} \otimes u_{-\xi^{\prime}}\right)$ and $G_{\lambda}\left(b, s, b^{\prime}\right)\left(u_{\xi} \otimes u_{-\xi^{\prime}}\right)$ define the same operator on $V(\lambda)$ if $\lambda \in P\left(\xi, \xi^{\prime}\right)$,

for any $\xi, \xi^{\prime} \in P_{+}$. We fix $\xi, \xi^{\prime}$ hereafter.

Let $\left(b_{1}, s_{1}, b_{1}^{\prime}\right) \in \tilde{\mathcal{B}}[\mu]_{\xi, \xi^{\prime}}$. We choose and fix a lift $\tilde{G}_{\mu}\left(b_{1}, s_{1}, b_{1}^{\prime}\right) \in \tilde{\mathcal{L}}\left[{ }^{\geq} \mu\right]_{\xi, \xi^{\prime}}$ of $G_{\mu}\left(b_{1}, s_{1}, b_{1}^{\prime}\right)\left(u_{\xi} \otimes u_{-\xi^{\prime}}\right)$ as in Lemma 6.26. We write

$$
G(\beta)\left(u_{\xi} \otimes u_{-\xi^{\prime}}\right)=\sum_{\mu \in P_{\mathrm{cl},+}^{0}} \sum_{\left(b_{1}, s_{1}, b_{1}^{\prime}\right) \in \tilde{\mathcal{B}}[\mu]_{\xi, \xi^{\prime}}} a_{b_{1}, s_{1}, b_{1}^{\prime}}^{\mu} \tilde{G}_{\mu}\left(b_{1}, s_{1}, b_{1}^{\prime}\right)
$$

for some $a_{b_{1}, s_{1}, b_{1}^{\prime}}^{\mu} \in \mathbf{A}_{0}$.

Claim. (i) If $\mu \nsupseteq \lambda$ and $\mu \in P\left(\xi, \xi^{\prime}\right)$, then $a_{b_{1}, s_{1}, b_{1}^{\prime}}^{\mu} \in q_{s} \mathbf{A}_{0}$ for $\left(b_{1}, s_{1}, b_{1}^{\prime}\right) \in \tilde{\mathcal{B}}[\mu]_{\xi, \xi^{\prime}}$.

(ii) If $\lambda \in P\left(\xi, \xi^{\prime}\right)$, then $a_{b_{1}, s_{1}, b_{1}^{\prime}}^{\lambda} \in \delta_{\left(b, s, b^{\prime}\right),\left(b_{1}, s_{1}, b_{1}^{\prime}\right)}+q_{s} \mathbf{A}_{0}$ for $\left(b_{1}, s_{1}, b_{1}^{\prime}\right) \in$ $\tilde{\mathcal{B}}[\lambda]_{\xi, \xi^{\prime}} \cdot$

We can replace $G(\beta)\left(u_{\xi} \otimes u_{-\xi^{\prime}}\right)$ by an element which is equal to it modulo $q_{s}\left(\mathcal{L}(\xi) \otimes \mathcal{L}\left(-\xi^{\prime}\right)\right)$ in showing (i) and (ii). Let us write

$$
(b, s)=X_{1} \cdots X_{N}\left(u_{\lambda}, s_{0}\right)
$$

for some $X_{i}=\tilde{e}_{j}$ or $\tilde{f}_{j}$. Then $G(\beta)\left(u_{\xi} \otimes u_{-\xi^{\prime}}\right)$ is equal to $X_{1} \cdots X_{N} S_{0} G\left(b^{\prime}\right)^{\#}\left(u_{\xi} \otimes\right.$ $u_{-\xi^{\prime}}$ ) modulo $q_{s}\left(\mathcal{L}(\xi) \otimes \mathcal{L}\left(-\xi^{\prime}\right)\right)$. We show the assertion for this element. By Lemma 6.7, it is contained in $\widetilde{\mathbf{U}}[\geq \lambda]_{\xi, \xi^{\prime}}$. By Lemma 6.26 we have (i). 
To show (ii), suppose $\lambda \in P\left(\xi, \xi^{\prime}\right)$ and $V(\xi) \otimes V\left(\xi^{\prime}\right) \rightarrow$ End $V(\lambda)$ is well-defined. If $\mu \notin P\left(\xi, \xi^{\prime}\right)$, then $\lambda \ngtr \mu$ by the assumption. We combine this with the above discussion to have $a_{b_{1}, s_{1}, b_{1}^{\prime}}^{\mu}=0$ unless $\lambda \ngtr \mu$. Therefore among terms in (6.28), only those summands with $\mu=\lambda$ act nontrivially on $V(\lambda)$. Thus we have

$$
\begin{aligned}
X_{1} \cdots X_{N} S_{0} G\left(b^{\prime}\right)^{\#}\left(u_{\xi}\right. & \left.\otimes u_{-\xi^{\prime}}\right) \\
& \equiv \sum_{\left(b_{1}, s_{1}, b_{1}^{\prime}\right) \in \tilde{\mathcal{B}}[\lambda]_{\xi, \xi^{\prime}}} a_{b_{1}, s_{1}, b_{1}^{\prime}}^{\lambda} \tilde{G}_{\lambda}\left(b_{1}, s_{1}, b_{1}^{\prime}\right) \quad \bmod \tilde{\mathbf{U}}[>\lambda]_{\xi, \xi^{\prime}} .
\end{aligned}
$$

On the other hand, we have

$$
X_{1} \cdots X_{N} S_{0} G\left(b^{\prime}\right)^{\#} \bmod \tilde{\mathbf{U}}\left[{ }^{>} \lambda\right]=\Psi_{b^{\prime}}\left(X_{1} \cdots X_{N} S_{0} u_{\lambda}\right) .
$$

Since $\Psi_{b^{\prime}}(\tilde{\mathcal{L}}(\lambda)) \subset \tilde{\mathcal{L}}[\lambda]$ by Lemma 6.20 , this is equal to $G_{\lambda}\left(b, s, b^{\prime}\right)$ modulo $q_{s} \tilde{\mathcal{L}}[\lambda]$. This completes the proof of the claim.

We take any minimal element $\mu_{0}$ with respect to $\leq$ among $\mu$ 's with $a_{b_{1}, s_{1}, b_{1}^{\prime}}^{\mu} \neq 0$ for some $\left(b_{1}, s_{1}, b_{1}^{\prime}\right) \in \tilde{\mathcal{B}}[\mu]$. We obtain a contradiction under the assumption $\mu_{0} \ngtr \lambda$ and $\mu_{0} \in P\left(\xi, \xi^{\prime}\right)$. As in the proof of Lemma 6.26, only summands with $\mu=\mu_{0}$ act nontrivially on $V\left(\mu_{0}\right)$. Hence, for $b^{\prime \prime} \in \mathcal{B}\left(\mu_{0}\right)$ we have

$$
\begin{aligned}
G(\beta) G_{\mu_{0}}\left(b^{\prime \prime}\right)= & \sum_{\left(b_{1}, s_{1}, b_{1}^{\prime}\right) \in \tilde{\mathcal{B}}\left[\mu_{0}\right]} a_{b_{1}, s_{1}, b_{1}^{\prime}}^{\mu_{0}} \tilde{G}_{\mu_{0}}\left(b_{1}, s_{1}, b_{1}^{\prime}\right) G_{\mu_{0}}\left(b^{\prime \prime}\right) \\
= & \sum_{\left(b_{1}, s_{1}, b_{1}^{\prime}\right) \in \tilde{\mathcal{B}}\left[\mu_{0}\right]} a_{b_{1}, s_{1}, b_{1}^{\prime}}^{\mu_{0}} G\left(b_{1}\right) S_{1} G\left(b_{1}^{\prime}\right)^{\#} G_{\mu_{0}}\left(b^{\prime \prime}\right) .
\end{aligned}
$$

From $\overline{G(\beta) G_{\mu_{0}}\left(b^{\prime \prime}\right)}=G(\beta) G_{\mu_{0}}\left(b^{\prime \prime}\right)$, we have

$$
0=\sum_{\left(b_{1}, s_{1}, b_{1}^{\prime}\right) \in \tilde{\mathcal{B}}\left[\mu_{0}\right]}\left(a_{b_{1}, s_{1}, b_{1}^{\prime}}^{\mu_{0}}-\overline{a_{b_{1}, s_{1}, b_{1}^{\prime}}^{\mu_{0}}}\right) G\left(b_{1}\right) S_{1} G\left(b_{1}^{\prime}\right)^{\#} G_{\mu_{0}}\left(b^{\prime \prime}\right) .
$$

Arguing as in the proof of Lemma 6.18, we get

$$
a_{b_{1}, s_{1}, b_{1}^{\prime}}^{\mu_{0}}=\overline{a_{b_{1}, s_{1}, b_{1}^{\prime}}^{\mu_{0}}} \text {. }
$$

However, by the assumption $\mu_{0} \ngtr \lambda$ and $\mu_{0} \in P\left(\xi, \xi^{\prime}\right)$, we have $a_{b_{1}, s_{1}, b_{1}^{\prime}}^{\mu_{0}} \in q_{s} \mathbf{A}_{0}$ using Claim (i). Thus this equality is impossible unless $a_{b_{1}, s_{1}, b_{1}^{\prime}}^{\mu_{0}}=0$. This is a contradiction, and we get (a).

Similarly, if $\lambda \in P\left(\xi, \xi^{\prime}\right)$, we have $a_{b_{1}, s_{1}, b_{1}^{\prime}}^{\lambda}=\overline{a_{b_{1}, s_{1}, b_{1}^{\prime}}^{\lambda}}$. Then Claim (ii) implies $a_{b_{1}, s_{1}, b_{1}^{\prime}}^{\lambda}=\delta_{\left(b, s, b^{\prime}\right),\left(b_{1}, s_{1}, b_{1}^{\prime}\right)}$. We get $(\mathrm{b})$.

Now we have

Theorem 6.29. (i) $\widetilde{\mathbf{U}}[\lambda]$ has a crystal base $(\tilde{\mathcal{L}}[\lambda], \tilde{\mathcal{B}}[\lambda])$, where $\tilde{\mathcal{B}}[\lambda]$ is identified with a subset of $\tilde{\mathcal{L}}[\lambda] / q_{s} \tilde{\mathcal{L}}[\lambda]$ as in the proof of Proposition 6.23.

(ii) $\left(\tilde{\mathcal{L}}[\lambda], \overline{\tilde{\mathcal{L}}[\lambda]},{ }_{\mathcal{A}} \widetilde{\mathbf{U}}[\lambda]\right)$ is a balanced triple of $\widetilde{\mathbf{U}}[\lambda]$ and $\{G(b) \bmod \tilde{\mathbf{U}}[>\lambda] \mid b \in$ $\tilde{\mathcal{B}}[\lambda]\}$ is its global crystal basis.

Proof. By Proposition 6.27, we can take $G(\beta)\left(u_{\xi} \otimes u_{-\xi}\right)$ for the lift $\tilde{G}_{\lambda}\left(b, s, b^{\prime}\right)$ in Lemma 6.26. Therefore Lemma 6.26 implies that $\bigsqcup_{\mu \geq \lambda}\{G(\beta) \mid \beta \in \tilde{\mathcal{B}}[\mu]\}$ is a basis of $\widetilde{\mathbf{U}}[\geq \lambda]$. Hence $G(\tilde{\mathcal{B}}[\lambda]) \bmod \widetilde{\mathbf{U}}\left[{ }^{>} \lambda\right]$ is a basis of $\widetilde{\mathbf{U}}[\lambda]$. Other axioms of the global crystal basis obviously hold. 
6.2. Cell structure of $\widetilde{\mathbf{U}}$. We recall [24] the definition of cells in $\widetilde{\mathbf{U}}$ with respect to the global basis $G(\tilde{\mathcal{B}})=\{G(\beta) \mid \beta \in \mathcal{B}\}$. Let $\mathcal{F}$ be the collection of all subsets $K \subset \tilde{\mathcal{B}}$ with the property: the $\mathbf{Q}\left(q_{s}\right)$-subspace of $\widetilde{\mathbf{U}}$ spanned by $G(K)$ is a twosided ideal of $\widetilde{\mathbf{U}}$. If $\beta, \beta^{\prime} \in \tilde{\mathcal{B}}$, we say that $\beta \preceq \beta^{\prime}$ if $\beta \in \cap_{K \in \mathcal{F}, \beta^{\prime} \in K} K$. We say that $\beta \sim \beta^{\prime}$ if $\beta \preceq \beta^{\prime}$ and $\beta^{\prime} \preceq \beta$. The equivalence classes of $\sim$ are called two-sided cells. Similarly, by considering left ideals or right ideals above, we define $\preceq_{L}$ or $\preceq_{R}$. The equivalence classes are then called left cells or right cells respectively. Another equivalent definition of $\preceq$ is as follows: Let

$$
G(\beta) G\left(\beta^{\prime}\right)=\sum_{\beta^{\prime \prime}} c_{\beta \beta^{\prime}}^{\beta^{\prime \prime}}\left(q_{s}\right) G\left(\beta^{\prime \prime}\right)
$$

define the structure constants $c_{\beta \beta^{\prime}}^{\beta^{\prime \prime}}\left(q_{s}\right) \in \mathcal{A}$ of $\widetilde{\mathbf{U}}$ with respect to the global basis. For $\beta, \beta^{\prime} \in \tilde{\mathcal{B}}$ we say $\beta \preceq_{L} \beta^{\prime}$ (resp. $\preceq_{R}$ ) if there is a sequence $\beta_{1}=\beta^{\prime}, \beta_{2}, \ldots, \beta_{N}=\beta$ in $\tilde{\mathcal{B}}$ and a sequence $\gamma_{1}, \ldots, \gamma_{N-1} \in \tilde{\mathcal{B}}$ such that $c_{\gamma_{s}, \beta_{s}}^{\beta_{s+1}} \neq 0$ (resp. $c_{\beta_{s}, \gamma_{s}}^{\beta_{s+1}} \neq 0$ ) for $s=1, \ldots, N-1$. We write $\beta \preceq \beta^{\prime}$ if either of the above structure constants is non-zero for all $\beta_{s}, \gamma_{s}, \beta_{s+1}, s=1, \ldots, N-1$.

Proposition 6.30. (i) $\tilde{\mathcal{B}}[\lambda]$ is a two-sided cell of $\tilde{\mathcal{B}}$.

(ii) For any $b_{2} \in \mathcal{B}_{W}(\lambda),\left\{\left(b_{1}, s, b_{2}\right) \in \tilde{\mathcal{B}}[\lambda] \mid s \in \operatorname{Irr} G_{\lambda}, b_{1} \in \mathcal{B}_{W}(\lambda)\right\}$ is a left cell.

(iii) For any $b_{1} \in \mathcal{B}_{W}(\lambda),\left\{\left(b_{1}, s, b_{2}\right) \in \tilde{\mathcal{B}}[\lambda] \mid s \in \operatorname{Irr} G_{\lambda}, b_{2} \in \mathcal{B}_{W}(\lambda)\right\}$ is a right cell.

Proof. (i) Since $\widetilde{\mathbf{U}}\left[{ }^{\geq} \lambda\right], \widetilde{\mathbf{U}}\left[{ }^{>} \lambda\right]$ are two-sided ideals of $\widetilde{\mathbf{U}}, \tilde{\mathcal{B}}[\lambda]$ is a union of twosided cells. Let $b \in \tilde{\mathcal{B}}$. By $[17,6.4 .3], f_{i} G(\beta)$ is equal to $\left[\varepsilon_{i}(\beta)+1\right] G\left(\tilde{f}_{i} \beta\right)$ plus a linear combination of elements in $\tilde{\mathcal{B}}$ different from $\tilde{f}_{i} \beta$. Therefore $G\left(\tilde{f}_{i} \beta\right) \preceq_{L} G(\beta)$ if $\tilde{f}_{i} \beta \neq 0$. Similarly we have $G\left(\tilde{e}_{i} \beta\right) \preceq_{L} G(\beta)$ if $\tilde{e}_{i} \beta \neq 0$. Thus if $\beta$ and $\beta^{\prime}$ are in the same connected component of $\tilde{\mathcal{B}}$, we have $G(\beta) \sim_{L} G\left(\beta^{\prime}\right)$. Taking \#, we conclude that if $\beta$ and $\beta^{\prime}$ are in the same connected component of $\tilde{\mathcal{B}}$ as \#-crystal, we have $G(\beta) \sim_{R} G\left(\beta^{\prime}\right)$. By Theorem 4.16 we have the assertion if $a_{\lambda} \sim S a_{\lambda}$ for $s \in \operatorname{Irr} G_{\lambda}$. This follows from a consideration of two-sided cells of the based ring $\left(R\left(G_{\lambda}\right), \operatorname{Irr} G_{\lambda}\right)$, where $R\left(G_{\lambda}\right)$ is the representation $\operatorname{ring}$ of $G_{\lambda}$ and $\operatorname{Irr} G_{\lambda}$ is considered as its basis. By using the Pieri formula, we can easily check that it consists of a single two-sided cell, and hence our assertion. The proofs of (ii), (iii) are contained in the above proof.

$\operatorname{Remark} \tilde{\mathcal{B}}=\bigsqcup_{\lambda \in P_{\mathrm{cl},+}^{0}} \tilde{\mathcal{B}}[\lambda]$ by Lemma 6.25. Therefore the above proposition gives a complete description of two-sided, left, right cells of $\tilde{\mathcal{B}}$.

Next we define a function on $G(\tilde{\mathcal{B}})$ which will allow us construct a limit algebra $\widetilde{\mathbf{U}}_{0}=\bigoplus_{\lambda \in P_{\mathrm{cl},+}^{0}} \tilde{\mathbf{U}}[\lambda]_{0}$ as $q \rightarrow 0$.

Definition 6.31. Let $\beta=\left(b, s, b^{\prime}\right) \in \tilde{\mathcal{B}}[\lambda]$. Define $a(\beta)=-\left(\operatorname{wt}\left(b^{\prime}\right), 2 \lambda+\operatorname{wt}\left(b^{\prime}\right)\right) / 2$.

Remark 6.32. $a(\beta)$ is equal to the half of the dimension of the quiver variety $\mathfrak{M}(\mathbf{v}, \mathbf{w})$ (or the dimension of the lagrangian subvariety $\mathfrak{L}(\mathbf{v}, \mathbf{w})$ ), where $\mathbf{v}=$ $-\mathrm{wt}\left(b^{\prime}\right), \mathbf{w}=\lambda$. (See e.g. 29, 2.3.2(4)].) The inner product $($, ) induced on $P_{\mathrm{cl}}^{0}$ is equal to the standard inner product for the untwisted $A D E$ case (see 115 , Corollary 6.4]), which is the only case for which quiver varieties are defined. 
Lemma 6.33 (cf. [24, 1.4, 4.11]). Let $\beta=\left(b, s, b^{\prime}\right) \in \tilde{\mathcal{B}}[\lambda]$. The following holds:

(i) $\left(q^{a(\beta)} \beta\right) \tilde{\mathcal{L}}[\lambda] \subset \tilde{\mathcal{L}}[\lambda]$ and for each $\beta, a(\beta) \geq 0$ is smallest integer with this property.

(ii) for a two-sided cell $\tilde{\mathcal{B}}[\lambda]$ and any $\lambda_{1} \in P_{\mathrm{cl},+}^{0}$, the restriction of a to $\{\beta \in$ $\left.\tilde{\mathcal{B}}[\lambda] \mid G(\beta) \in \mathbf{U} a_{\lambda_{1}}\right\}$ is constant.

Proof. First note that using Lemmas 6.15, 6.17 and Proposition 6.27 we have the following equalities in $\widetilde{\mathbf{U}}[\lambda]=\widetilde{\mathbf{U}}[\geq \lambda] / \widetilde{\mathbf{U}}[>\lambda]$ :

$$
\begin{aligned}
& G\left(b, s, b^{\prime}\right) G\left(b_{1}, s_{1}, b_{1}^{\prime}\right) \bmod \widetilde{\mathbf{U}}\left[^{>} \lambda\right] \\
= & q^{-a(\beta)} \sum_{s^{\prime} \in \operatorname{Irr} G_{\lambda}}\left(G\left(b_{1}\right) S_{1} u_{\lambda}, G\left(b^{\prime}\right) S^{\prime} u_{\lambda}\right)_{\lambda} G(b) S S^{\prime} G\left(b_{1}^{\prime}\right)^{\#} \bmod \widetilde{\mathbf{U}}\left[^{>} \lambda\right] \\
= & q^{-a(\beta)} \sum_{s^{\prime}, s^{\prime \prime} \in \operatorname{Irr} G_{\lambda}} c_{s, s^{\prime}}^{s^{\prime \prime}}\left(G\left(b_{1}\right) S_{1} u_{\lambda}, G\left(b^{\prime}\right) S^{\prime} u_{\lambda}\right)_{\lambda} G(b) S^{\prime \prime} G\left(b_{1}^{\prime}\right)^{\#} \bmod \widetilde{\mathbf{U}}\left[^{>} \lambda\right]
\end{aligned}
$$

where $s s^{\prime}=\sum_{s^{\prime \prime}} c_{s s_{\tilde{s}}^{\prime \prime}}^{s^{\prime \prime}} s^{\prime \prime}$ and $c_{s s^{\prime}}^{s^{\prime \prime}} \in \mathbf{Z}_{\geq 0}$. By Theorem 2.20 (and Lemma 6.14) this is contained in $q^{-a(\beta)} \tilde{\mathcal{L}}[\lambda]$. (i) now follows by multiplying both sides by $q^{a(\beta)}$. Note that when $b_{1}^{\prime}=b_{2}$ the product is in $\tilde{\mathcal{L}}[\lambda] \backslash q_{s} \tilde{\mathcal{L}}[\lambda]$ by Theorem 2.20 and so $a(\beta)$ is the smallest integer with this property. We check that $a(\beta)$ is positive. We have $G\left(b, s, b^{\prime}\right) G\left(b^{\prime}, 1, b\right)=q^{-a(\beta)} G(b, s, b) \bmod \left(\tilde{\mathcal{L}}[>\lambda]+q_{s} \tilde{\mathcal{L}}[\geq \lambda]\right)$ by Theorem 2.20 . It follows $c\left(q_{s}\right)=c_{\beta,\left(b^{\prime}, 1, b\right)}^{(b, s)}\left(q_{s}\right)=q^{-a(\beta)} \bmod q_{s} \mathbf{Z}\left[q_{s}\right]$. Taking - of both sides and using the ${ }^{-}$invariance of the global basis we also have $c\left(q_{s}\right)=q^{a(\beta)} \bmod q_{s}^{-1} \mathbf{Z}\left[q_{s}^{-1}\right]$. This implies $a(\beta) \geq 0$. (ii) is clear from the definition: If $\beta=\left(b, s, b^{\prime}\right) \in \tilde{\mathcal{B}}[\lambda]$ satisfies $G(\beta) \in \widetilde{\mathbf{U}} a_{\lambda_{1}}$, we have wt $\left(b^{\prime}\right)=\lambda_{1}-\lambda$.

Using Lemma 6.33 for any two-sided cell, we define the ring $\widetilde{\mathbf{U}}[\lambda]_{0}$ which is a "limit as $q \rightarrow 0$." For $\beta \in \tilde{\mathcal{B}}[\lambda]$ define $\hat{\beta}=q^{a(\beta)} G(\beta)$. Then $\{\hat{\beta} \mid \beta \in \tilde{\mathcal{B}}[\lambda]\}$ is a $\mathbf{Q}\left(q_{s}\right)$-basis of $\tilde{\mathbf{U}}[\lambda]$. For $\beta, \beta^{\prime} \in \tilde{\mathcal{B}}[\lambda]$ such that $G\left(\beta^{\prime}\right) \in \mathbf{U} a_{\lambda_{1}}$, we have $\hat{\beta} \hat{\beta}^{\prime}=$ $\sum_{\hat{\beta}^{\prime \prime}} q^{a(\beta)} c_{\beta, \beta^{\prime}}^{\beta^{\prime \prime}} \hat{\beta}^{\prime \prime}$ in $\widetilde{\mathbf{U}}[\lambda]$. (This follows from $a\left(\beta^{\prime}\right)=a\left(\beta^{\prime \prime}\right)$, which follows from Lemma 6.33(ii) above, since the only non-zero coefficients $c_{\beta, \beta^{\prime}}^{\beta^{\prime \prime}}$ are those for which $\left.G\left(\beta^{\prime \prime}\right) \in \mathbf{U} a_{\lambda_{1}}\right)$. Since $q^{a(\beta)} c_{\beta, \beta^{\prime}}^{\beta^{\prime \prime}} \in \mathbf{Z}\left[q_{s}\right]$ by Lemma $6.33(\mathrm{i})$, the $\mathbf{Z}\left[q_{s}\right]$-submodule $\widetilde{\mathbf{U}}[\lambda]^{-}$of $\tilde{\mathbf{U}}[\lambda]$ generated by $\{\hat{\beta} \mid \beta \in G(\tilde{\mathcal{B}}[\lambda])\}$ is a $\mathbf{Z}\left[q_{s}\right]$-subalgebra of $\widetilde{\mathbf{U}}[\lambda]$. We define $\widetilde{\mathbf{U}}[\lambda]_{0}=\widetilde{\mathbf{U}}[\lambda]^{-} / q_{s} \widetilde{\mathbf{U}}[\lambda]^{-}$. Define $t_{\beta}$ to be the image of $\hat{\beta}$ in $\widetilde{\mathbf{U}}[\lambda]_{0}$. Then $\widetilde{\mathbf{U}}[\lambda]_{0}$ is a ring with a $\mathbf{Z}$-basis $\left\{t_{\beta} \mid \beta \in G(\tilde{\mathcal{B}}[\lambda])\right\}$. If we denote by $\left\{\gamma_{\beta, \beta^{\prime}}^{\beta^{\prime \prime}} \mid \beta, \beta^{\prime}, \beta^{\prime \prime} \in\right.$ $G(\tilde{\mathcal{B}}[\lambda])\}$ the structure constants of $\widetilde{\mathbf{U}}[\lambda]_{0}$ then $q^{a(\beta)} c_{\beta, \beta^{\prime}}^{\beta^{\prime \prime}} \equiv \gamma_{\beta, \beta^{\prime}}^{\beta^{\prime \prime}} \bmod q_{s} \mathbf{Z}\left[q_{s}\right]$. We define $\widetilde{\mathbf{U}}_{0}=\bigoplus \widetilde{\mathbf{U}}[\lambda]_{0}$ to be the direct sum of these rings.

Lemma 6.34. $G\left(b, s, b^{\prime}\right)^{\#}=G\left(b^{\prime}, s^{\#}, b\right)$ for $\left(b, s, b^{\prime}\right) \in \tilde{\mathcal{B}}[\lambda]$.

Proof. Note $\left(G(b) S G\left(b^{\prime}\right)^{\#}\right)^{\#}=G\left(b^{\prime}\right) S^{\#} G(b)^{\#}$. By Lemma $6.13 S^{\#}$ corresponds to the dual representation $s^{\#}$ modulo $\widetilde{\mathbf{U}}[\lambda]$. We have $G_{\lambda}\left(b, s, b^{\prime}\right)^{\#}=G_{\lambda}\left(b^{\prime}, s^{\#}, b\right)$ by the definition, then the assertion follows by Proposition 6.27.

Definition 6.35. Let $\lambda \in P_{\mathrm{cl},+}^{0}$. Let $\mathcal{D}_{\tilde{\mathcal{B}}[\lambda]}=\left\{(b, 1, b) \mid b \in \mathcal{B}_{W}(\lambda)\right\}$. Denote by $\widetilde{\mathbf{U}}(0)$ the subalgebra of $\widetilde{\mathbf{U}}$ generated by $a_{\lambda} x a_{\lambda}$ for $x \in \mathbf{U}$.

Remarks 6.36. (i) By the definition and Lemma 6.34, it is clear that $G(\beta)^{\#}=$ $G(\beta)$ for $\beta \in \mathcal{D}_{\tilde{\mathcal{B}}[\lambda]}$. It is also clear that $G(\beta)$ is in $\widetilde{\mathbf{U}}(0)$. 
(ii) It is not true that $G(\beta)^{\#}=G(\beta)$ implies $\beta \in \mathcal{D}_{\tilde{\mathcal{B}}[\lambda]}$. Consider $(b, s, b)$ with $s^{\#}=s$ and $s \neq 1$, e.g. $s(z)=\left(z_{1}^{2}+z_{1} z_{2}+z_{2}^{2}\right) / z_{1} z_{2}$.

(iii) The identification (6.4) depends on the choice of the section $\mathcal{B}_{W}(\lambda) \rightarrow$ $\mathcal{B}_{0}(\lambda)$. But the ambiguity of monomials $p(z)=\prod_{i}\left(z_{i, 1} \cdots z_{i, \lambda_{i}}\right)^{r_{i}}$ cancels in $G(\beta)=$ $G(b, 1, b)$. Therefore the bijection $\mathcal{B}_{W}(\lambda) \rightarrow \mathcal{D}_{\tilde{\mathcal{B}}[\lambda]}$ is independent of the section.

The following gives a characterization of $\mathcal{D}_{\tilde{\mathcal{B}}[\lambda]}$ :

Proposition 6.37 (cf. [24, Conj. 5.3(b)]). Let $\lambda_{1} \in P_{\mathrm{cl}}^{0}$. If $G(\beta) \in \widetilde{\mathbf{U}}(0) a_{\lambda_{1}} \cap$ $G(\tilde{\mathcal{B}}[\lambda])$, then $q^{-a(\beta)}\left(a_{\lambda_{1}}, G(\beta)\right) \equiv 1 \bmod q_{s} \mathbf{Z}\left[q_{s}\right]$ if $\beta \in \mathcal{D}_{\tilde{\mathcal{B}}[\lambda]}$ and $\equiv 0$ otherwise.

Proof. By definition, $a$ takes the constant $\left(\lambda-\lambda_{1}, \lambda+\lambda_{1}\right) / 2$ on $\widetilde{\mathbf{U}}(0) a_{\lambda_{1}} \cap G(\tilde{\mathcal{B}}[\lambda])$. We denote this constant by $a_{0}$. If $\mu>\lambda$, then

$$
\left(\mu-\lambda_{1}, \mu+\lambda_{1}\right)-\left(\lambda-\lambda_{1}, \lambda+\lambda_{1}\right)=(\mu-\lambda, \mu+\lambda)>0 .
$$

Thus our assertion is equivalent to saying that if $G(\beta) \in \widetilde{\mathbf{U}}(0) a_{\lambda_{1}} \cap \bigsqcup_{\mu \geq \lambda} G(\tilde{\mathcal{B}}[\mu])$, then $q^{-a_{0}}\left(a_{\lambda_{1}}, G(\beta)\right) \equiv 1 \bmod q_{s} \mathbf{Z}\left[q_{s}\right]$ if $\beta \in \mathcal{D}_{\tilde{\mathcal{B}}[\lambda]}$ and $\equiv 0$ otherwise. We will check this.

In this proof we replace $\widetilde{\mathbf{U}}$ by $\bigoplus_{\lambda \in P} \mathbf{U} a_{\lambda}$ the modified quantum enveloping algebra defined for $P$. Then by [23, 26.2.3], ( , ) is the limit of the inner product on $V(\xi) \otimes V\left(-\xi^{\prime}\right)$ (denoted also by $\left.(),\right)$, where $\xi-\xi^{\prime}=\lambda_{1}$ and $\xi, \xi^{\prime}$ tend to $\infty$.

For each $G(\beta)=G\left(b, s, b^{\prime}\right) \in \widetilde{\mathbf{U}}(0) a_{\lambda_{1}} \cap \bigsqcup_{\mu \geq \lambda} G(\tilde{\mathcal{B}}[\mu])$, we choose and fix an expression $(b, s)=X_{1} X_{2} \cdots X_{N}\left(u_{\lambda}, s_{0}\right)$ where $s_{0} \in \operatorname{Irr} G_{\lambda}, X_{i}=\tilde{e}_{i}$ or $\tilde{f}_{i}$. Then

$$
\begin{aligned}
& q^{-a(\beta)}\left(u_{\xi} \otimes u_{-\xi^{\prime}}, X_{1} X_{2} \cdots X_{N} S_{0} G\left(b^{\prime}\right)^{\#}\left(u_{\xi} \otimes u_{-\xi^{\prime}}\right)\right) \\
= & \left(\left(X_{1} X_{2} \cdots X_{N} S_{0}\right)^{\#}\left(u_{\xi} \otimes u_{-\xi^{\prime}}\right), G\left(b^{\prime}\right)^{\#}\left(u_{\xi} \otimes u_{-\xi^{\prime}}\right)\right) \\
\equiv & \left(G(\tilde{b})\left(u_{\xi} \otimes u_{-\xi^{\prime}}\right), G\left(b^{\prime}\right)^{\#}\left(u_{\xi} \otimes u_{-\xi^{\prime}}\right)\right) \quad \bmod q_{s} \mathbf{Z}\left[q_{s}\right] \\
\equiv & \delta_{(b, s),\left(b^{\prime}, 1\right)} \quad \bmod q_{s} \mathbf{Z}\left[q_{s}\right],
\end{aligned}
$$

where $\tilde{b}=(b, s)$ by $(6.4)$. Here we have used $(6.3)$ in the first equality, and the almost orthonomal property of the global crystal basis $(23,26.3 .1])$ in the second and third equalities. Since $\left\{G(\beta)=G\left(b, s, b^{\prime}\right)\right\}=\widetilde{\mathbf{U}}(0) a_{\lambda_{1}} \cap \bigsqcup_{\mu \geq \lambda} G(\tilde{\mathcal{B}}[\mu])$ is a $\mathbf{A}_{0^{-}}$ basis of $\tilde{\mathcal{L}}[\geq \lambda]$, the set of corresponding elements $\left\{X_{1} X_{2} \cdots X_{N} S_{0} G\left(b^{\prime}\right)^{\#}\left(u_{\xi} \otimes u_{-\xi^{\prime}}\right)\right\}$ spans $\left(\tilde{\mathbf{U}}(0) a_{\lambda_{1}} \cap \tilde{\mathcal{L}}(\geq \lambda)\right)\left(u_{\xi} \otimes u_{-\xi^{\prime}}\right)$. Therefore the above together with our previous remark $a(\beta) \geq a_{0}$ implies

$$
q^{-a_{0}}\left(u_{\xi} \otimes u_{-\xi^{\prime}},\left(\widetilde{\mathbf{U}}(0) a_{\lambda_{1}} \cap \tilde{\mathcal{L}}(\geq \lambda)\right)\left(u_{\xi} \otimes u_{-\xi^{\prime}}\right)\right) \in \mathbf{Z}\left[q_{s}\right] .
$$

Since $G\left(b, s, b^{\prime}\right)\left(u_{\xi} \otimes u_{-\xi^{\prime}}\right) \equiv X_{1} X_{2} \cdots X_{N} S_{0} G\left(b^{\prime}\right)^{\#}\left(u_{\xi} \otimes u_{-\xi^{\prime}}\right) \bmod \tilde{\mathcal{L}}\left[{ }^{\geq}\right]\left(u_{\xi} \otimes\right.$ $\left.u_{-\xi^{\prime}}\right)$, the above shows

$$
q^{-a_{0}}\left(u_{\xi} \otimes u_{-\xi^{\prime}}, G\left(b, s, b^{\prime}\right)\left(u_{\xi} \otimes u_{-\xi^{\prime}}\right)\right) \equiv \delta_{\lambda, \mu} \delta_{(b, s),\left(b^{\prime}, 1\right)} \quad \bmod q_{s} \mathbf{Z}\left[q_{s}\right] .
$$

Taking limit $\xi, \xi^{\prime} \rightarrow \infty$, we get the assertion.

Lemma 6.38 (cf. 24, 1.5]). Let $d_{1}=G\left(b_{1}, 1, b_{1}\right), d_{2}=G\left(b_{2}, 1, b_{2}\right)$ be in $\mathcal{D}_{\tilde{\mathcal{B}}[\lambda]}$. Let $\beta=G\left(b_{3}, s, b_{3}^{\prime}\right)$. Then in $\widetilde{\mathbf{U}}[\lambda]_{0}, t_{d_{1}} t_{\beta} t_{d_{2}}=\delta_{b_{1}, b_{3}} \delta_{b_{3}^{\prime}, b_{2}} t_{\beta}$. 
Proof. By Lemma 6.17 we have

$$
\begin{aligned}
G\left(b_{1}, 1, b_{1}\right) & G\left(b_{3}, s, b_{3}^{\prime}\right) \\
& \equiv q^{-a\left(d_{1}\right)} \sum_{s^{\prime \prime} \in \operatorname{Irr} G_{\lambda}}\left(G\left(b_{3}\right) S u_{\lambda}, G\left(b_{1}\right) S^{\prime \prime} u_{\lambda}\right)_{\lambda} G\left(b_{1}\right) S^{\prime \prime} G\left(b_{3}^{\prime}\right)^{\#} \bmod \tilde{\mathcal{L}}[>\lambda] \\
& \equiv q^{-a\left(d_{1}\right)} \delta_{b_{1}, b_{3}} G\left(b_{1}\right) S G\left(b_{3}^{\prime}\right)^{\#} \bmod \left(\tilde{\mathcal{L}}\left[{ }^{>} \lambda\right]+q_{s} \tilde{\mathcal{L}}\left[{ }^{\geq} \lambda\right]\right) .
\end{aligned}
$$

The second equivalence follows from Theorem 2.20. A similar calculation shows

$$
G_{\lambda}\left(b_{3}, s, b_{3}^{\prime}\right) G_{\lambda}\left(b_{2}, 1, b_{2}\right) \equiv q^{-a(\beta)} \delta_{b_{3}^{\prime}, b_{2}} G\left(b_{3}, s, b_{3}^{\prime}\right) \bmod \left(\tilde{\mathcal{L}}[>\lambda]+q_{s} \tilde{\mathcal{L}}[\geq \lambda]\right) .
$$

Since $a(\beta)=a\left(d_{2}\right)$ if $b_{3}^{\prime}=b_{2}$ the result follows by multiplying both sides by $q^{2 a\left(d_{2}\right)}$.

Remark 6.39. Lemma 6.38 says that the $\mathbf{Z}$-ring $\widetilde{\mathbf{U}}[\lambda]_{0}$ has a generalized unit which is compatible with the basis $t_{\beta}, \beta \in G(\tilde{\mathcal{B}}[\lambda])$. In particular, the ring $\widetilde{\mathbf{U}}[\lambda]_{0}$ has an identity, $1=\sum_{b_{1} \in B_{W}(\lambda)} t_{G\left(b_{1}, 1, b_{1}\right)}$.

$\widetilde{\mathbf{U}}[\lambda]$ is both a left $\widetilde{\mathbf{U}}$-module and a right $\widetilde{\mathbf{U}}$-module where the respective module structures are given by:

$$
G(\beta) G\left(\beta^{\prime}\right)=\sum_{\beta^{\prime \prime} \in \tilde{\mathcal{B}}[\lambda]} c_{\beta \beta^{\prime}}^{\beta^{\prime \prime}}\left(q_{s}\right) G\left(\beta^{\prime \prime}\right),
$$

when $\beta \in \tilde{\mathcal{B}}, \beta^{\prime} \in \tilde{\mathcal{B}}[\lambda]$ in the first case and when $\beta \in \tilde{\mathcal{B}}[\lambda], \beta^{\prime} \in \tilde{\mathcal{B}}$ in the second case.

We define $M_{\widetilde{\mathbf{U}}[\lambda]}$ to be the vector space spanned over $\mathbf{Q}\left(q_{s}, q_{s}^{\prime}\right)$ by $\{G(\beta) \mid \beta \in$ $\tilde{\mathcal{B}}[\lambda]\} . \quad M_{\widetilde{\mathbf{U}}[\lambda]}$ is a $\widetilde{\mathbf{U}}$-bimodule where the left action is given by $(6.40$ and the right action is given by (6.40) where $c_{\beta \beta^{\prime}}^{\beta^{\prime \prime}}\left(q_{s}\right)$ is replace by $c_{\beta \beta^{\prime}}^{\beta^{\prime \prime}}\left(q_{s}^{\prime}\right)$. We now show that the left and right module structures commute, i.e., $G\left(\beta_{1}\right)\left(G\left(\beta_{2}\right) G\left(\beta_{3}\right)\right)=$ $\left(G\left(\beta_{1}\right) G\left(\beta_{2}\right)\right) G\left(\beta_{3}\right)$ where $\beta_{2} \in \tilde{\mathcal{B}}[\lambda]$ and $\beta_{1}, \beta_{3} \in \tilde{\mathcal{B}}$. In terms of the structure constants this is equivalent to

Lemma 6.41 (cf. 24] 1.7, 4.15]). Let $\beta_{4}, \beta_{2} \in \tilde{\mathcal{B}}[\lambda]$. Let $\beta_{1}, \beta_{3} \in \tilde{\mathcal{B}}$. Then

$$
\sum_{\beta \in \tilde{\mathcal{B}}[\lambda]} c_{\beta_{1}, \beta}^{\beta_{4}}\left(q_{s}\right) c_{\beta_{2}, \beta_{3}}^{\beta}\left(q_{s}^{\prime}\right)=\sum_{\beta \in \tilde{\mathcal{B}}[\lambda]} c_{\beta_{1}, \beta_{2}}^{\beta}\left(q_{s}\right) c_{\beta, \beta_{3}}^{\beta_{4}}\left(q_{s}^{\prime}\right) .
$$

Proof. We fix $\beta_{i}=\left(b_{i}, s_{i}, b_{i}^{\prime}\right)$ for $i=2,4$. For $\beta_{j} \in \tilde{\mathcal{B}}(j=1,3)$ and $b_{i} \in B_{W}(\lambda)$ $(i=2,4)$, we define $g_{\beta_{j}, b_{i}}^{(s, b)} \in \mathcal{A}$ by

$$
G\left(\beta_{j}\right) G_{\lambda}\left(1, b_{i}\right)=\sum_{(s, b) \in \mathcal{B}(\lambda)} g_{\beta_{j}, b_{i}}^{(s, b)} G_{\lambda}(s, b) .
$$

We check that

(a) $G\left(\beta_{j}\right) G\left(b_{i}, s_{i}, b_{i}^{\prime}\right) \equiv \sum_{(s, b) \in \mathcal{B}(\lambda)} \sum_{s^{\prime} \in \operatorname{Irr} G_{\lambda}} c_{s s_{i}}^{s^{\prime}} g_{\beta_{j}, b_{i}}^{(s, b)} G\left(b, s^{\prime}, b_{i}^{\prime}\right) \bmod \widetilde{\mathbf{U}}\left[{ }^{>} \lambda\right]$,

(b) $G\left(b_{i}, s_{i}, b_{i}^{\prime}\right) G\left(\beta_{j}\right) \equiv \sum_{(s, b) \in \mathcal{B}(\lambda)} \sum_{s^{\prime} \in \operatorname{Irr} G_{\lambda}} c_{s s_{i}^{\#}}^{s^{\prime \#}} g_{\beta_{j}^{\#}, b_{i}^{\prime}}^{(s, b)} G\left(b_{i}, s^{\prime}, b\right) \bmod \tilde{\mathbf{U}}\left[{ }^{>} \lambda\right]$, 
where $c_{\bullet \bullet}$ are structure constants of $\left(R\left(G_{\lambda}\right), \operatorname{Irr} G_{\lambda}\right)$ as before.

We consider the $\widetilde{\mathbf{U}}$-homomorphism $\Psi_{\tilde{b}_{i}}: V(\lambda) \rightarrow \widetilde{\mathbf{U}}[\lambda]$ such that $\Psi_{\tilde{b}_{i}}\left(u_{\lambda}\right)=$ $S_{i} G\left(b_{i}^{\prime}\right)^{\#} \bmod \widetilde{\mathbf{U}}[>\lambda]$, where $\tilde{b}_{i}=\left(s_{i}^{\#}, b_{i}^{\prime}\right)$ by 6.4$)$. (The existence of $\Psi_{\tilde{b}_{i}}$ is guaranteed by Lemma 6.11.) The image of (6.42) under $\Psi_{\tilde{b}_{i}}$ gives

$$
G\left(\beta_{j}\right) G\left(b_{i}, s_{i}, b_{i}^{\prime}\right) \equiv \sum_{(s, b)} g_{\beta_{j}, b_{i}}^{(s, b)} G(b) S S_{i} G\left(b_{i}^{\prime}\right)^{\#} .
$$

Now (a) follows. (b) follows from (a) by taking \#.

By (a) and (b) we have

$$
\begin{aligned}
& c_{\beta_{1}, \beta_{2}}^{\beta}\left(q_{s}\right)=\sum_{s^{\prime}} \delta_{b_{2}^{\prime}, b^{\prime}} c_{s^{\prime} s_{2}}^{s} g_{\beta_{1}, b_{2}}^{\left(s^{\prime}, b\right)}\left(q_{s}\right), \\
& c_{\beta, \beta_{3}}^{\beta_{4}}\left(q_{s}^{\prime}\right)=\sum_{s^{\prime}} \delta_{b, b_{4}} c_{s^{\prime} s^{\#}}^{s_{\#}^{\#}} g_{\beta_{3}^{\#}, b^{\prime}}^{\left(s^{\prime}, b^{\prime}\right)}\left(q_{s}^{\prime}\right), \\
& c_{\beta_{1}, \beta}^{\beta_{4}}\left(q_{s}\right)=\sum_{s^{\prime}} \delta_{b_{4}^{\prime}, b^{\prime}} c_{s^{\prime} s}^{s_{4}} g_{\beta_{1}, b}^{\left(s^{\prime}, b_{4}\right)}\left(q_{s}\right), \\
& c_{\beta_{2}, \beta_{3}}^{\beta}\left(q_{s}^{\prime}\right)=\sum_{s^{\prime}} \delta_{b, b_{2}} c_{s^{\prime} s_{2}^{\#}}^{s^{\#}} g_{\beta_{3}^{\#}, b_{2}^{\prime}}^{\left(s^{\prime}, b^{\prime}\right)}\left(q_{s}^{\prime}\right),
\end{aligned}
$$

where $\beta=G\left(b, s, b^{\prime}\right)$. This makes the identity of the lemma equivalent to

$$
\begin{aligned}
\sum_{\left(b, s, b^{\prime}\right) \in \tilde{\mathcal{B}}[\lambda]} \sum_{s^{\prime}, s^{\prime \prime} \in \operatorname{Irr} G_{\lambda}} \delta_{b_{2}^{\prime}, b^{\prime}} c_{s^{\prime} s_{2}}^{s} g_{\beta_{1}, b_{2}}^{\left(s^{\prime}, b\right)}\left(q_{s}\right) \delta_{b, b_{4}} c_{s^{\prime \prime} s}^{s_{4}^{\#}} g_{\beta_{3}^{\#}, b^{\prime}}^{\left(s^{\prime \prime}, b_{4}^{\prime}\right)}\left(q_{s}^{\prime}\right) \\
=\sum_{\left(b, s, b^{\prime}\right) \in \tilde{\mathcal{B}}[\lambda]} \sum_{s^{\prime}, s^{\prime \prime} \in \operatorname{Irr} G_{\lambda}} \delta_{b_{4}^{\prime}, b^{\prime}} c_{s^{\prime} s}^{s_{4}} g_{\beta_{1}, b}^{\left(s^{\prime}, b_{4}\right)}\left(q_{s}\right) \delta_{b, b_{2}} c_{s^{\prime \prime} s_{2}^{\#}}^{s^{\#}} g_{\beta_{3}^{\#}, b_{2}^{\prime}}^{\left(s^{\prime \prime}, b^{\prime}\right)}\left(q_{s}^{\prime}\right) .
\end{aligned}
$$

Note that

$$
\sum_{s} c_{s^{\prime} s_{2}}^{s} c_{s^{\prime \prime} s^{\#}}^{s_{4}^{\#}}=\sum_{s} c_{s^{\prime} s_{2}}^{s} c_{s^{\prime \prime \#} s}^{s_{4}} \quad \text { and } \quad \sum_{s} c_{s^{\prime} s}^{s_{4}} c_{s^{\prime \prime} s_{2}^{\#}}^{s^{\#}}=\sum_{s} c_{s^{\prime} s}^{s_{4}} c_{s^{\prime \prime} \# s_{2}}^{s}
$$

are equal, since both are the multiplicity of $s_{4}$ in $s^{\prime} \otimes s_{2} \otimes s^{\prime \prime \#}$. Now the above identity is immediate.

Fix a two-sided cell $G(\tilde{\mathcal{B}}[\lambda])$. We define a $\mathbf{Q}\left(q_{s}\right)$-linear map $\Phi: \widetilde{\mathbf{U}} \rightarrow \mathbf{Q}\left(q_{s}\right) \otimes$ $\widetilde{\mathbf{U}}[\lambda]_{0}$ by

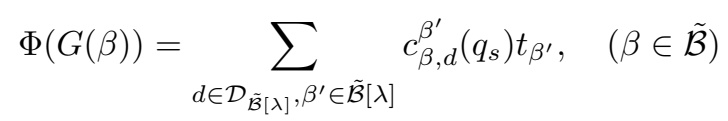

which is well-defined since $\mathcal{D}_{\tilde{\mathcal{B}}[\lambda]}$ is finite and for a fixed $\beta, d$ there are only finitely $\operatorname{many} c_{\beta, d}^{\beta^{\prime}} \neq 0$.

We have the following description of $\Phi$ due to [24, Prop. 1.9]

Proposition 6.43. (i) $\Phi$ is an algebra homomorphism.

(ii) Let $P(\tilde{\mathcal{B}}[\lambda])$ be the set of $\lambda \in P$ such that $a_{\lambda} d=d$ for some $d \in \mathcal{D}_{\tilde{\mathcal{B}}[\lambda]}$. Then $\Phi\left(\sum_{\lambda \in P(\tilde{\mathcal{B}}[\lambda])} a_{\lambda}\right)=1$, and $\Phi\left(a_{\lambda^{\prime}}\right)=0$ for $\lambda \notin P(\tilde{\mathcal{B}}[\lambda])$.

Next we describe an explicit realization of the ring structure of $\widetilde{\mathbf{U}}[\lambda]_{0}$. As usual, $\lambda=\sum_{i} \lambda_{i} \varpi_{i}$. Let $T_{\lambda}$ be the set of triples $\left(d, d^{\prime}, s\right)$ where $d, d^{\prime} \in D_{\tilde{\mathcal{B}}[\lambda]}$ and $s \in \operatorname{Irr} G_{\lambda}$. 
Let $J_{\lambda}$ be the free abelian group on $T_{\lambda}$ with a ring structure defined by

$$
\left(d_{1}, d_{1}^{\prime}, s\right)\left(d_{2}, d_{2}^{\prime}, s^{\prime}\right)=\delta_{d_{1}^{\prime}, d_{2}} \sum_{s^{\prime \prime} \in \operatorname{Irr} G_{\lambda}} c_{s s^{\prime}}^{s^{\prime \prime}}\left(d_{1}, d_{2}^{\prime}, s^{\prime \prime}\right),
$$

where $c_{s s^{\prime}}^{s^{\prime \prime}}$ is the multiplicity of $s^{\prime \prime}$ in the tensor product $s \otimes s^{\prime}$ as above. We have:

Theorem 6.44. (i) There exists a ring isomorphism $\widetilde{\mathbf{U}}[\lambda]_{0} \stackrel{\sim}{\longrightarrow} J_{\lambda}$ which gives a bijection between $\left\{t_{\beta} \mid \beta \in \tilde{\mathcal{B}}[\lambda]\right\}$ and $\left\{\left(d, d^{\prime}, s\right) \mid d, d^{\prime} \in \mathcal{D}_{\tilde{\mathcal{B}}[\lambda]}, s \in \operatorname{Irr} G_{\lambda}\right\}$.

(ii) For any $d_{0} \in \mathcal{D}_{\tilde{\mathcal{B}}[\lambda]}$, the subset of $\tilde{\mathcal{B}}[\lambda]$ corresponding to $\left\{\left(d, d^{\prime}, s\right) \in T_{\lambda} \mid d^{\prime}=\right.$ $\left.d_{0}\right\}$ under the bijection in (i) is a left cell.

(iii) For any $d_{0} \in \mathcal{D}_{\tilde{\mathcal{B}}[\lambda]}$, the subset of $\tilde{\mathcal{B}}[\lambda]$ corresponding to $\left\{\left(d, d^{\prime}, s\right) \in T_{\lambda} \mid d=\right.$ $\left.d_{0}\right\}$ under the bijection in (i) is a right cell.

Proof. By definition, the elements $b \in \mathcal{B}_{W}(\lambda)$ are in 1-1 correspondence with the elements of $t_{b}=t_{(b, 1, b)} \in D_{\tilde{\mathcal{\Omega}}[\lambda]}$. The map which sends $t_{\left(b_{1}, s, b_{1}^{\prime}\right)} \mapsto\left(t_{b_{1}}, t_{b_{1}^{\prime}}, s\right)$ is a bijection. Using Lemma 6.17 as in the proof of Lemma 6.33 we have

$$
t_{\left(b_{1}, s, b_{2}\right)} t_{\left(b_{1}^{\prime}, s^{\prime}, b_{2}^{\prime}\right)}=\delta_{b_{2}, b_{1}^{\prime}} \sum_{s_{1} \in \operatorname{Irr} G_{\lambda}} c_{s, s^{\prime}}^{s_{1}} t_{\left(b_{1}, s_{1}, b_{2}^{\prime}\right)} .
$$

This implies (i). (ii) and (iii) follow from Proposition 6.30.

The following proposition partly explains why our approach to the limit algebra $J_{\lambda}$ via $V(\lambda)$ is natural.

Proposition 6.46. Let $V(\lambda)_{0}$ be the free $\mathbf{Z}$-module with basis $\mathcal{B}(\lambda) \cong \mathcal{D}_{\tilde{\mathcal{B}}[\lambda]} \times \operatorname{Irr} G_{\lambda}$ endowed with a $J_{\lambda}$-module structure by

$$
\left(d_{1}, d_{2}, s\right) \cdot\left(d^{\prime}, s^{\prime}\right)=\delta_{d_{2}, d^{\prime}} \sum_{s^{\prime \prime} \in \operatorname{Irr} G_{\lambda}} c_{s s^{\prime}}^{s^{\prime \prime}}\left(d_{1}, s^{\prime \prime}\right) .
$$

Then $V(\lambda)_{0} \otimes_{\mathbf{Z}} \mathbf{Q}\left(q_{s}\right)$, pulled back by the composition of $\widetilde{\mathbf{U}} \stackrel{\Phi}{\rightarrow} \widetilde{\mathbf{U}}[\lambda]_{0} \stackrel{\sim}{\longrightarrow} J_{\lambda}$, is isomorphic to $V(\lambda)$.

Proof. For $b=\left(b_{W}, s\right) \in \mathcal{B}(\lambda) \cong \mathcal{B}_{W}(\lambda) \times \operatorname{Irr} G_{\lambda}$, we define $\beta_{b} \in \tilde{\mathcal{B}}[\lambda]$ by $\beta_{b}=$ $\left(b_{W}, s, u_{\lambda}\right)$. For $\beta \in \tilde{\mathcal{B}}$, we have

$$
G(\beta) G_{\lambda}(b)=\sum_{b^{\prime} \in \mathcal{B}(\lambda)} c_{\beta \beta_{b}}^{\beta_{b^{\prime}}} G_{\lambda}\left(b^{\prime}\right) .
$$

In fact, $G_{\lambda}(b)=G\left(\beta_{b}\right) u_{\lambda}$ implies $G(\beta) G_{\lambda}(b)=\sum_{\beta^{\prime} \in \tilde{\mathcal{B}}} c_{\beta \beta_{b}}^{\beta^{\prime}} G\left(\beta^{\prime}\right) u_{\lambda}$ and $G\left(\beta^{\prime}\right) u_{\lambda}$ is $G_{\lambda}\left(\beta^{\prime}\right)$ if $\beta^{\prime} \in \mathcal{B}(\lambda)$ and 0 otherwise. On the other hand, the $\widetilde{\mathbf{U}}[\lambda]_{0}$-module structure on $V(\lambda)_{0}$ is given by

$$
t_{\beta^{\prime}} b=\sum_{b^{\prime} \in \mathcal{B}(\lambda)} \gamma_{\beta^{\prime} \beta_{b}}^{\beta_{b^{\prime}}} b^{\prime} \quad \text { for } \beta^{\prime} \in \tilde{\mathcal{B}}[\lambda] .
$$

We define a $\mathbf{Q}\left(q_{s}\right)$-linear map $\Psi: V(\lambda) \rightarrow V(\lambda)_{0} \otimes \mathbf{z} \mathbf{Q}\left(q_{s}\right)$ by

$$
\Psi\left(G_{\lambda}(b)\right)=\sum_{d \in \mathcal{D}_{\tilde{\mathcal{B}}[\lambda]}, b^{\prime} \in \mathcal{B}(\lambda)} c_{\beta_{b} d}^{\beta_{b^{\prime}}} b^{\prime}, \quad(b \in \mathcal{B}(\lambda)) .
$$

Then we have $\Psi\left(G_{\lambda}(b)\right)=\Phi\left(G\left(\beta_{b}\right)\right) u_{\lambda}$, since $t_{\beta^{\prime}} u_{\lambda}=b^{\prime}$ if $\beta^{\prime}=\beta_{b^{\prime}}$ and 0 otherwise by Theorem 6.44. We get

$$
\Psi\left(G(\beta) G_{\lambda}(b)\right)=\Phi\left(G(\beta) G\left(\beta_{b}\right)\right) u_{\lambda}=\Phi(G(\beta)) \Phi\left(G\left(\beta_{b}\right)\right) u_{\lambda}=\Phi(G(\beta)) \Psi\left(G_{\lambda}(b)\right) .
$$


This shows that $\Psi$ is $\widetilde{\mathbf{U}}$-linear.

Let $\mathcal{L}(\lambda)_{0}$ be the $\mathbf{A}_{0}$-submodule of $V(\lambda)_{0} \otimes_{\mathbf{Z}} \mathbf{Q}\left(q_{s}\right)$ generated by $q^{-a\left(\beta_{b}\right)} b(b \in$ $\mathcal{B}(\lambda))$. Note that $a\left(\beta_{b}\right)$ is independent of $b$ by Lemma $6.33\left(\right.$ ii). Since $q^{a\left(\beta_{b^{\prime}}\right)} c_{\beta_{b} d}^{\beta_{b^{\prime}}}=$ $q^{a\left(\beta_{b}\right)} c_{\beta_{b} d}^{\beta_{b^{\prime}}} \in \mathbf{Z}\left[q_{s}\right]$ by Lemma $6.33(\mathrm{i})$, we have $\Psi(\mathcal{L}(\lambda)) \subset \mathcal{L}(\lambda)_{0}$. The induced homomorphism $\Psi_{0}: \mathcal{L}(\lambda) / q_{s} \mathcal{L}(\lambda) \rightarrow \mathcal{L}(\lambda)_{0} / q_{s} \mathcal{L}(\lambda)_{0}$ is given by

$$
\Psi_{0}(b)=\sum_{d \in \mathcal{D}_{\tilde{\mathcal{B}}[\lambda]}, b^{\prime} \in \mathcal{B}(\lambda)} \gamma_{\beta_{b} d}^{\beta_{b^{\prime}} b^{\prime}}
$$

where $b^{\prime} \in \mathcal{B}(\lambda)$ is identified with $q^{-a\left(\beta_{b^{\prime}}\right)} b^{\prime} \bmod q_{s} \mathcal{L}(\lambda)_{0}$. By Theorem 6.44 we have $\gamma_{\beta_{b} d}^{\beta_{b^{\prime}}}=\delta_{b b^{\prime}} \delta_{\beta_{b}, \beta_{b} d}$, hence the right hand side is equal to $b$. This shows that $\Psi$ is an isomorphism as in the proof of Corollary 4.15.

Proposition 6.47. For $\widetilde{\mathbf{U}}\left(A_{n}^{(1)}\right)$ we have $\# \mathcal{D}_{\tilde{\mathcal{B}}[\lambda]}=\prod_{i=1}^{n}\left(\begin{array}{c}n+1 \\ i\end{array}\right)^{\lambda_{i}}$.

Proof. We check the dimension of $W(\lambda)=\bigotimes_{i} W\left(\varpi_{i}\right)^{\otimes \lambda_{i}}$. The result follows if we show the dimension of $W\left(\varpi_{i}\right)$ is $\left(\begin{array}{c}n+1 \\ i\end{array}\right)$. By considering the Drinfeld polynomials of $W\left(\varpi_{i}\right)$ [30, Remark 3.3], each is an evaluation module where the underlying finite dimensional representation of $\widetilde{\mathbf{U}}\left(A_{n}\right)$ is $V\left(\omega_{i}\right)$ where the $\omega_{i}$ are the fundamental weights of type $A_{n}$ [7, Prop. 12.2.13]. These are of dimension $\left(\begin{array}{c}n+1 \\ i\end{array}\right)$ for $i=1, \ldots, n$.

Remark 6.48. The above argument shows that the number of $\mathcal{D}_{\tilde{\mathcal{B}}[\lambda]}$ can be given if we know $\operatorname{dim} W\left(\varpi_{i}\right)$ for all $i \in I_{0}$. They are known for untwisted affine Lie algebras for classical groups and some exceptional groups [8]. The second author writes a computer program for them (for untwisted cases) by using Frenkel-Mukhin's algorithm [13]. The program gives us answers except one fundamental representation for $E_{8}$, corresponding to the triple node.

\section{REFERENCES}

[1] T. Akasaka, An integral PBW basis of the quantum affine algebra of type $A_{2}^{(2)}$, Publ. RIMS 38 (2002), 803-894.

[2] T. Akasaka and M. Kashiwara, Finite-dimensional representations of quantum affine algebras, Publ. RIMS 33 (1997), 839-867.

[3] J. Beck, Braid group action and quantum affine algebras, Comm. Math. Phys. 165 (1994), $555-568$.

[4] Convex bases of PBW type for quantum affine algebras, Comm. Math. Phys. 165 (1994), 193-199.

[5] - Crystal structure of level zero extremal weight modules, Lett. Math. Phys. 61 (2002), 221-229.

[6] J. Beck, V. Chari and A. Pressley, An algebraic characterization of the affine canonical basis, Duke Math. J. 99 (1999), 455-487.

[7] V. Chari and A. Pressley, A Guide to Quantum Groups, Cambridge University Press, 1994.

[8] _ Minimal affinizations of representations of quantum groups: the simply laced case, J. Algebra 184 (1996), 1-30.

[9] Q Quantum affine algebras at roots of unity, Representation Theory 1 (1997), 280-328.

[10] — Twisted quantum affine algebras, Comm. Math. Phys. 196 (1998), 461-476.

[11] I. Damiani, La R-matrice pour les algèbres quantiques de type affine non tordu, Ann. scient. Ec. Norm. Sup. 31 (1998), 493-523.

[12] - The R-matrix for (twisted) affine algebras, in Representations and quantizations (Shanghai, 1998), 89-144, China High. Educ. Press, Beijing, 2000.

[13] E. Frenkel and E. Mukhin, Combinatorics of q-characters of finite-dimensional representations of quantum affine algebras, Comm. Math. Phys. 216 (2001), 23-57. 
[14] H. Garland, The arithmetic theory of loop algebras, J. Algebra 53 (1978), no. 2, 480-551.

[15] V.G. Kac, Infinite dimensional Lie algebras (3rd Ed.), Cambridge University Press, 1990.

[16] M. Kashiwara, On crystal bases of the q-analogue of universal enveloping algebras, Duke Math. J. 63 (1991), 465-516.

[17] _ Crystal bases of modified quantized enveloping algebra, Duke Math. J. 73 (1994), 383-413.

[18] - On level zero representations of quantized enveloping algebras, Duke Math. J. 112 (2002), 117-175.

[19] S.Z. Levendorskii, Ya. S. Soibelman, Some applications of the quantum Weyl groups, J. Geom. Phys. 7 (1990), no. 2, 241-254.

[20] G. Lusztig, Cells in affine Weyl groups IV, J. Fac. Sci. Univ. Tokyo Sect. IA Math. 36 (1989), $297-328$.

[21] G. Lusztig, Canonical bases arising from quantized enveloping algebras, J. Amer. Math. Soc. 3 (1990), 447-498.

[22] _ Canonical bases and tensor products, Proc. of the Nat. Acad. Sci. USA 89 (1992), $8177-8179$.

[23] Introduction to Quantum Groups, Progress in Math. 110, Birkhäuser, 1993.

[24] Quantum groups at $v=\infty$, Functional Analysis on the eve of the 21st century, Vo. 1 (New Brunswick, NJ, 1993), 199-221, Progr. Math. 131, Birkhäuser, Boston.

[25] _ Braid group action and canonical bases, Adv. Math. 122 (1996), 237-261.

[26] I.G. Macdonald, Symmetric functions and Hall polynomials (2nd ed.), Oxford Math. Monographs, Oxford Univ. Press, 1995.

[27] K. McGerty, Cells in quantum affine $\mathfrak{s l}_{n}$, preprint, math.QA/0209055.

[28] D. Mitzman, Integral bases for affine Lie algebras and their universal enveloping algebras, Contemporary Mathematics, 40, American Mathematical Society, Providence, RI, 1985.

[29] H. Nakajima, Quiver varieties and finite dimensional representations of quantum affine algebras, J. Amer. Math. Soc. 14 (2001), 145-238.

[30] _ Extremal weight modules of quantum affine algebras, preprint, math.QA/0204183.

[31] T. Nakashima, Crystallized Peter-Weyl type decomposition for level 0 part of modified quantum algebra $\tilde{U}_{q}\left(\widehat{\mathfrak{s l}}_{2}\right)_{0}$, J. Algebra 189 (1997), 150-186.

[32] Y. Saito, PBW basis of quantized universal enveloping algebras, Publ. RIMS, 30 (1994), 209-232.

[33] M. Varagnolo and E. Vasserot, Canonical bases and quiver varieties, preprint, math.RT/ 0107177.

Department of Mathematics, Bar Ilan University, 52900 Ramat Gan, Israel

E-mail address: beck@macs.biu.ac.il

Department of Mathematics, Kyoto University, Kyoto 606-8502, Japan

E-mail address: nakajima@kusm.kyoto-u.ac.jp 